$8376^{3}$

\title{
Cumulative-Strain-Damage Model of Ductile Fracture: Simulation and Prediction of Engineering Fracture Tests
}

M. L. Wilkins

R. D. Streit

J. E. Reaugh

October 3,1980 


\section{DISCLAIMER}

This report was prepared as an account of work sponsored by an agency of the United States Government. Neither the United States Government nor any agency Thereof, nor any of their employees, makes any warranty, express or implied, or assumes any legal liability or responsibility for the accuracy, completeness, or usefulness of any information, apparatus, product, or process disclosed, or represents that its use would not infringe privately owned rights. Reference herein to any specific commercial product, process, or service by trade name, trademark, manufacturer, or otherwise does not necessarily constitute or imply its endorsement, recommendation, or favoring by the United States Government or any agency thereof. The views and opinions of authors expressed herein do not necessarily state or reflect those of the United States Government or any agency thereof. 


\section{DISCLAIMER}

Portions of this document may be illegible in electronic image products. Images are produced from the best available original document. 
UCRL-53058

Distribution Category UC-25

\title{
Cumulative-Strain-Damage Model of Ductile Fracture: Simulation and Prediction of Engineering Fracture Tests
}

\author{
M. L. Wilkins \\ R. D. Streit \\ J. E. Reaugh*
}

Manuscript date: October 3, 1980

This document is

PUBLICLY RELEASABLE

B Steule

Authorizing Official

Date: $\quad 9-12 \cdot 07$

*Science Applications, Inc.,

San Leandro, California 94577

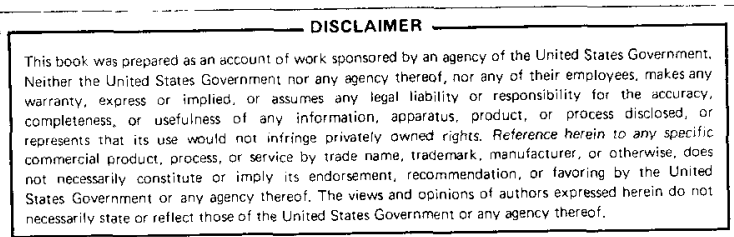

LAWRENCE LIVERMORE LABORATORY

University of California $\bullet$ Livermore, California $\bullet 94550$ 
Abstract

Introduction

Background

Cumulative-Damage Model

Fracture Mode 1

Strain Damage

Damage History

Material Size Effect

Calibration for 6061-T651 Aluminum

Implementation in Numerical Simulations of Fracture Application to Compact Tension Tests Conclusions

$$
\begin{aligned}
& \text { CTOD and } r_{c} \\
& J_{I C} \text { and } D_{c} \text {. }
\end{aligned}
$$

Acknowledgments

Appendix: Experimental Calibration and Verification of the Damage Model

A Method for Determining the Work Hardening

Function to Describe Plasticity of Metals

The Need for Constitutive Relations

Constitutive Relations

Tension Test

Impact Experiments

Torsion Tests

Plasticity Function for Combined Stress Loading

Tension Test of Flat Plates

Notched Cylinders

Conclusions

Calibration and Verification of Failure

Criteria in Ductile Fracture

Notched-Tension Tests

Torsion Tests

Tension-Torsion Tests

Conclusion 
CUMULATIVE-STRAIN-DAMAGE MODEL OF DUCTILE FRACTURE :

SIMULATION AND PREDICTION OF ENGINEERING FRACTURE TESTS

\section{ABSTRACT}

A cumulative-strain-damage criterion is used to predict the initiation and propagation of fracture in ductile materials. The model is consistent with a model of ductile rupture that involves void growth and coalescence. Two- and three-dimensional finite difference computer codes, which use incremental-plasticity theory to describe large strains with rotation, are used to trace the history of damage in a material due to external forces. Fracture begins when the damage exceeds a critical value over a critical distance and proceeds as the critical-damage state is reached elsewhere. This unified approach to failure prediction can be applied to an arbitrary geometry if the material behavior has been adequately characterized. The damage function must be calibrated for a particular material using various material property tests. The fracture toughness of 6061-T651 aluminum is predicted.

\section{INTRODUCTION}

Fracture of ductile materials in engineering structures can often be related to gross yielding, elastic-plastic crack growth, or the initiation and propagation of cracks in the linear elastic regime. Although the criteria are generally treated independently, they all involve material separations that are very similar on a microscopic scale. The distinction between the criteria appears to be due to the evolution of engineering design analysis; i.e., the use of tensile properties was developed long before the recognition of fracture mechanics as a design concept. Thus, while the material may not recognize these differences, it is useful as a design aid to treat these failures separately. A fracture model that is consistent with a void-growth-and-coalescence mechanism for ductile fracture should be capable of predicting fracture regardless of the loading or geometry of the structure involved. Such a model gives a unified picture of observed material. 
responses, from linear-elastic fracture to elastic-plastic or fully plastic behaviors.

\section{BACKGROUND}

Most engineering structure contain flaws or cracks. Thus engineering design often requires evaluation of the maximum flaw size and operating stress level for safe operation. Large flaws and/or high stresses can lead to crack growth and ultimately to unstable propagation and structural failure. Knowledge of the fracture toughness of a material, a measure of its resistance to crack growth, is required to design against unstable crack propagation.

Smal1-scale specimens can be used to determine the resistance of a material to crack propagation, but measurements taken at small scale do not necessarily coincide with large-scale results. Structures that are large enough fail by brittle fracture.* In the brittle-fracture regime, the failure stress varies inversely as the square root of the size, so that larger geometrically similar structures will fail by brittle fracture at a lower average stress. Smaller similar structures will fail at a higher average stress, until a certain size is reached. For further reduction in size the failure mode changes to ductile fracture. For small-scale testing to be successful, the size-effect scaling laws must be understood. 1

Linear elastic-fracture mechanics (LEFM) successfully describes the scaling law for brittle fracture. The material is treated as a linear elastic solid, and the stress field at a crack tip due to a remote stress is calculated. Figure 1 shows the well-known solution to this problem for positions near the tip of a crack. ${ }^{2}$

The stress field in Fig. 1 is characterized by the stress-intensity factor $\mathrm{K}_{\mathrm{I}}$. For certain geometries, the value of $\mathrm{K}_{\mathrm{I}}$ at the crack tip has

* Brittle fracture refers to plane strain fracture as predicted by linear elastic fracture mechanics. The micromechanism leading to fracture is assumed to be simple rupture due to microvoid coalescence whether the macro-scale is ductile or brittle. 


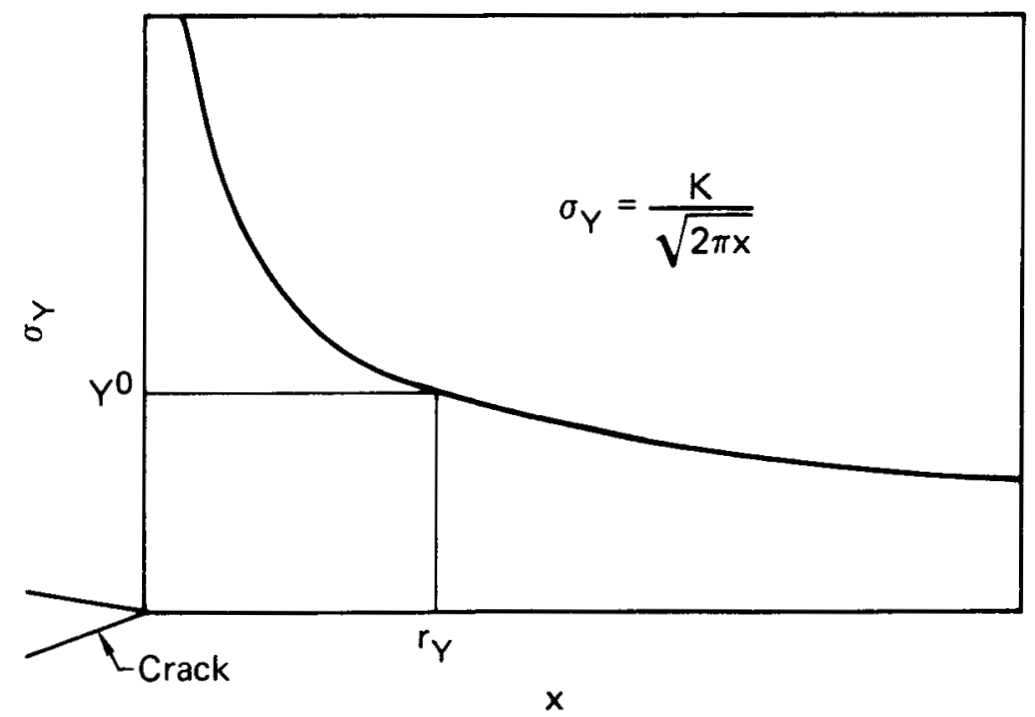

Fig. 1. Elastic stress field in the vicinity of a crack tip, arising from a remote load perpendicular to the crack.

been calculated in terms of the geometry and far-field stress. For example, the stress intensity factor for a crack of length 2 a in a plate of width w pulled in tension by a far-field stress $\sigma_{\infty}$ is ${ }^{3}$

$$
K_{I}=\sigma_{\infty} \sqrt{\pi a}\left(\frac{w}{\pi a} \tan \frac{\pi a}{w}\right)^{1 / 2}
$$

when $a / w$ is small,

$$
K_{I}=\sigma_{\infty} \sqrt{\pi a}
$$

Thus, the applied load and external geometry of the component are connected by linear elastic theory to a value of $K_{I}$ at the crack tip.

A reasonable postulate is that crack growth occurs when a critical stress $\sigma_{c}$ extends over a critical distance $r_{c}$." The value of the stress-intensity factor $K_{I}$ for crack extension under these conditions is called the plane strain fracture toughness $\mathrm{K}_{\mathrm{Ic}}$. Thus a single parameter $\mathrm{K}_{\mathrm{Ic}}$ describes fracture initiation where the existence of a critical stress $\sigma_{c}$ and a critical distance $r_{c}$ is assumed but the quantities are not evaluated. A mathematically equivalent postulate is that fracture occurs when the strain energy extends over a sufficient distance, i.e., when $\sigma^{2} r$ exceeds some limiting value. 
The presence of a crack with a stress concentration as shown in Fig. 1 can lead to catastrophic brittle fracture even when the average stress through a section is we 11 below the flow stress used in design of the structure. The advantages of the $K_{I c}$ concept for evaluating fracture toughness are: (1) An analysis can be made that relates external physical measurements on a test specimen to a reasonable postulate for fracture initiation; (2) the method is conservative, in that it evaluates the materiai resistance to fracture at worst-case conditions, i.e., those under which brittle fracture occurs.

A through-the-thickness crack in a piate and the compact tension specimen provide practical plane strain geometries that reproduce the stress conditions of Fig. l, provided that certain size requirements are met. The important factors in selecting the dimensions of the small-scale test specimen are the crack length and the specimen thickness. LEFM is postulated on elastic behavior. However, the very high local stress in front of the crack always leads to plastic flow at a crack tip. For the analysis to be valid, the extent of plastic flow must be small compared to the specimen dimensions and crack length. If the crack is too short compared to the remaining ligament,* gross yielding will occur before fracture conditions are reached, and some unknown combination of plastic flow and fracture properties is then being measured. If the crack is too long, the stress field at the crack tip can interact with the boundaries of the specimen. In either case, the analysis is incorrect and the $\mathrm{K}_{\mathrm{Ic}}$ test is not valid.

Figure 1 shows the position $r_{y}$ ahead of the crack tip that corresponds to $\sigma_{y}=Y^{0}$, where $Y^{0}$ is the flow stress. A valid $K_{I c}$ test requires that $r_{Y}$ be small compared to the crack length. The thickness dimension is important because the normal stress at a free surface is zero. If $\sigma_{z}$ is the stress in the thickness direction, then $\sigma_{z}=-P+s_{z z}=0$ at the lateral boundaries of the specimen, where $P$ is the hydrostatic pressure and $s_{z z}$ is a stress deviator. At the elastic limit, $s_{z z}$ is limited by the material flow stress. As a consequence, the hydrostatic pressure is limited at the lateral boundaries. As the load on the crack tip of the specimen is increased, the fact that the hydrostatic pressure is limited at the boundaries affects an increasingly larger portion of the load-bearing surface in the plane of the crack. The purpose of plane strain testing is to evaluate the resistance to fracture of a material under conditions in which the hydrostatic *The remaining ligament is the length of the segment in the crack plane that bears the applied load. 
tension is not limited. The specimen thickness must be chosen large enough that plane strain conditions exist when fracture first begins.

For the reasons given above, materials with high fracture resistance and/or low flow stress require large test specimens to achieve the desired fracture conditions. A convenient parameter for correlating dimensions of cracked bodies is

$$
\mathrm{L}_{\mathrm{Ic}}=\left(\frac{\mathrm{K}_{\mathrm{Ic}}}{\mathrm{Y}^{0}}\right)^{2}
$$

which has the dimensions of length. Irwin ${ }^{4}$ has pointed out the importance of this parameter. The value of $\mathrm{L}_{\mathrm{Ic}}$ determines whether the behavior is brittle or ductile. ${ }^{5}$ If the structure is small compared to $\mathrm{L}_{\mathrm{Ic}}$, the failure mode will be ductile; if the structure is large compared to $\mathrm{L}_{I_{c}}$, the failure mode will be brittle. Valid $K_{I c}$ tests require that the minimum thickness of a compact tension specimen be greater than $2.5 \mathrm{~L}$ Ic ${ }^{6}$ For lowand intermediate-strength steels, $L_{I c}$ ranges from 0.02 to $40 \mathrm{in} .^{5}$ LEFM is too conservative when the test specimen size requirement is larger than the actual structure; in this case the structure does not fail by brittle fracture. The problem cf structural design is to determine fracture resistance when both $L_{I_{c}}$ and the structure are large, and when it is impractical to test at the actual size of the structure.

The J integral, originally defined by Rice, ${ }^{7}$ is a path-independent energy line integral for two-dimensional problems. Using the "HRR" model developed by Hutchinson ${ }^{8}$ and by Rice and Rosengren ${ }^{9}$ to describe the stress-strain field in the vicinity of a crack, McClintock ${ }^{10}$ concluded that $J$ is a measure of the plastic stress and strain singularity near a crack $t i p$. With such an interpretation, we may regard the field-characterizing parameter $J$, for the plastic case, as analogous to the stress-intensity factor $K$ in LEFM. A description of $J$ as a fracture criterion is given in Refs. 11 and 12. As in the $K$ method, the applied load and the external geometry of the specimen are connected by theory to a value of $\mathrm{J}$ at the crack tip. A critical value for crack extension, J $\mathrm{Ic}_{\mathrm{c}}$, is identified. With the J method, the specimen thickness for a compact tension test must exceed $15 \mathrm{~J} / \mathrm{Y}^{0}$ (see Ref. 8). Since the $J$ integral accounts for plasticity, the method permits compact tension tests on specimens many times smaller than permitted by the $\mathrm{K}_{\text {Ic }}$ method. 
The $\mathrm{J}$ method is based on a one-parameter model of fracture. It overcomes a major limitation of the $K$ method by including stable crack growth with increased load. A limitation of the $\mathrm{J}$ method is that deformation plasticity is used to describe plastic flow. This non-physical model of plastic behavior can only provide an analysis for modest crack growth (growth of approximately $6 \%$ of the remaining 1 igament, according to Ref.10). Figure 2, taken from Ref. 14 , compares the $\mathrm{K}$ and $\mathrm{J}$ methods with experimental results for fracture propagation under plastic conditions. The crack growth is stable, since an increase in the applied stress is required to extend the crack after initiation at point $A$. The $K$ method incorrectly predicts $\sigma a^{l / 2}=$ constant. If $J_{\text {Ic }}$ is accepted as a fracture criterion, and if conditions for ductile behavior are met, larger flaws than would be permitted by the $\mathrm{K}_{\mathrm{Ic}}$ criterion can be tolerated for the safe operation of a structure.

Nevertheless, the $\mathrm{J}$ method must be applied conservatively, because of the limitations of the analysis and because of incomplete experimental data. As shown in Fig. 2, the $\mathrm{J}_{\mathrm{Ic}}$ criterion permits only slight crack extension, although considerable stable extension may in fact be possible. An analysis that could describe observed crack growth up to the limit load (point $B$ in Fig. 2) would safely extend the service life of the structure by delaying repair until a much larger crack developed.

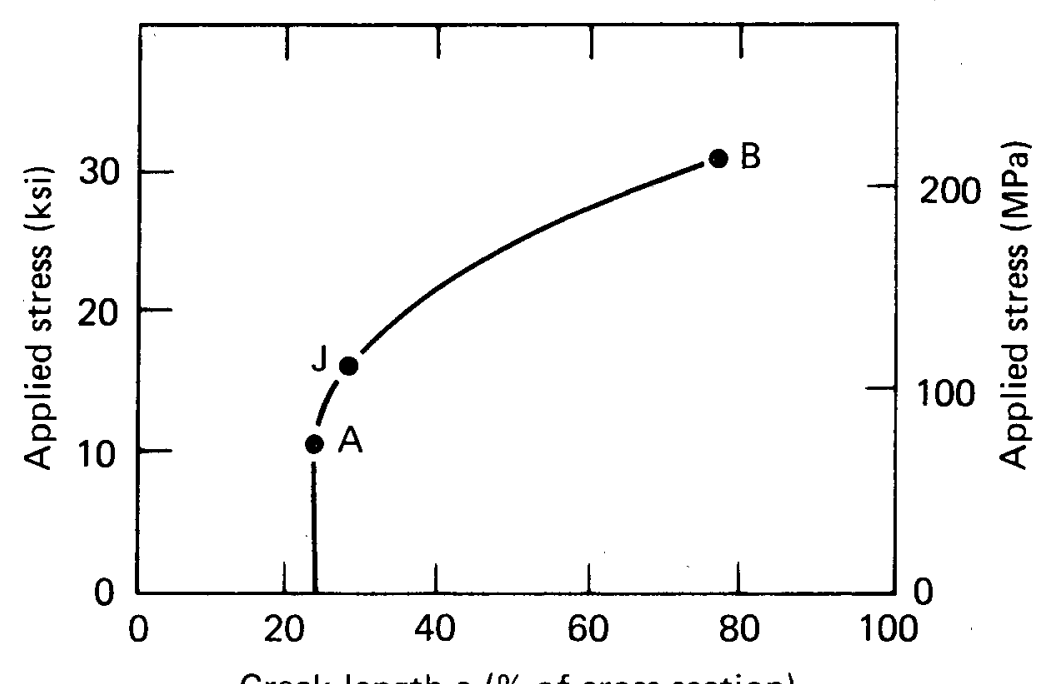

Crack length a (\% of cross section)

Fig. 2. Schematic representation of crack initiation and propagation for ductile material behavior. Initial crack length $25 \%$ of cross section. Line A-B: Experimental stable crack growth. Point A: Limit of $\mathrm{K}$-method analysis. Line A-J: Limit of J-method analysis. 
Plane-strain fracture-toughness testing, in which a crack is loaded in tension, attempts to evaluate fracture behavior when the hydrostatic tension is large compared to the flow stress. A fracture process that occurs at conditions of high hydrostatic tension without an initial crack is spallation resulting from the impact of two solids. Compressive stress waves generated at impact reflect from boundaries and produce tensile stresses within the solids, causing brittle fracture. There is a geometric size effect similar to that found in fracture-mechanics studies. In small-scale experiments, fracture requires larger tensile stresses, achieved by higher impact velocities, than required in geometrically similar large-scale experiments. Tuler and Butcher ${ }^{15}$ showed that spall experiments could be correlated by a cumulative damage parameter D: fracture occurs for

$$
D=\int\left(\sigma-\sigma^{0}\right)^{2} d t>D_{c r i t},
$$

i.e, when the tensile stress o exceeds a threshold stress $\sigma^{0}$ for a sufficient time.

A time-dependent material behavior is not necessarily implied by these experiments. Colliding-plate experiments can produce one-dimensional strain states that cannot be reached statically because of the motion of lateral boundaries in static experiments. The same correlation of experimental dynamic fracture data is obtained when the incremental time dt in Eq. (1) is replaced by an incremental distance dr divided by an arbitrary velocity. With this substitution, Eq. (1) takes the form $\sigma^{2} r=$ constant or $\sigma \mathrm{r}^{1 / 2}=$ constant. Thus, fracture from dynamic spall experiments correlates in the same manner as in static fracture experiments, and a time-dependent material behavior is not required to explain fracture by spallation. The spall results and the $\mathrm{K}_{\mathrm{Ic}}$ analysis have in common a one-parameter model that satisfactorily correlates experimental data for fracture at high hydrostatic tension. The parameters $K_{I c}$ and $D_{c r i t}$ are not material parameters. since they serve only to correlate fracture data at high hydrostatic tensions.

A size effect is revealed by the fact that small specimens sustain higher tensile stresses than do large geometrically similar specimens. We consider 
this to be due to a material property size effect, namely that stress or damage of sufficient magnitude must extend over a definite minimum distance before fracture will begin.

Below we describe a model for fracture initiation and propagation that is independent of specimen size and that describes the material behavior up to the limit of load-carrying ability. A microscopic distance is incorporated to account for the material size effect. Macroscopic size-effect phenomena result from the model.

FRACTURE MODEL

\section{STRAIN DAMAGE}

The cumulative-strain-damage model assumes that fracture is the result of the history of strain damage to the material. Two factors enhance strain damage: hydrostatic tension and asymmetric strain. A damage history based on these factors follows McClintock's theory of ductile fracture. 16

Hydrostatic tension accounts for the growth of holes in fracture by spalling, in which the loading consists of large triaxial stress and small strain. Interrupted tension tests reveal the initiation and growth of voids that link with neighbors to form fracture surfaces. Incipient-spall experiments show the same progression.

Asymmetric strain accounts for the observation that the elongation before failure decreases as the shear load increases in fracture tests with combinedstress loads. This was noted by Mogi, 17 who studied the effect of intermediate stress on the fracture of rocks. Mogi's data show that elongation decreases when tensile fracture occurs with confining stresses that produce asymmetric strains. We assume that, after initiation, the holes can link up as a band if subsequent loading is shear.

\section{DAMAGE HISTORY}

The simplest expression for the damage $D$ that follows the concepts just described is 
$D=\int w_{1} w_{2} d \bar{\varepsilon}^{p}$,

where

$\bar{\varepsilon}^{P}=$ equivalent plastic strain,

$w_{1}=$ hydrostatic-pressure weighting term $=\left(\frac{1}{1+a P}\right)^{\alpha}$,

$w_{2}=$ asymmetric-strain weighting term $=(2-A)^{B}$,

$A=\operatorname{Max}\left(\frac{s_{2}}{s_{3}}, \frac{s_{2}}{s_{1}}\right), \quad s_{1}>s_{2}>s_{3}$.

Here $P$ is the hydrostatic pressure; $s_{1}, s_{2}$, and $s_{3}$ are the principal stress deviators; and $a, \alpha$, and $B$ are material constants. The parameter $A$ ranges from 0 to 1 ; we call the stress field symmetric when $A=1$ and asymetric when $A=0$. These limits correspond to the loading conditions for the simple tension test and the torsion test, respectively.

\section{MATERIAL SIZE EFFECT}

Fracture begins when the cumulative damage $D$ exceeds a critical damage $D_{c}$ over a critical distance $r_{c} ; D_{c}$ and $r_{c}$ are considered material constants.

CALIBRATION FOR 6061-T651 ALUMINUM

The damage model was calibrated for 6061-T651 aluminum by simulating simple tension tests and fracture experiments on notched cylinders, flat plates, and notched plates, and analyzing the resulting stress- and strain-field histories up to the initiation of fracture. In a11, 14 geometries were examined. These tests are described in detail in the Appendix. The best fit to the damage model is given by the parameters listed in Table 1 . 
TABLE 1. Parameters for damage model fit to 6061-T651 aluminum.

Fracture Model

Parameters

Equation of State

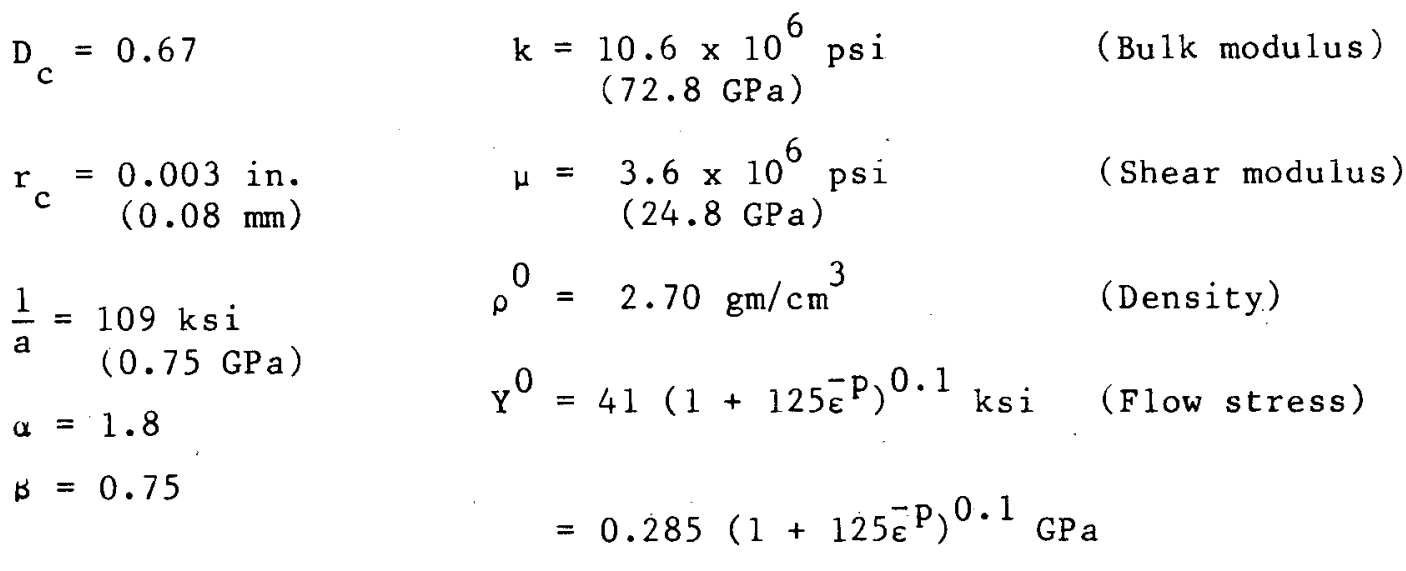

I MPLEMENTATION IN NUMERICAL SIMULATIONS OF FRACTURE

Numerical simulations of fracture, whether by the finite-difference methods used here or by finite-element methods, have a characteristic dimension, the zone or element size, that must be considered when implementing a damage model of fracture that itself includes a material length $r_{c} \cdot$ If the zone size is smaller than $r_{c}$, the damage $D$ centered in the zone that breaks first must exceed $D_{c}$ by an amount that depends on the local damage gradient, the zone size, and $r_{c}$. If the zone size is twice $r_{c}$, $D$ must equal $D_{c}$ since the damage is defined at the zone center. If the zone size exceeds $r_{c}$, but is smaller than the distance to the elastic-plastic boundary in the direction of crack advance (Fig. 1), fracture begins when D is smaller than $D_{c}$ by an amount that again depends on the local damage gradient, the zone size, and $r_{c}$.

In certain cases, implementation of the model can be simplified by using. our knowledge of the plastic-strain and damage fields near notches and cracks. Simulations of material tests using notched tension specimens show the plastic-strain field just before fracture begins has the form

$$
\bar{\varepsilon}^{\mathrm{P}}=\bar{\varepsilon}_{\mathrm{N}}^{\mathrm{P}} \exp \left(-\mathrm{r} / \mathrm{r}_{0}\right)
$$


where $\bar{\varepsilon}_{\mathrm{N}}^{\mathrm{p}}$ is the (extrapolated) strain at the notch surface, $\mathrm{r}_{0}$ is roughly equal to the notch-root radius, and $r$ is the distance below the notch. This exponential dependence appears to hold for sharp cracks also, over a distance ahead of the crack tip approximately equal to the crack-tip opening displacement $\delta$. For sharp cracks, $r_{0} \simeq \delta / 2$. For positions between $o$ and $r y$, the strain field at fracture initiation is approximately as predicted by $\mathrm{J}$ theory,

$$
\bar{\varepsilon}^{\mathrm{P}} \propto \mathrm{x}^{-1}
$$

where $x$ is the distance in front of the crack tip as in Fig. 1.

For 6061-T651 aluminum, $r_{c} \simeq 2 \delta$ at the point of initiation of sharp cracks. For simulations of fracture advance in precracked 6061-T651 specimens, the zone-centered damage given by

$$
D_{F} \simeq \frac{2 D_{c}{ }^{r} c}{\Delta x}
$$

must be reached, where $\Delta x$ is the zone size in the direction of fracture advance and $\Delta x / 2$ is the distance from the zone center to the crack tip. This simplification is only valid for fracture advance from a sharp crack, and it assumes a particular functional form for the damage field near an advancing crack tip, i.e., $D x=$ const.

\section{APPLICATION TO COMPACT TENSION TESTS}

Figure 3 shows the geometry used in tests to check the damage model. The specimen thicknesses of $0.5 \mathrm{in.}(12.7 \mathrm{~mm})$ and $0.15 \mathrm{in} .(3.81 \mathrm{~mm})$ meet the $\mathrm{J}$-integral criterion on $\mathrm{J}_{\mathrm{Ic}}$, since they exceed $25 \mathrm{~J}_{\mathrm{Ic}} / \mathrm{Y}^{0}$. (From the work of Begley et al., ${ }^{18} \mathrm{~J}_{\mathrm{Ic}}=80 \mathrm{in.} \cdot 1 \mathrm{~b} / \mathrm{in} .^{2}(0.014 \mathrm{MN} / \mathrm{m})$ and $\mathrm{Y}^{0}=41 \mathrm{ksi}(283 \mathrm{MPa}):$ Thus the thickness must exceed $0.05 \mathrm{in} .(1.27 \mathrm{~mm})$.) The other dimensions of the specimen follow the standard geometry for the 1/2-T compact tension test. All dimensions of the compact specimen can be referred to the thickness as a geometric scale factor. (The designation 1/2-T 


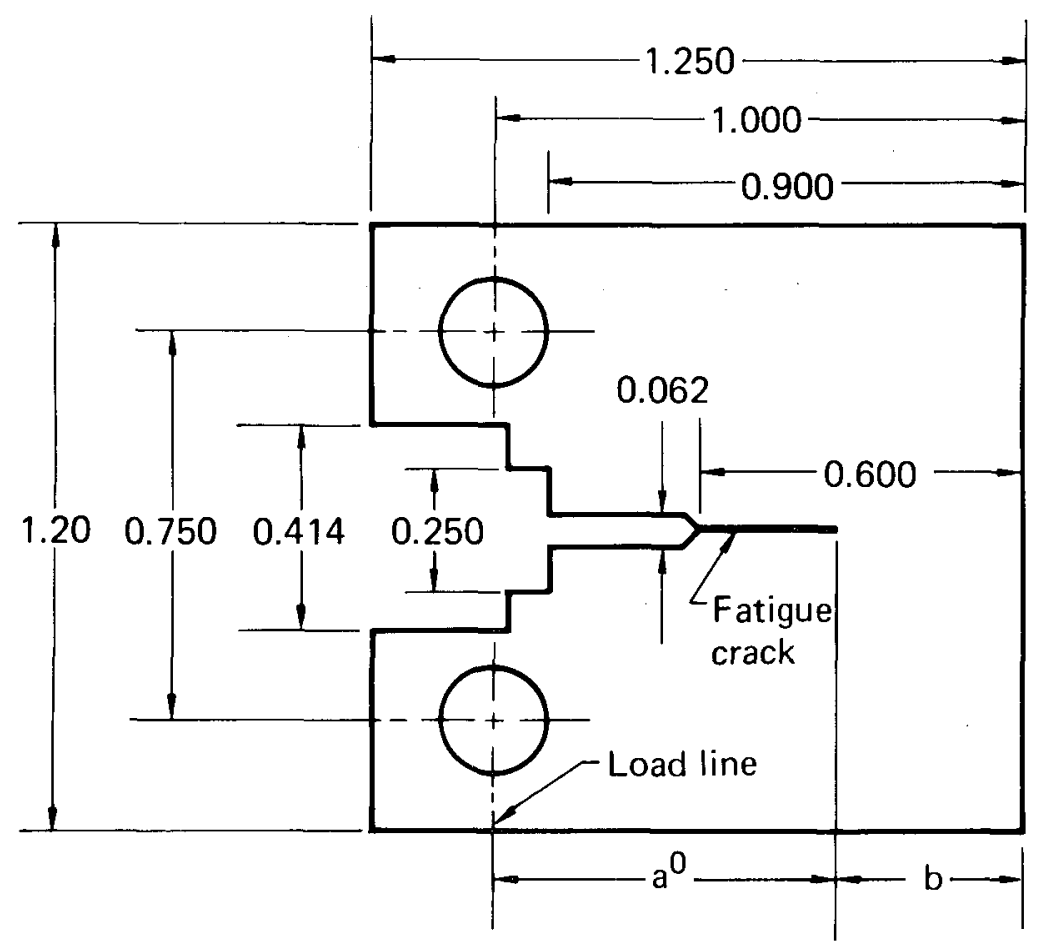

\begin{tabular}{|c|c|}
\hline$T A B$ & Thickness \\
\hline 01 & 0.150 \\
\hline 02 & 0.500 \\
\hline
\end{tabular}

Fig. 3. Compact tension test on 6061-T651 aluminum. Distance $a^{0}$ is from end of fatigue crack to load line; $b$ is length of uncracked ligament. All dimensions in inches.

is used for 1/2-in.-thick specimens.) We followed the standard ASTM J-integral experimental technique, in which a plot of load vs load-line displacement is made for several nominally identical specimens. Each sample was loaded to a different displacement and after unloading was examined for crack extension. A value of $\mathrm{J}$ was calculated for each specimen from the relation $J=(A / B b) f(a / w)$, where $A$ is the area under the load displacement curve, $B$ is the specimen thickness, and $b$ is the length of the uncracked ligament. The value of $f(a / w)$ was essentially constant at 2.2 for the specimens investigated. Figure 4 shows the experimental results.

The calculational model for the two geometries is shown in Fig. 5. The calculation was done with the time-dependent finite-difference program HEMP 3-D. 19 The specimen was loaded by applying a small velocity at the load-pin position. The displacement was recorded for a position corresponding to the 


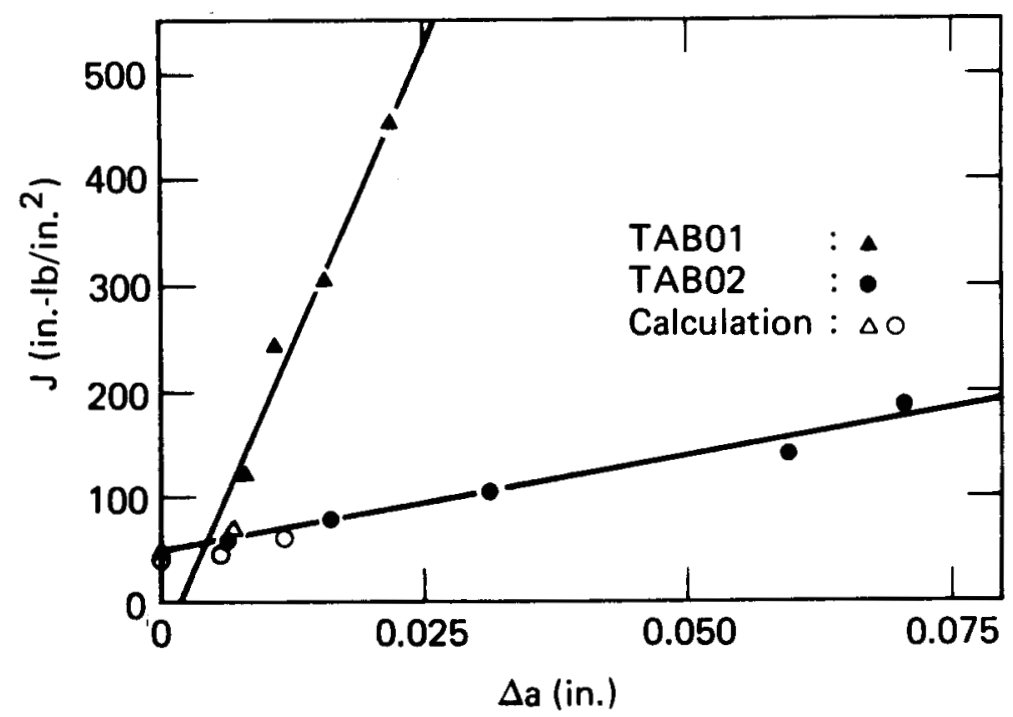

Fig. 4. J vs crack extension $\Delta$ a for 6061-T651 a luminum. Initial crack length $a^{0}=0.614$ in., uncracked ligament $b=0.382$ in. Specimen dimensions given in Fig. 3 .

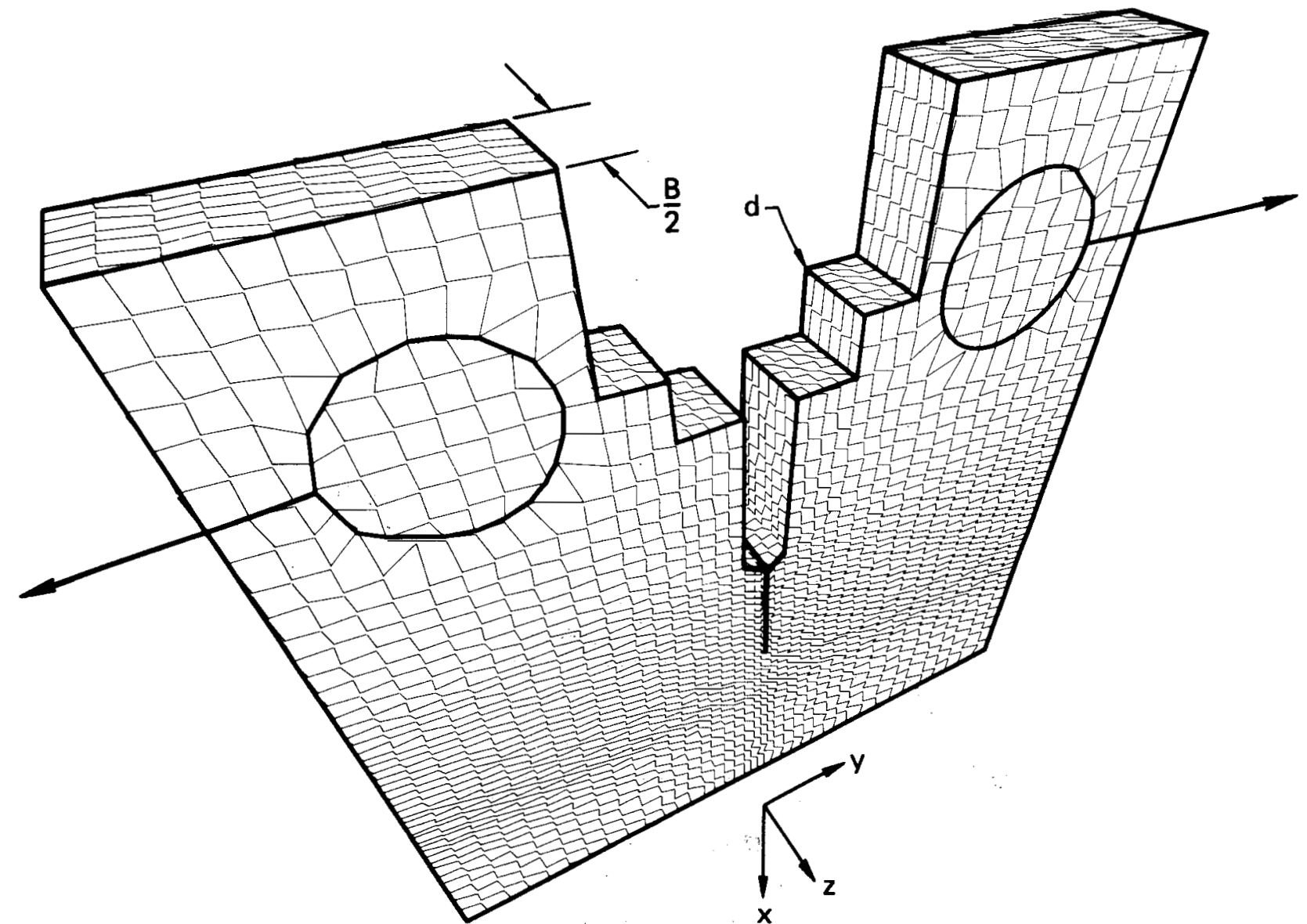

Fig. 5. Computer model for simulation of experiment $T A B 01, B / 2=0.075$ in.; 6 zones. The rear surface is a plane of symmetry. TAB 02 is the same, but $B / 2=0.25$ in.; 16 zones. Arrows show direction of pull. The load line displacement is recorded at point $d$. 
clip gauge of the experiment, and the load was calculated by integrating the calculated stress field over the ligament area.

Figure 6 shows the calculated load per unit area vs displacement. Fracture began in both specimens at an average stress well below the flow stress. In the thick specimens, fracture began while the average stress-strain behavior was still. linear, while in the thin specimens gross yielding occurred before fracture began.

Figure 7 shows the calculated stress profile along the thick-specimen 1 igament centerline just before critical damage was reached. The stress is primarily hydrostatic tension for about half the ligament, and is then compressive until the opposite surface is reached. Figure 8 shows the accumulated load across the ligament surface starting from the crack tip. The total load on the specimen is carried on the first 0.015 in. of the ligament (measured from the crack tip); i.e., the load on the remainder of the ligament balances to zero. The result that high loads are supported in very small distances ahead of the crack has a strong influence on the choice of near-tip zone size and zone-release mechanism.

Fracture propagation was simulated by applying free-surface conditions to a node point that had reached the failure condition. The kinetic energy introduced by the method of loading the specimen in the computer simulation did not affect fracture initiation. However, the method of simulating

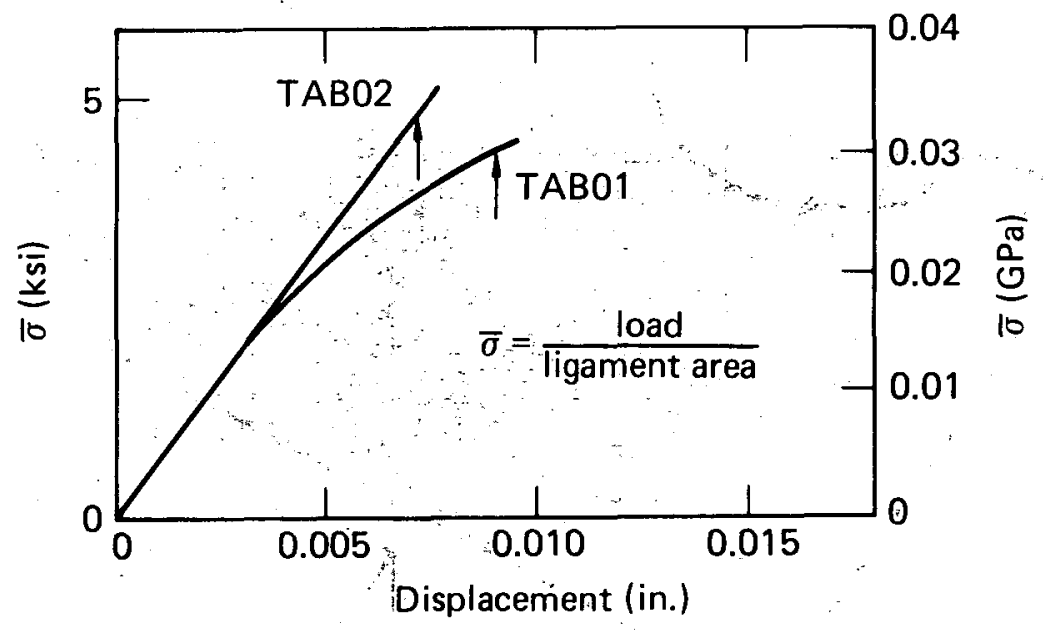

Fig. 6. Calculated average stress $\bar{\sigma}$ vs displacement (at point d, Fig. 5); arrows indicate fracture initiation. $\mathrm{TAB} 01$ Ligament area $=0.382 \times 0.15=0.0573 \mathrm{in.} 2$

$\mathrm{TAB} 02$ Ligament area $=0.382 \times 0.5=0.191$ in. 2 


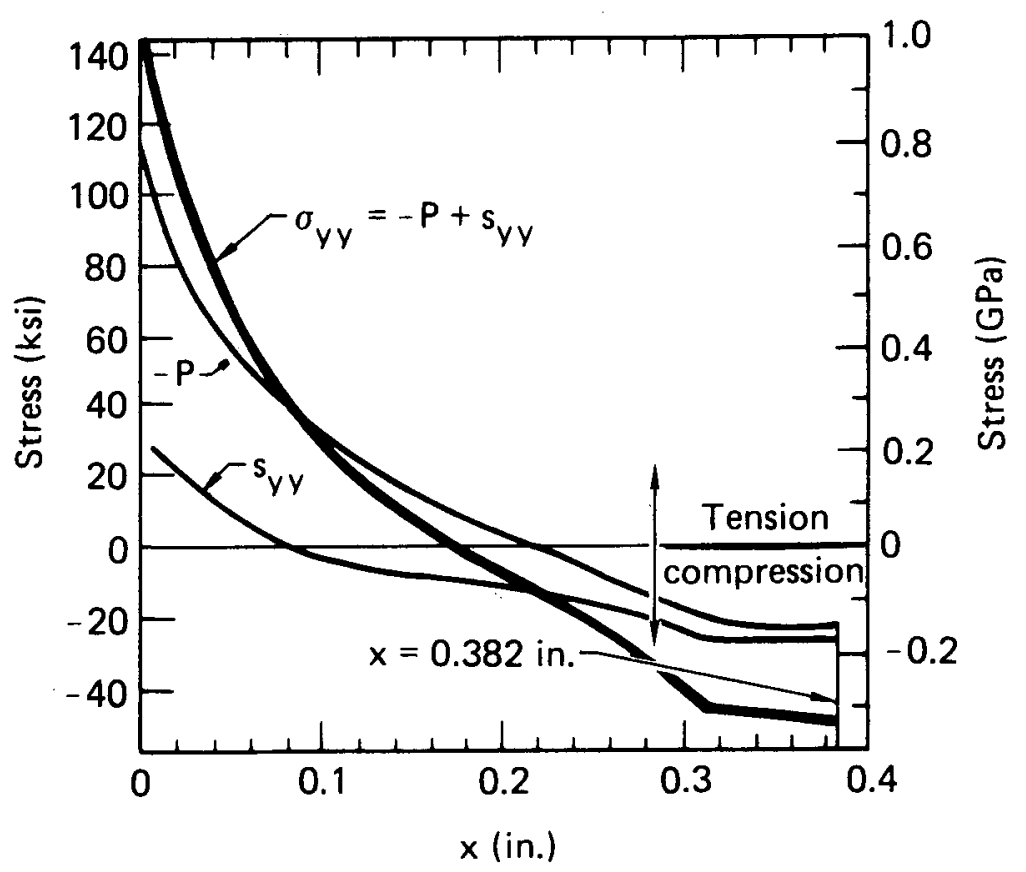

Fig. $7(a)$. Calculated stress profiles at ligament center for TAB 02 at fracture initiation. The quantity $x$ is the distance from the fatigue crack tip.

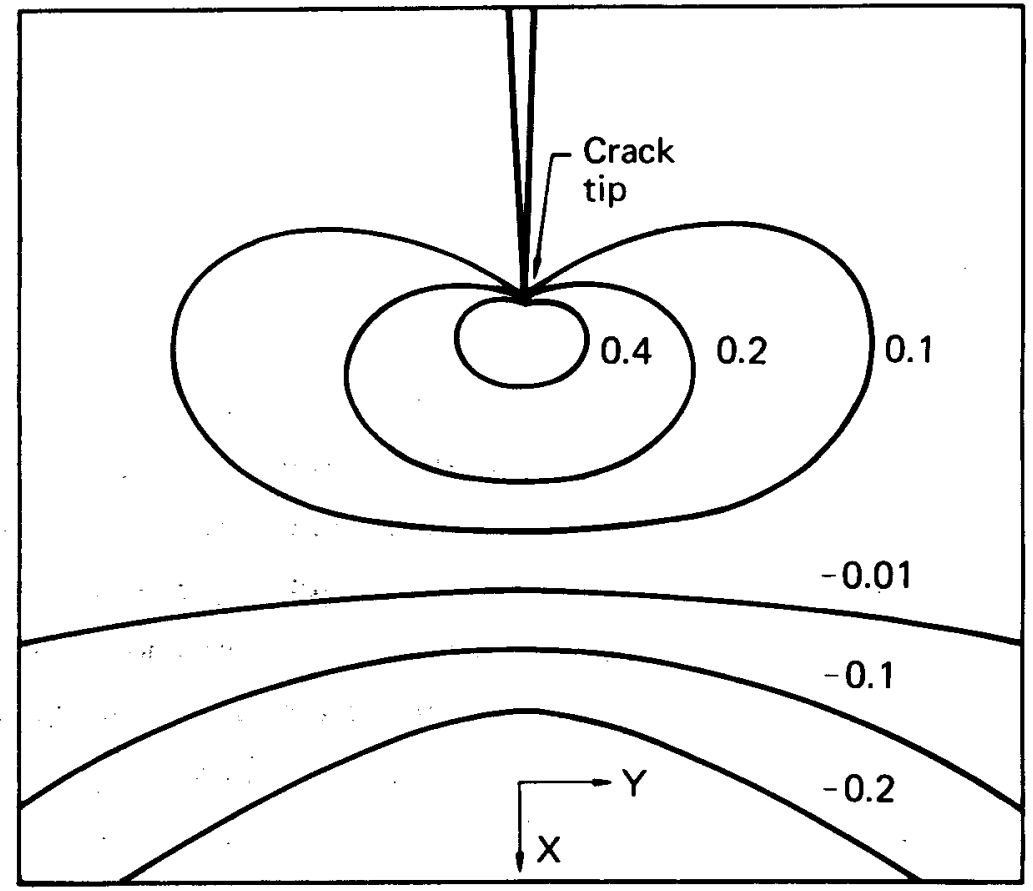

Fig. $7(b)$. Calculated contours of constant $\sigma_{y y}$, in $\mathrm{GPa}$, under conditions of $\mathrm{Fig}(7 \mathrm{a})$. 


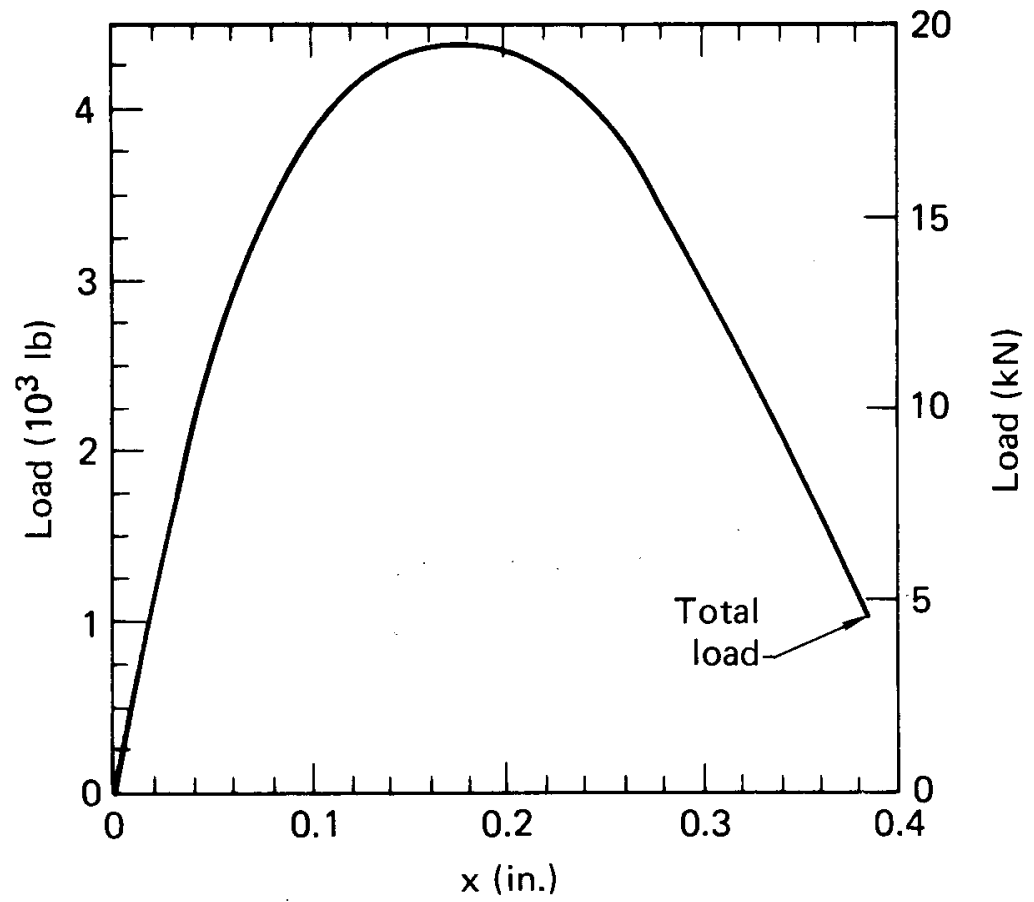

Fig. 8. Load along ligament for TAB 02. The quantity $x$ is the distance from the crack tip.

propagation did produce a dynamic effect. When the free-surface boundary conditions were applied to a node that met the failure conditions, the kinetic energy released drove the damage function of the adjacent nodes. The amount of energy released can be appreciated by reference to Fig. 8, which shows that the creation of a small surface area can release a very large fraction of the total load on the ligament. A computational technique was developed to damp the kinetic energy that results when the free surface conditions are applied, and this allowed us to approach a quasi-static result.

The usual analysis of experimental data in the J-integral technique takes $J_{\text {Ic }}$ to be the intersection of a graph of $J$ vs crack extension $\Delta a$ with a "blunting line." It is assumed that the crack extends some distance without creating a new surface. The calculations show this does not happen; the crack tip moves in the direction that shortens the crack before fracture occurs. Apparently, the experimentally observed crack extension on the blunting line is, in fact, the growth of a zone of intense plastic stretch on the crack surface that begins at the tip and moves back along the flank of the crack. This stretch is developed as the crack profile changes to the characteristically blunt notch shape before the crack extends. Since this stretch zone is not directly related to crack growth, and since, in our 
calculations, there is no clear-cut demarcation of the stretch zone, we take $J_{\text {Ic }}$ to correspond to the intersection of the J-vs- $\Delta$ a curve with the $J$ axis, as in Fig. 4. Since the blunting line for 6061-T651 aluminum is much steeper than $J$ vs $\Delta a$, results from either method are essentially the same. (For materials that exhibit large changes in $J$ with $\Delta a$, our model would suggest that J ${ }_{I c}$ should be taken where $\Delta a=r_{c} \cdot$ ) The experimental results for the thick specimens give $J=5 I \mathrm{in. \bullet 1b/in.}{ }^{2}$ The same analysis applied to the thin specimen yields a negative value for $\mathrm{J}$.

The calculated HEMP 3D results are shown in Fig. 4. Taking the J corresponding to the first initiation or "pop-in" as $\mathrm{J}_{\mathrm{Ic}}$, we obtain $\mathrm{J}_{\mathrm{Ic}}=40 \mathrm{in} \cdot \cdot \mathrm{lb} / \mathrm{in}^{2}(0.07 \mathrm{MN} / \mathrm{m})$ and $\mathrm{J}_{\mathrm{Ic}}=49 \mathrm{in} \cdot \cdot 1 \mathrm{~b} / \mathrm{in} .^{2}(0.086$ $\mathrm{MN} / \mathrm{m}$ ) for the $0.5-\mathrm{in}$. and $0.15-\mathrm{in}$. specimens respectively. Figure 4 shows that the calculated crack advance agrees with each experiment. The crack extension for the specimens proceeded by tunneling. A single value of $\Delta a$ was obtained by averaging through the thickness as described in the ASTM J procedure. The damage model reproduces the experimental data for each experiment even though the J-integral method did not give results of any significance for the thin specimen.

\section{CONCLUSIONS}

Our simple model describes fracture initiation and propagation. The mode 1 is consistent with the main features of ductile fracture by void initiation, growth, and coalescence, and contains a parameter $\left(r_{c}\right)$ that represents a microscopic or material size effect. The observed macroscopic size effect (brittle or ductile behavior) is a direct consequence of the model. The results predicted by the damage model agree with experimental data and, in particular, predict the necessary scaling relation for ductile fracture.

The excellent agreement of our model with experimental: data for fracture propagation was not a foregone conclusion. The model parameters were determined using data from fracture-initiation experiments; the fracture propagation results of the model were predictions. It is therefore reasonable to infer that the model represents the important phenomena of ductile fracture initiation and propagation. Since $J$ theory augmented by a measured increase in $\mathrm{J}$ with crack extension, also seems to describe fracture initiation and 
propagation, there should be a simple connection between our model and $\mathrm{J}$. We have not found such a simple connection, but we indicate here the 1 inks that have been uncovered.

CTOD AND $r_{c}$

For 6061-T651 aluminum, $r_{c}$ is approximately twice the crack-tip opening displacement (CTOD) at fracture initiation. A previous study of A533B stee $1^{20}$ showed that $r_{c}$ was approximately equal to CTOD for a $1-T$ compact specimen, which was marginally big enough to be a valid test. For two additional heat treatments of that steel, $r_{c}$ was twice CTOD and 2.5 times CTOD. Thus we tentatively assume that the $r_{c}$ of our model, which is used for both initiation and propagation, is approximately twice the CTOD of a compact tension specimen at initiation. We note further that $\mathrm{J}_{\mathrm{Ic}}$ should also, in theory, be related to CTOD by the approximate formula

$$
\text { CTOD }=\frac{2 \mathrm{~J}_{\mathrm{Ic}}}{\mathrm{Y}^{0}} .
$$

$\mathrm{J}_{\mathrm{Ic}}$ AND D

There is no convenient theoretical connection between $\mathrm{D}_{\mathrm{c}}$ and $\mathrm{J}_{\mathrm{Ic}}$. Although mathematicai expressions (Eqs. (8), (9), and (16)) have been given for both the stress field and the equivalent strain field as functions of $J$ and the work hardening behavior, these formulas are in serious error for plastic strain. (They are quite accurate for stress, however.) This has been shown by several authors, including McMeeking and Parks, ${ }^{21}$ by comparison of the analytical predictions with results from numerical simulations. Thus, at present, three experimental geometries are required to obtain parameters for our model: the tension test, to obtain the work-hardening behavior and plastic strain at fracture in the presence of intermediate hydrostatic tension; a notched tension test to obtain the strain at fracture with intermediate hydrostatic tension and intermediate asymmetric loading; and a fatigue-cracked compact test to obtain the parameter $r_{c}$ and the plastic strain at fracture with high hydrostatic tension. 
Two specimens are required for $\mathrm{J}_{\mathrm{Ic}}$ testing--the tension specimen and a compact specimen. However, the size of compact specimen required to obtain $\mathrm{r}_{c}$ is considerably smaller than that required for $\mathrm{J}_{\mathrm{Ic}}{ }$.

The value of our model is that fracture extension can be predicted beyond maximum load, without the size requirements of J testing. Thus we see our model, with detailed numerical simulations, as a means of extending the scope of fracture toughness measurement and its use in engineering design. In situations in which LEFM is valid, $\mathrm{K}_{\mathrm{Ic}}$ is appropriate for design. In situations in which $J$ theory is valid, $J_{I_{c}}$ and $J$ vs $\Delta a$ are appropriate for design. There are, however, situations that involve substantial plasticity and substantial stable crack growth. It is in those situations that our model, unlike either LEFM or $J$ theory, is valid and appropriate for both design and analysis of existing structures.

\section{ACKNOWLEDGMENTS}

This work was performed under the auspices of the U.S. Department of Energy by Lawrence Livermore National Laboratory under contract No. W-7405-Eng-48. The work was sponsored by the Electric Power Research Institute, Palo Alto, CA, under contract No. RP 603-1-7, 2 .

We thank R. Wullaert for conducting the compact-tension test and R. Gulliford for the calculations on HEMP 3D. 


\section{APPENDIX}

EXPERIMENTAL CALIBRATION AND VERIFICATION OF THE DAMAGE MODEL

The ability to predict failure of a component under arbitrary loading requires an accurate assessment of material behavior under a well-prescribed load. Such a "material model calibration" is often used to evaluate a material's yield strength, ultimate strength, and fracture resistance. The damage model requires a similar calibration. To calibrate the damage model, we use tests that characterize the material flow properties--both axial and shear--and the stress-strain state at incipient failure. The materials used in the current assessment are 6061-T6 and 6061-T651 aluminum. Ali 6061-T651 specimens were machined from a single piece to ensure property control. This material was attractive because of its availability, cost, ease of machining, and uniformity of microstructure. Further, the relatively low ultimate strength of 6061-T65l aluminum allowed us to investigate a wide range of specimen configurations. Ail tests were run at room temperature.

The standard tension specimen was used to establish the plastic flow relation employed in the computer calculation. This test also provided data on fracture initiation. Tests were also designed to study fracture initiation for different combinations of plastic strain, stress, and stress gradients. Axial (simple tension) and shear (torsion) behavior were investigated as was the effect of multiaxial stress states with enhanced hydrostatic tension (notched tension).

The combination of hydrostatic tension and shear can be related to the mechanisms of ductile fracture; i.e., hydrostatic stresses are associated with void growth, while the linking-up of voids can be attributed to shear deformation. Hence the order of the applied loads--i.e., hydrostatic followed by shear or vice versa--should be important in failure modeling.

An evaluation of the stress-order effect using tension/torsion samples was undertaken. Shear deformation with rotation (torsion) was also compared to shear in an axially loaded flat plate (limited rotation). The study of the effects of stress gradient employed the notched-tension and center-notched-panel tests. These tests were used to assess the characteristic size requirement necessary for fracture initiation. The 
following sections give a brief description of the various tests used to calibrate the damage model and verify its applicability.

The next section describes the methods used to evaluate the material constitutive equations. Tests used in this task included the simple tension, torsion, flat-plate tension, and notched-tension specimen. The section following that describes the tests used in the damage model calibration. These tests include: the tensile test of a plate with a central notch, and the notched-tension, torsion, and tension-torsion tests.

\section{A METHOD FOR DETERMINING THE WORK HARDENING \\ FUNCTION TO DESCRIBE PLASTICITY OF METALS}

\section{The Need for Constitutive Relations}

A constitutive equation is a model to describe physical behavior. Constitutive equations that describe the principal features of physical phenomena provide models of material behavior that permit calculations of a physical event to be carried to completion. For example, in the study of spall it is important to know the shape of a stress wave induced in a material in order to calculate the states of stress and strain when the wave interacts with a free boundary. A constitutive relation that adequately describes the evolution of a stress wave propagating through a given material is thus important in the spall problem, even though details of microscopic phenomena responsible for the wave shape may be unknown.

A constitutive equation is a model based on judgment. It is especially useful when it provides a wide-range description of the physical behavior. Three fundamental types of mechanical behavior can be associated with real materials: elasticity, plasticity, and possibly rate effects including viscosity. In the engineering application of metals to structural design, elastic-plastic properties are the main concern.

Elastic-plastic theory has been very successful in describing the behavior of metals and provides a good framework on which to build models of material behavior. In elastic-plastic theory, the total stress is composed of a hydrostatic component $\mathrm{P}$ and a deviatoric component $\mathrm{s}$; a 1 imit is imposed on the magnitude of the deviatoric stress by the yield condition. Constitutive relations for elastic-plastic theory are given by the following equations. 
Stress (Hooke's law). The stress relations follow Hooke's law, as described by
a) $\dot{s}_{i j}=2 \mu \dot{\varepsilon}_{i j}+\dot{\delta}_{i j}$,
b) $\quad \dot{\sigma}_{i j}=-\dot{p}+\dot{s}_{i j}$, for $i=j$,
$j=1,2,3$,
c) $\dot{\sigma}_{i j}=s_{i j} \quad$ for $i \neq j$,
d) $-\dot{\mathrm{P}}=\mathrm{K} \dot{\mathrm{V}} / \mathrm{V}$.

Here $\dot{\varepsilon}_{i j}$ is the strain rate deviator, $V$ is the volume, $\dot{\mu}$ is the shear modulus, $K$ is the bulk modulus, and $\delta_{i j}$ is a correction term for rigid rotation. The dot over a parameter means a time derivative along a particle path. The time derivative provides a desired ordered sequence for the incremental stress/strain relation, and no rate-dependent behavior is meant. Generally, either the von Mises or the Tresca yield criterion is used to predict yielding. The von Mises criterion is well suited for computer modeling and is discussed below.

von Mises Yield Condition. The von Mises model postulates that yielding will occur when the equivalent stress exceeds the material flow stress as defined in uniaxial tension, i.e.,

$$
\begin{aligned}
& \sigma_{\text {eq }}-Y \geq 0, \\
& \sigma=\text { equivalent stress }=\sqrt{\frac{3}{2}} \sqrt{2 \mathrm{~J}}, \\
& Y=\text { flow stress }=H\left(\bar{\varepsilon}^{P}\right) .
\end{aligned}
$$

Here $2 \mathrm{~J}$ is the second invariant of the deviatoric stress tensor. The total strains are assumed to be the sum of elastic and plastic components. It is 
implicit in this formulation that the sum of the plastic strains is zero (plastic incompressibility). The equivalent plastic strain $\bar{\varepsilon}^{\mathrm{p}}$ is obtalned by integration during plastic deformation:

$$
\bar{\varepsilon}^{\mathrm{p}}=\int \mathrm{d} \bar{\varepsilon}^{\mathrm{p}},
$$

where

$$
d \bar{\varepsilon} P=\sqrt{\frac{2}{3} d^{P} \varepsilon_{i j} d^{P} \varepsilon_{i j}}
$$

the ${ }^{P_{\varepsilon}}{ }_{i j}$ are components of the plastic strain.

In satisfying the von Mises condition, the plastic strain-rate vector associated with a principal stress vector must be directed outwards along the normal to the yield surface. Computer simulation programs that incorporate the above model of elastic-plastic flow are described in Refs. 22 and 23.

The shear modulus $\mu$ and bulk modulus $k$ can be obtained from ultrasonic measurements. Knowledge of the work-hardening function $H\left(\bar{\varepsilon}^{\mathrm{p}}\right)$ is necessary for solutions of problems in which plastic deformation occurs.

\section{Tension Test}

Tne simple tension test of a cylindrical specimen offers a direct method for relating the equivalent stress $\sigma_{\text {eq }}$ to the equivalent plastic strain $\bar{\varepsilon}^{\mathrm{P}}$. For this test the equivalent stress coincides with the uniaxial stress $\sigma_{z z}$, and the equivalent strain coincides with the extension in the pulling direction. Very large local plastic strains can be produced when a ductile cylinder is pulled in tension. A slight taper is used in the cylindrical specimen to control the position of the large strains; the smallest diameter is at the midsection. The geometry permits very easy measurements of the equivalent stress and equivalent strain.

Stresses. The uniaxial stress is taken as the load divided by the midsection area and is usually called the true stress $\sigma_{T}$. Thus the proposition is

$$
\sigma_{z z}=\sigma_{\mathrm{T}}=\sigma_{\mathrm{eq}} .
$$


This is only strictly true before the elastic limit has been reached and while the axial stress $\sigma_{z z}$-is uniform across a section of the cylinder. As the plastic strain increases, $\sigma_{z z}$ and $\sigma_{e q}$ become increasingly nonuniform.

It is instructive to review the definitions of the axial and equivalent stresses. For the geometry of the tension test, the midsection is a plane of symmetry and the coordinate axes are the principal axes. The second invariant of the deviatoric stress tensor $2 \mathrm{~J}$ can be evaluated from the principal deviatoric stresses:

$$
2 J=s_{z z}^{2}+s_{r r}^{2}+s_{\theta \theta}^{2}
$$

The radial and hoop strains are the same, so $s_{r r}=s_{\theta \theta}$. Since $s_{z z}+s_{r r}+s_{\theta \theta}=0$, we have $s_{z z}=-2 s_{r r}$. Thus $2 J=3 s_{z z}^{2} / 2$, and

$$
\sigma_{\text {eq }}=\sqrt{\frac{3}{2}} \sqrt{2 J}=\frac{3 s z}{2} \text {. }
$$

The uniaxial stress is $\sigma_{z z}=-P+s_{z z}$. The radial stress $\sigma_{r r}$ must be zero at the cylinder free surface, i.e., $\sigma_{\mathrm{rr}}=-\mathrm{P}+\mathrm{s}_{\mathrm{rr}}=0$. Thus

$$
P=s_{r r}=-\frac{1}{2} s_{z z}
$$

and $\sigma_{z z}=-P+s_{z z}=3 s_{z z} / 2=\sigma_{e q}$. Of course this is not a result; rather, it is the basis for the original proposition.

After the elastic limit is reached and the tension load on the cylinder continues, the stresses depart from a uniform distribution. This is the region of interest, in which plastic flow occurs. The analysis of the experimental results of a tension test assumes that the average uniaxial stress $\bar{\sigma}_{z z}$, and the average equivalent stress $\bar{\sigma}_{\mathrm{eq}}$ are equal to the true stress $\sigma_{\mathrm{T}}$ :

$$
\bar{\sigma}_{z z}=\frac{2}{\pi R^{2}} \int_{r=0}^{r=R} \sigma_{z z}(r) r d r=\bar{\sigma}_{e q}=\frac{2}{\pi R^{2}} \int_{r=0}^{r=R} \sigma_{e q}(r) r d r=\sigma_{T}=\frac{l o a d}{\pi R^{2}} .
$$


Here $R$ is the current outside radius of the cylinder.

The elastic limit is reached first at the midsection, where the cross sectional area $\pi R^{2}$ is smallest and where the stresses are therefore largest. The flow stress $\mathrm{Y}$ at the midsection increases when plastic flow occurs. Positions adjacent to the midsection reach the elastic limit, and the process continues until plastic flow extends throughout the specimen length.

Strains. The magnitude of the plastic strain in the axial direction falls off as the axial distance from the midsection increases. A strain measurement must be taken over a region in which the strain is constant if the results are to be independent of the gauge length. In the tension test, the radial strain remains fairly constant even for large plastic deformations. The radial strajn can be obtained by measuring the change in diameter of the midsection; thus the diameter of the cylinder serves as the gauge length. We wish to obtain the strain in the axial direction--specifically, the equivalent plastic strain $\bar{\varepsilon}^{p}$. The simple analysis that follows shows the relationship between external measurements and strains in a cylinder.

External measurements of the radius $R$ and of an arbitrary axial length $L$, taken at the mid-plane of a cylinder, can be used to determine the average natural strains:

$$
\begin{array}{ll}
\varepsilon_{z z}=\int_{L}^{L} \frac{\mathrm{d} L}{\mathrm{~L}}=\ln \frac{\mathrm{L}}{\mathrm{L}^{0}} & \text { (axial strain) } \\
\varepsilon_{\mathrm{rr}}=\int_{\mathrm{R}^{0}}^{\mathrm{R}} \frac{\mathrm{dR}}{\mathrm{R}}=\ln \frac{\mathrm{R}}{\mathrm{R}^{0}} \quad \text { (radial strain) } \\
e_{\theta \theta}=\int \frac{\mathrm{dR} \mathrm{d} \theta}{\mathrm{R} d \theta}=\int_{\mathrm{R}}^{\mathrm{R}} \frac{\mathrm{dR}}{\mathrm{R}}=\ln \frac{\mathrm{R}}{\mathrm{R}^{0}} \quad \text { (hoop strain). }
\end{array}
$$

Here $L^{0}$ and $R^{0}$ are initial dimensions.

The strain $\varepsilon_{\theta \theta}$ is the result of the change in length of a linear element in the $\theta$ direction, where the change in length is due to a displacement in the r direction. The concept of natural strain compares the extension of an element of length to the current length rather than to the initial length. 
The volumetric strain $\int \mathrm{dv} / \mathrm{v}$ is

$$
\int \frac{\mathrm{dv}}{\mathrm{v}}=\int \frac{\mathrm{dL}}{\mathrm{L}}+2 \int \frac{\mathrm{dR}}{\mathrm{R}}
$$

hence

$$
\frac{\mathrm{v}}{\mathrm{v}^{0}}=\frac{\mathrm{L}}{\mathrm{L}^{0}}\left(\frac{\mathrm{R}}{\mathrm{R}^{0}}\right)^{2},
$$

where $\mathrm{v}^{0}$ is the initial volume.

The strains $\varepsilon_{z z}, \varepsilon_{r r}$, and $\varepsilon_{\theta \theta}$ include both elastic ( ${ }_{\varepsilon}$ )

and plastic $\left(P_{\varepsilon}\right)$ components, i.e., $\varepsilon_{z z}=e_{\varepsilon_{z z}}+P_{\varepsilon z z}$,

etc. The elastic components are small compared to the plastic components.

It is experimentally observed that the volume does not change during plastic flow (plastic incompressibility). Thus if $\mathrm{P}_{\varepsilon z}, \mathrm{P}_{\varepsilon r r}$, and $\mathrm{P}_{\varepsilon_{\theta \theta}}$ are the plastic strains,

$$
P_{\varepsilon_{z z}}+P_{\varepsilon r r}+P_{\varepsilon_{\theta \theta}}=0
$$

Here the plastic strains $P_{\varepsilon z}, P_{\varepsilon_{r r}}$, and $P_{\varepsilon_{\theta \theta}}$ are also the principal plastic strains. The equivalent plastic strain $\bar{\varepsilon}^{\mathrm{P}}$ for this case is given by

$$
\bar{\varepsilon}^{P}=\sqrt{\frac{2}{3}} \sqrt{\left(P_{\varepsilon_{z z}}-P_{\varepsilon_{r r}}\right)^{2}+\left(P_{\varepsilon_{r r}}-P_{\varepsilon_{\theta \theta}}\right)^{2}+\left(P_{\varepsilon_{\theta \theta}}-P_{\varepsilon_{z z}}\right)^{2}}
$$

with $\mathrm{P}_{\varepsilon_{z z}}=-2^{\mathrm{P}_{\varepsilon}}{ }_{\mathrm{rr}}=-{ }^{\mathrm{P}_{\varepsilon}}{ }_{r r}$, the equivalent plastic strain is the axial plastic strain: i.e., $\bar{\varepsilon}^{\mathrm{P}} \stackrel{\mathrm{rr}}{=} \mathrm{P}_{\varepsilon z z^{*}}$.

Experimental Results: 6061-T6 Aluminum. Figure A.1 gives the experimental results for two 6061-T6 aluminum tensile specimens. The same data are shown as both load and true stress $\sigma_{\mathrm{T}}$ vs radial strain $\varepsilon_{\mathrm{rr}^{*}}$ When the data are plotted as $\ln \sigma_{\mathrm{T}}$ vs $\ln \varepsilon_{\mathrm{rr}}$, a straight line is obtained. Thus the data fit the form $\sigma_{T}=a\left(\varepsilon_{z z}\right)^{n}$, where $\varepsilon_{z z}=-2 \varepsilon_{r r}=-2 \ln \mathrm{D} / \mathrm{D}^{0}$ and $D$ is the cylinder diameter. A convenient form in which to express the flow stress is $Y=a\left(b+\bar{\varepsilon}^{P}\right)^{n}$. Here $Y$ has replaced $\sigma_{T}$ and $\left(b+\bar{\varepsilon}^{P}\right)$ 

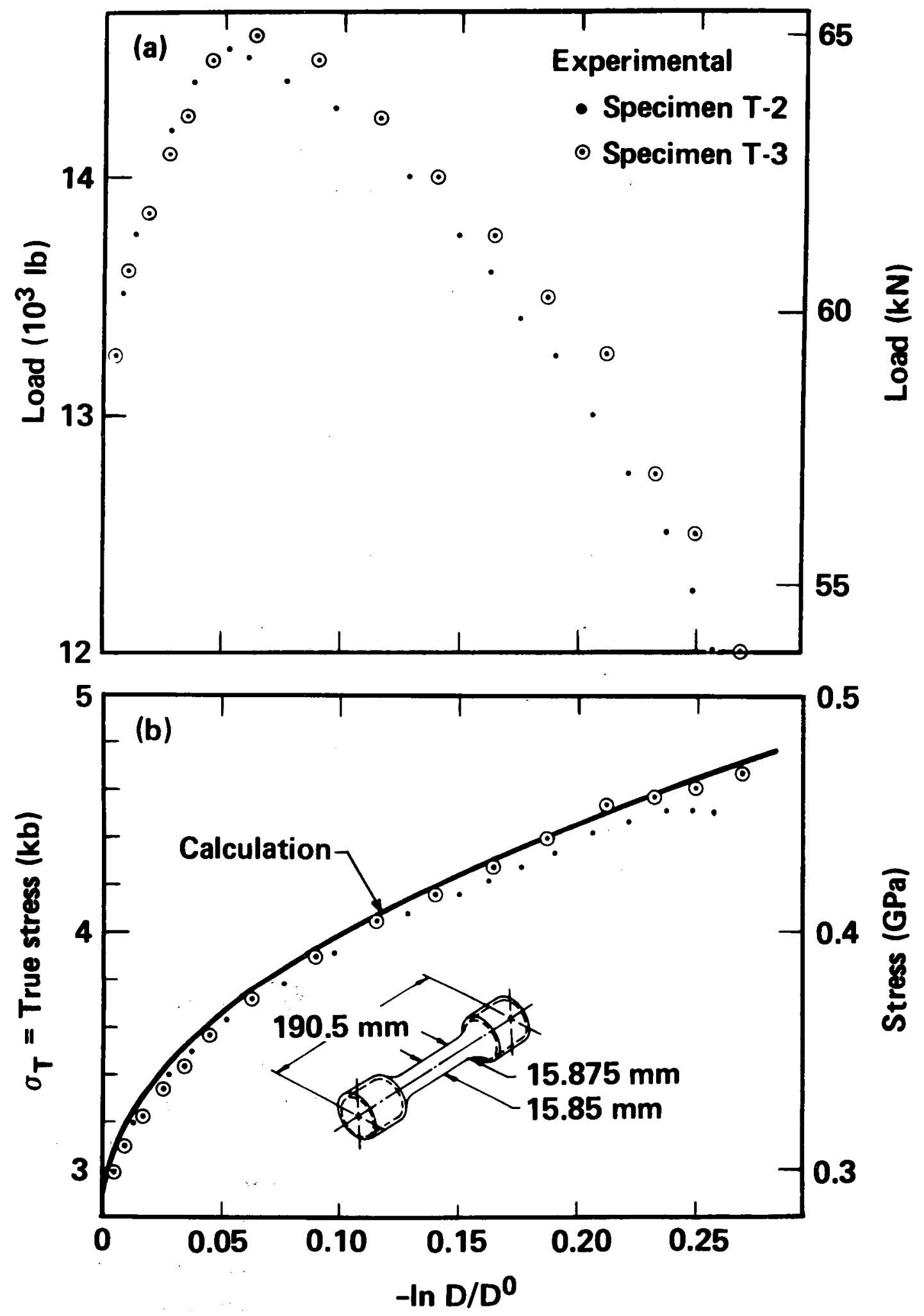

Fig. A.1. (a) Load and (b) true stress vs strain at midsection of a cylinder pulled in tension, original diameter $\mathrm{D}^{0}=15.85 \mathrm{~mm}$. Inset: dimensions of cylinder. 
has replaced $\varepsilon_{z z}$ in the relation $\sigma_{T}=a\left(\varepsilon_{z z}\right)^{n}$. The assumption here is that the average stress-strain data obtained experimentally can be used to suggest a relation for a point-function flow stress. The parameter $b$ could be considered to be the elastic strain, since the experimental strain $\varepsilon_{z z}=-2 \ln \left(D / D^{0}\right)$ includes the elastic strain. The elastic strain, of course, will change as the load changes. Since the components of elastic strains are small compared to the plastic strains, we will ignore them and consider $b$ as an empirical constant of the order of the strain at the elastic limit. It can easily be shown that, with this form for the flow stress, the exponent $\mathrm{n}$ corresponds to the axial strain at maximum load. Figure A.1 shows that this occurs at a radial strain of -0.05 , or an axial strain of 0.1 . It is of course important that fracture has not occurred in the region of the experimental data used to develop the plasticity function. Fracture of these tensile specimens originates at the center of the specimen. Examination of interrupted tests establishes that fracture begins after the peak load, when the radial strain is approximately 0.26 .

From the curve of load vs radial strain, the elastic limit is estimated to be $41 \mathrm{ksi}(284 \mathrm{MPa})$. This is consistent with Hugoniot elastic-1imit results that measure the elastic limit in compression. With the constants a and $b$ adjusted to give the flow stress at the elastic limit with $n=0.1$, the tension test can be simulated with the HEMP computer program. The resulting flow stress can be described by

$$
\mathrm{Y}=41\left(1+125 \bar{\varepsilon}^{\mathrm{P}}\right)^{0.1} \mathrm{ksi} \text {, or } \mathrm{Y}=284\left(1+125 \varepsilon^{-\mathrm{P}}\right)^{0.1} \mathrm{MPa} \text {. }
$$

Experimental Results: 6061-T651 Aluminum. Figure A.2 shows experimental results for tensile specimens machined from 6061-T65l aluminum. All tests were run in stroke control to avoid specimen instability at maximum load. The diametrical strain measurements were made during the test using photographic techniques with correlation to the load-stroke plot.

The test results show that all specimens behaved similarly (within experimental uncertainty) up to maximum load. This occurred at a radial strain of $-0.05 \pm 0.005$, or equivalently, an axial strain of $0.1 \pm 0.01$. However, at strains beyond this critical point, the necked cross section became more and more elliptical. All photographs were taken from one location, so we were unable to determine how this eccentricity progressed as 


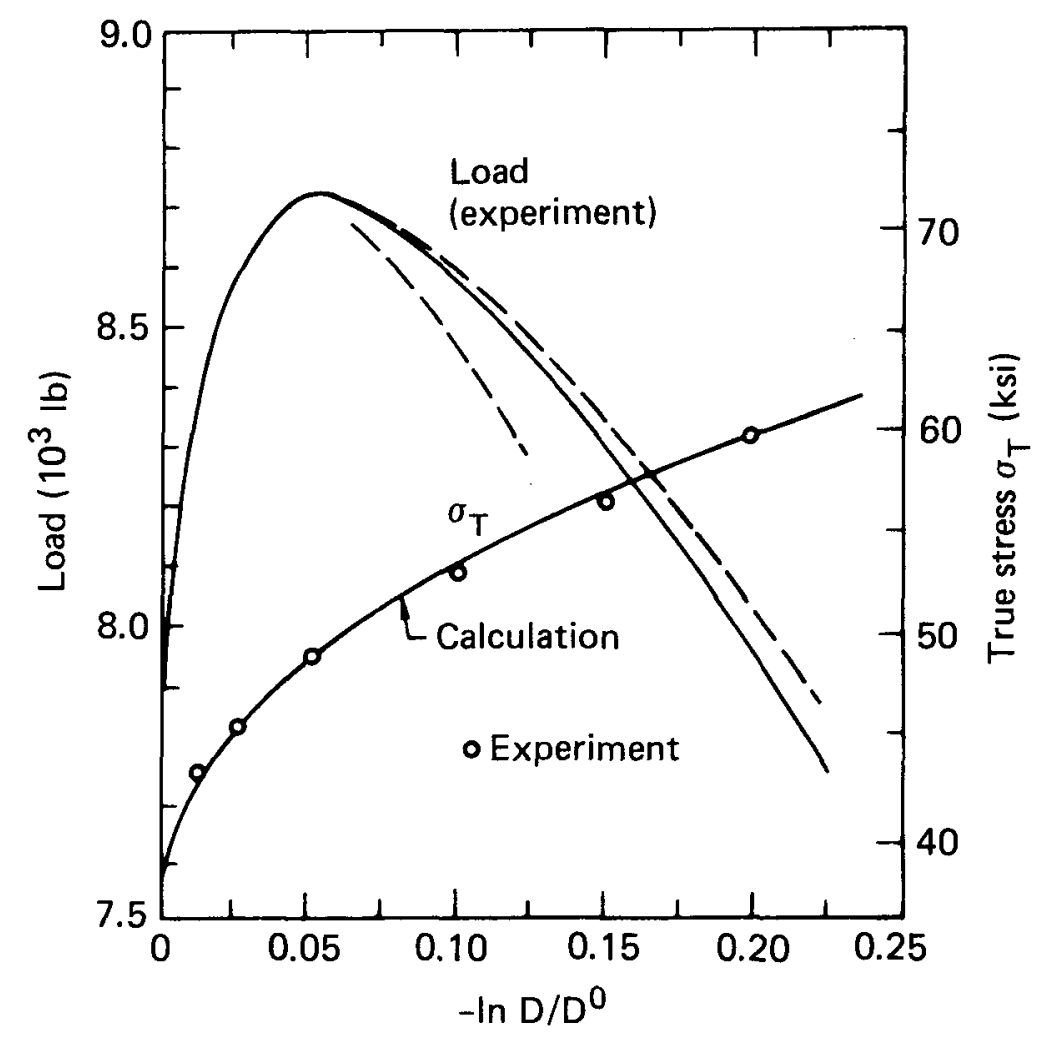

Fig. A.2. Load and true stress-vs-strain at the midsection of a 6061-T651 aluminum round bar pulled in tension. Original diameter $\mathrm{D}^{0}=12.7 \mathrm{~mm}$.

the strain increased. Post-failure inspection indicated that the final fracture area (necked area at failure) was consistent from specimen to specimen, with a reduction in area ( $R$. A.) of $67 \%$. The final eccentricity, $\mathrm{D}_{\max } / \mathrm{D}_{\min }$, was $1.12 \pm 0.02$ for all specimens. The final radial strain at fracture, calculated using an average neck diameter (determined from the neck area) resulted in $-\ln \left(D_{a v g} / D_{0}\right) \simeq 0.20$, so that $\left(\bar{\varepsilon}^{P}\right)$ avg $=0.40$. This is substantially. less than values obtained for 6061-T6 aluminum. However, the difference $c a n$ be reconciled if instead of using the average diameter, we incorporate the maximum strain or minimum diameter. With this diametrical measurement, the final plastic strain is $\left(\bar{\varepsilon}^{\mathrm{p}}\right)_{\max } \simeq 0.52$ $\left(-\ln \left(\mathrm{D} / \mathrm{D}_{0}\right)=0.26\right)$, in closer agreement with data on 6061-T6 aluminum. The eiliptical failure is believed to be a result of the processing of the 4-in. 6061-T65:1 plate used for the tests.

The experiment provides the true stress $\varepsilon_{T}$ as the load divided by the current cross sectional area at the midplane (as given in Fig. A.2). The axial stress $\sigma_{z z}$ and the true stress $\sigma_{T}$ are the same for this geometry until enough plastic strain has occurred and the stress profiles across the 
cylinder radius are no longer flat. The experimental data for true stress vs radial strain are used to estimate the dependence of the flow stress $Y$ on the equivalent plastic strain. Comparison with experiment is made with the computer simulation by suming the product of axial stress and elemental area for each computed point on the radius, and dividing by the cross sectional area. Fig. A.2 shows results assuming the flow stress of 6061-T651 aluminum is described by

$\mathrm{Y}=38.4\left(1+125 \bar{\varepsilon}^{\mathrm{p}}\right)^{0.1} \mathrm{ksi}$, or equivalently $265\left(1+125 \bar{\varepsilon}^{\mathrm{p}}\right)^{0.1} \mathrm{MPa}$. The study on 6061-T6 described earlier gave $Y=41\left(1+125 \bar{\varepsilon}^{\mathrm{p}}\right)^{0.1} \mathrm{ksi}$, or $284\left(1+125 \bar{\varepsilon}^{\mathrm{p}}\right)^{0.1} \mathrm{MPa}$.

Calculations. The HEMP finite difference code, ${ }^{22}$ used to simulate the tension test, solves the equations of continuum mechanics in two spatial dimensions and time. At each grid point, the incremental stress is obtained from strain increments that in turn are obtained from gradients of the velocity field. The constitutive model described earlier was used with the bulk modulus $K=10.6 \times 10^{6} \mathrm{psi}(72.8 \mathrm{GPa})$, the shear modulus $\mu=4.02 \times 10^{6}$ psi $(27.7 \mathrm{GPa})$, and the density $\rho^{0}=2.703 \mathrm{gm} / \mathrm{cm}^{3}$. The initial Lagrange grid used square zones, with 20 zones in the radial direction. A fixed axial velocity was applied to the cylinder end. Symmetry boundary conditions were applied at the position corresponding to the midsection of the experimental cylinder, which had an $0.5 \%$ taper. The program solves the constitutive equations at each zone at discrete time steps. The components of plastic strain are obtained by subtracting the elastic strains, obtained from the stress deviators, from the total strains. This procedure implicitly introduces plastic incompressibility into the model. The equivalent plastic strain $\bar{\varepsilon}^{\mathrm{P}}$ is evaluated at each zone, and is used to calculate the flow stress $Y=a\left(b+\bar{\varepsilon}^{p}\right)^{n}$ for the zone. Thus, as mentioned earlier, the average stress-strain behavior of the cylinder, obtained from external measurements, is used to suggest a point-function flow stress.

Figure A.1 compares the HEMP calculation with the experiment on 6061-T6 a luminum using the flow stress relation $Y=a\left(b+\bar{\varepsilon}^{P}\right)^{n}$, where $a=4.6$ kbar, $\mathrm{b}=0.008$, and $\mathrm{n}=0.1$; these values give $\mathrm{Y}=41\left(1+125 \bar{\varepsilon}^{\mathrm{P}}\right)^{0.1} \mathrm{ksi}$, or $\mathrm{Y}=284\left(1+125 \bar{\varepsilon}^{\mathrm{P}}\right)^{0.1} \mathrm{MPa}$. The calculated true stress shown in Fig. A. 1 is the average axial stress $\sigma_{z z}$ described earlier.

Figures A.3, A.4, and A.5 show calculated profiles at the midsection of the specimen when $R / R^{0}=0.772$, the value just before fracture. The stress 


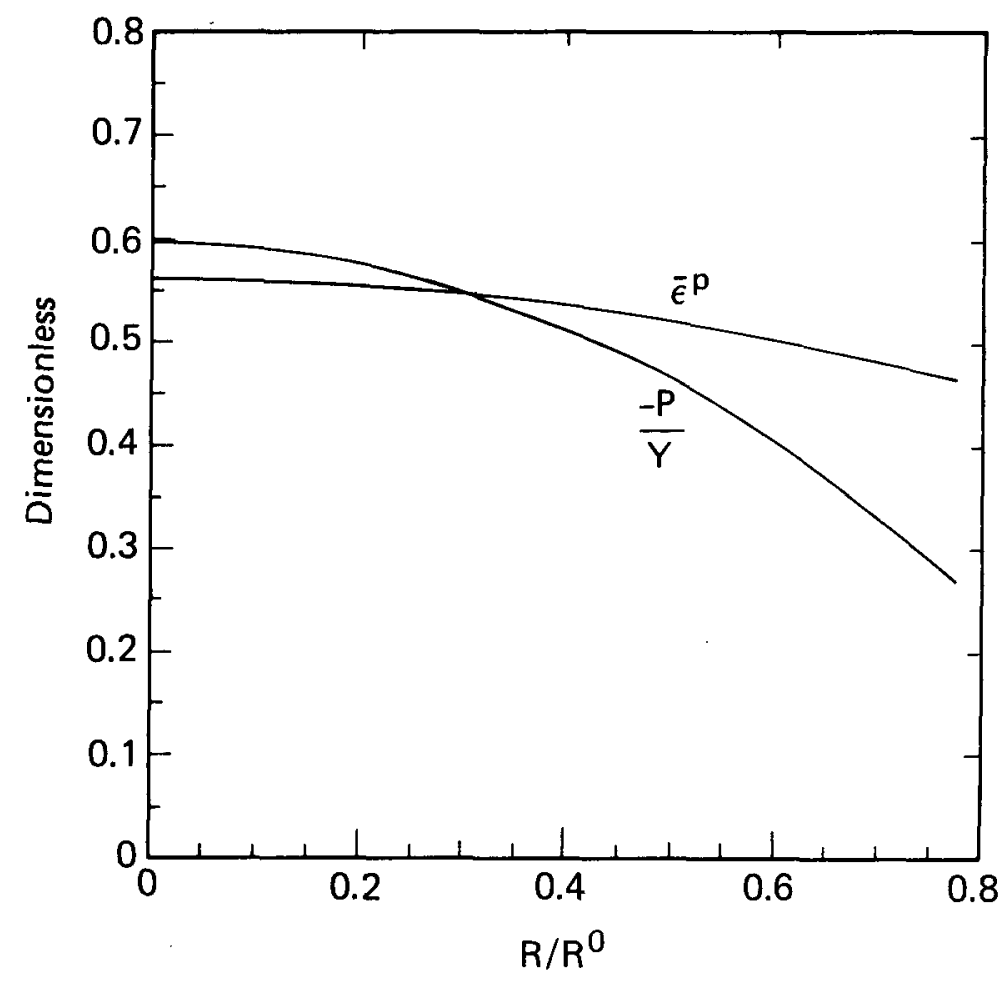

Fig. A.3. Calculated profiles at the cylinder mid-section at time of fracture, $R / R^{0}=0.772$. $\bar{\varepsilon} \mathrm{P}$ is the equivalent plastic strain; $-\mathrm{P} / \mathrm{Y}$ is the ratio of the hydrostatic stress to the flow stress. Both variables are dimensionless.

profiles are not constant. However, Fig. A.3 shows that the equivalent plastic strain profile is fairly flat and that the strain calculated from the external radius, $-2 \ln \left(R / R^{0}\right)=-2 \ln (-0.772)=0.52$, is a good measure of the average value of $\bar{\varepsilon}$ ?

Figure A.4 shows that the axial stress; $\bar{\sigma}_{z z}$; which carries the load, is quite different from the equivalent stress o shown as $\mathrm{Y}$ in Fig. A.4. The true stress from the simulation program, $\bar{\sigma}_{z z}$; was calculated by suming zone by zone the product of the zone stress and zone area in the mid-plane and dividing by the mid-plane area. The calculated value of the true stress corresponding to Fig. A.4 is $\bar{\sigma}_{z z}=66.7 \mathrm{ksi}(460 \mathrm{MPa})$; this is not too different from a mean value of the equivalent stress $\sigma$ eq, taken as $62.4 \mathrm{ksi}(430 \mathrm{MPa})$, where $\sigma_{\mathrm{eq}}=\mathrm{Y}$. Thus the external measurements on a tension test can give stress/strain information suitable for establishing a first guess for a constitutive relation for the plastic work-hardening function. 


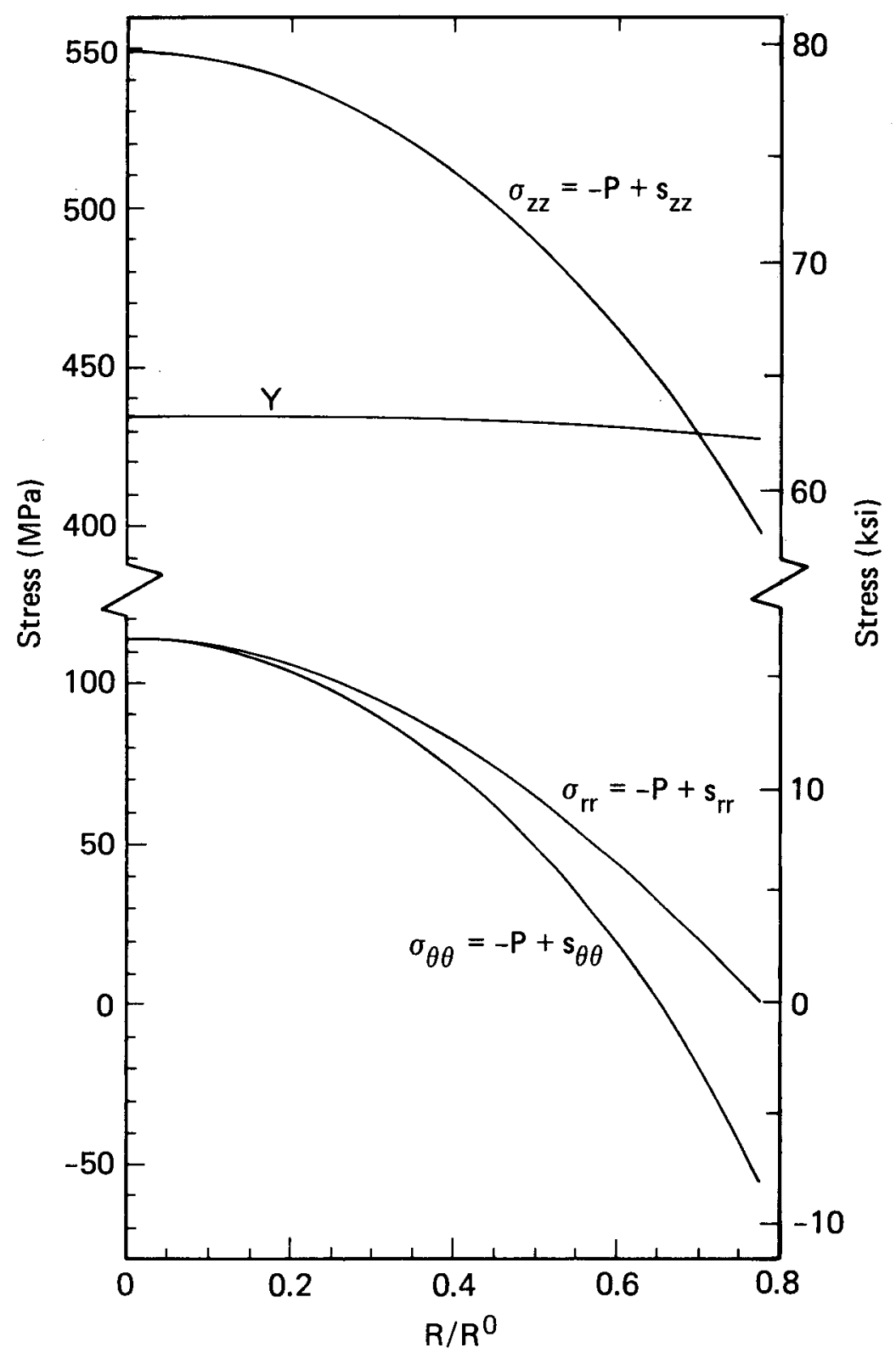

Fig. A.4. Continued from Fig. A.3, $\sigma_{z z}$ is the axial stress, $\sigma_{r r}$ the radial stress, and $\sigma_{\theta \theta}$ the hoop stress. The flow stress $\mathrm{Y}$ is also the equivalent stress $\sigma_{e q}$, since the material is at the elastic limit.

Figure A.5 shows that the hydrostatic stress $-\mathrm{P}$ is responsible for the nonuniform axial stress $\sigma_{z z}$ in Fig. A.4.

For aluminum, the power-law form describes the observed behavior very wel1, and the constants were determined on the first try. For a metal with a more complex work hardening behavior, several iterations of the computer 


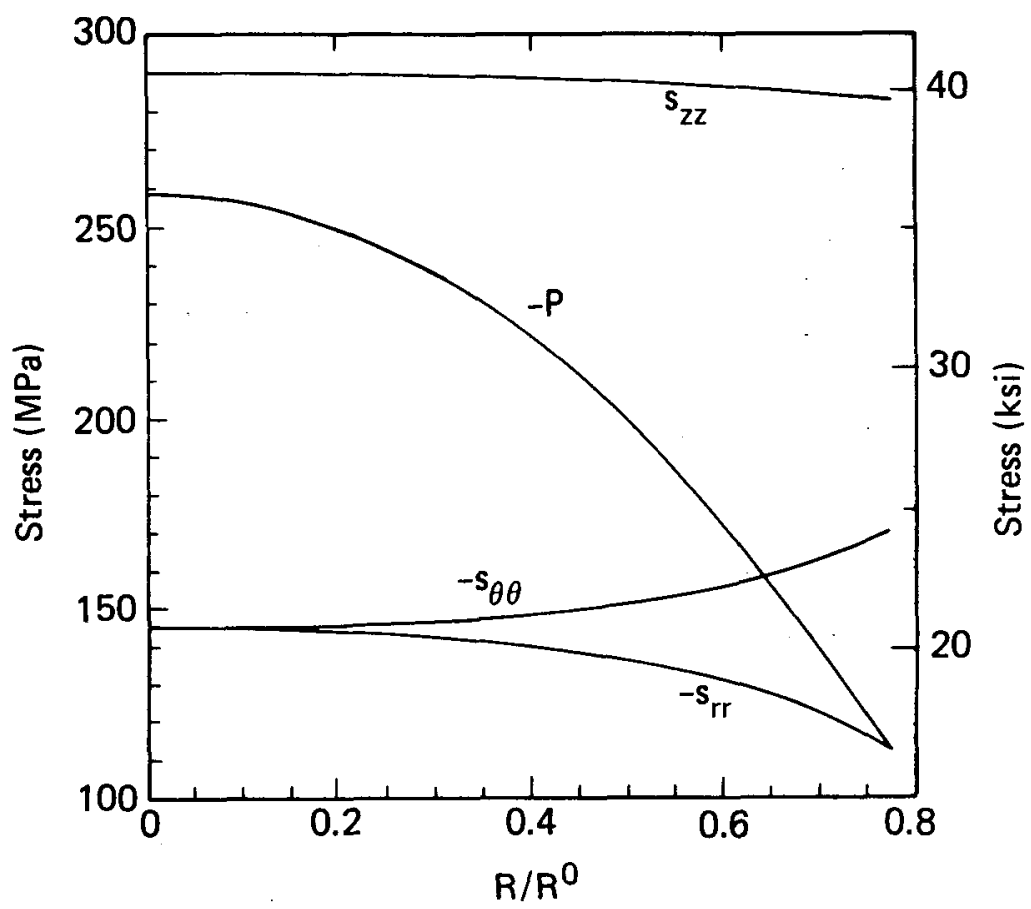

Fig. A.5. Continued from Fig. A.3. $s_{z z}, s_{r r}$ and $s_{\theta \theta}$ are stress deviators; $-P$ is the hydrostatic stress.

simulation program would probably be necessary to develop a satisfactory form to describe the flow stress.

Figures A.6 and A.7 show contours of the axial stress $\sigma_{\mathrm{zZ}}$ and the hydrostatic pressure $P$ at the same radial strain as in Figs. A.3, A.4, and A.5. Figure A.7 shows that the pressure is compressive in a region one to two radii away from the center. This is caused by the free surface boundary conditions on the exterior of the cylinder. The interior stress in the direction normal to the cylinder free surface must be zeru at the free surface. To maintain this stress-free condition, there is motion normal to the free surface as the cylinder is elongated. The net effect is that material moves away from the center region; this is similar to an extrusion process by squeezing.

\section{Impact Experiments}

The impact of a cylinder against a rigid boundary provides a simple geometry in which to examine the behavior of the constitutive model for a different stress/strain history. Figure A.8 shows results of calculations and experiments for the impact of 6061-T6 aluminum cylinders against a rigid 


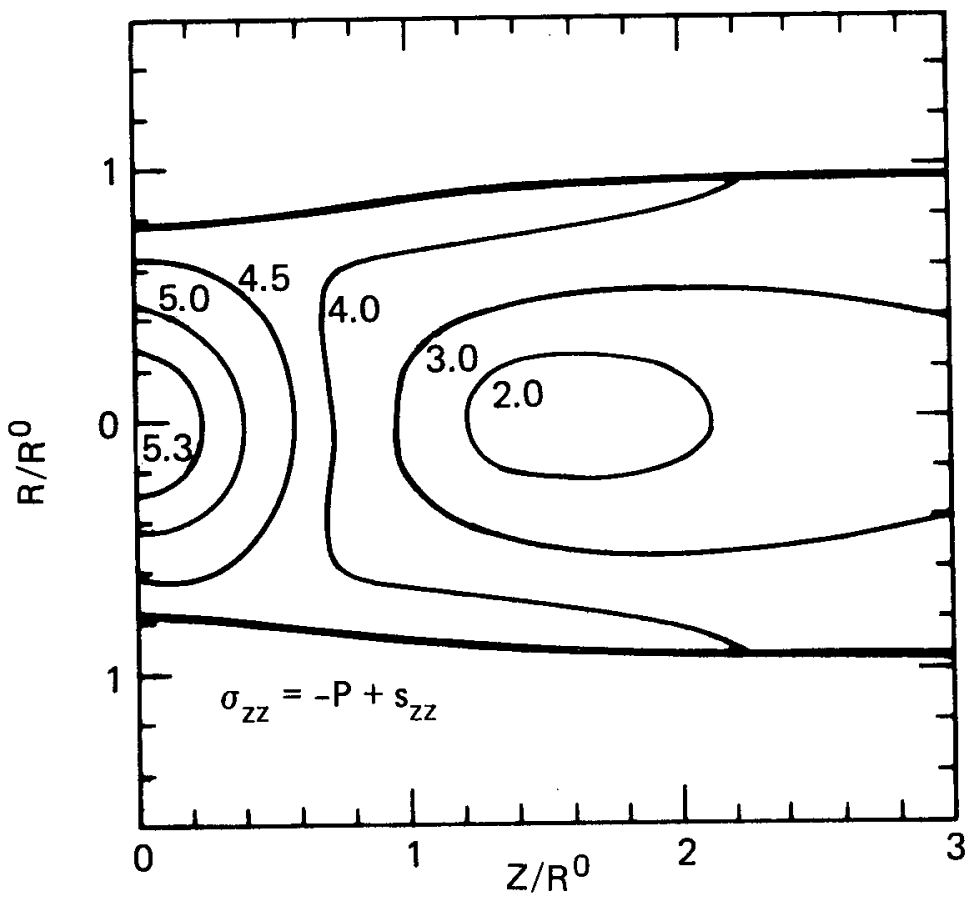

Fig. A.6. Calculated contours of axial stress $\sigma_{z z}$ at time of fracture.

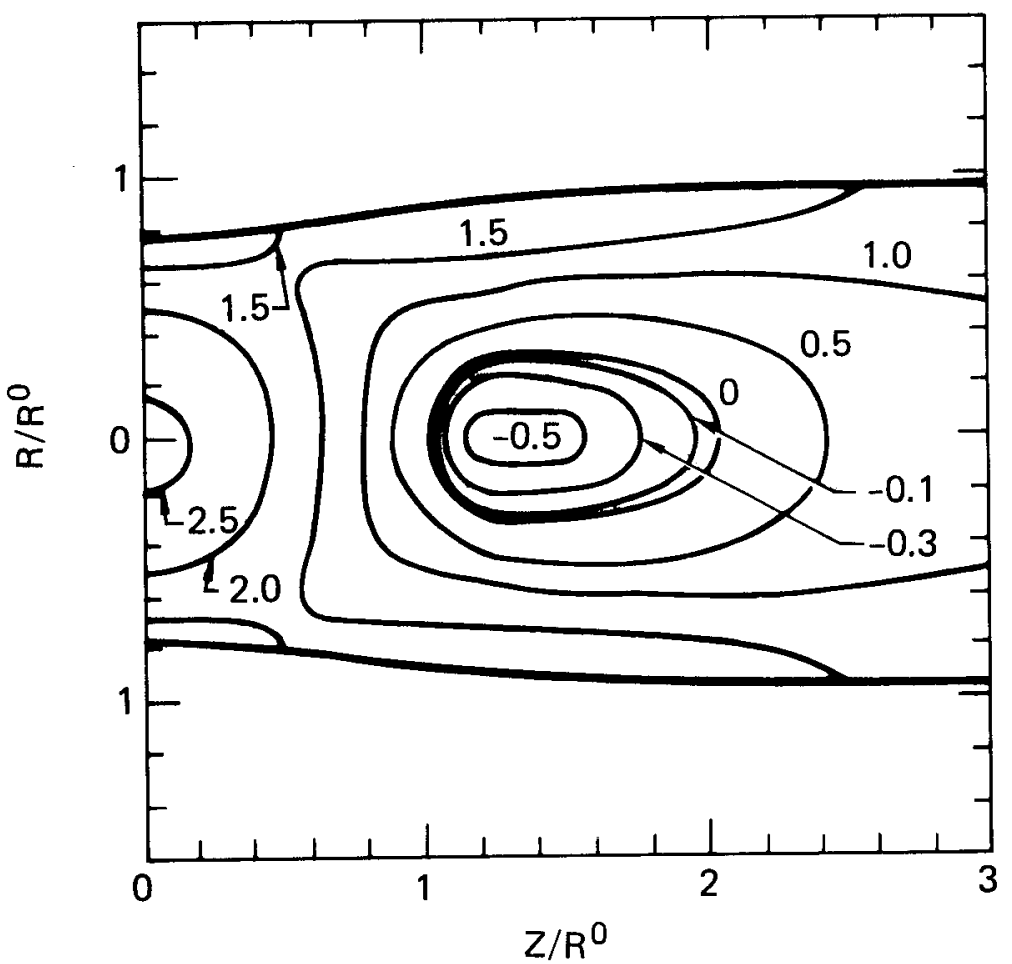

Fig. A.7. Calculated contour of the hydrostatic stress $-P$ at time of fracture. 

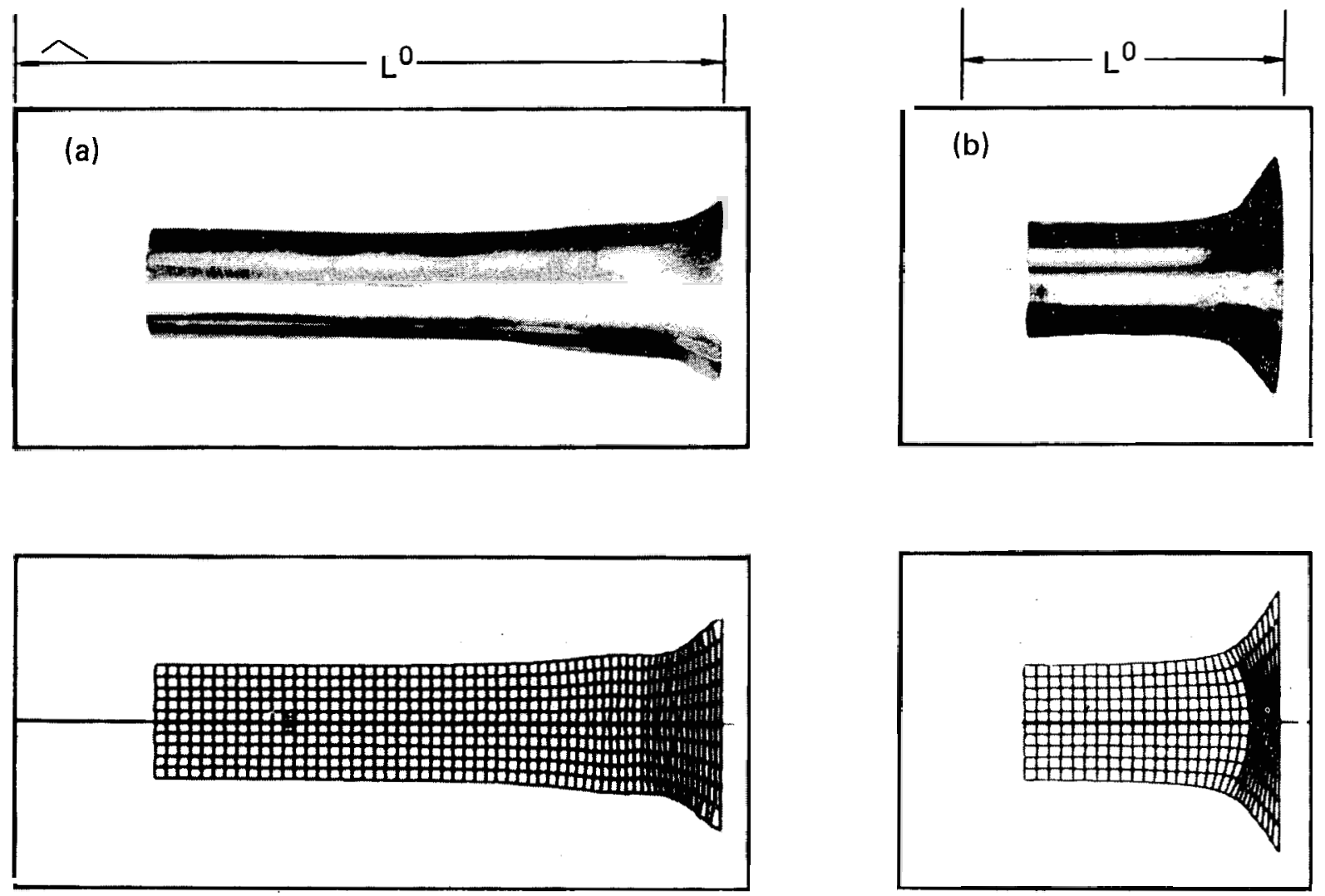

Fig. A.8. Comparison of experiment (top) and calculation (bottom) of right-circular aluminum cylinders after striking a rigid boundary. (a) Original length $\mathrm{L}^{0}=1.85 \mathrm{in}$. $(4.69 \mathrm{~cm})$; final length $\mathrm{L}^{\mathrm{f}}=1.52 \mathrm{in} .(3.86 \mathrm{~cm})$; impact velocity $U=0.0108$ in. $/ \mathrm{s}(0.0275 \mathrm{~cm} / \mathrm{s})$. (b) $\mathrm{L}^{0}=0.925$ in. $(2.35 \mathrm{~cm}) ; \mathrm{L} f=0.650 \mathrm{in} .(1.65 \mathrm{~cm}) ; \mathrm{U}=0.0147 \mathrm{in.} / \mathrm{s}(0.0373 \mathrm{~cm} / \mathrm{s})$.

boundary. The experimental rigid boundary is obtained by backing a 1-in.$(2.54-\mathrm{cm})$-thick alumina tile with a section of hard steel of the same thickness. The final lengths of the cylinders are very sensitive to the flow stress. All the details of the experimental results were closely reproduced by the calculations using the constitutive model described.

The effect of temperature will become important at higher impact velocities. Additional experiments would be required to map out the regions of thermal softening. When the melting temperature is reached the flow stress $Y$ must be set to zero.

\section{Torsion Tests}

The stress-loading conditions in the simple tension test are seldom the same as those under which materials fracture in an engineering structure. In 
terms of the principal stress deviators $s_{1}, s_{2}$, and $s_{3}$, the loading of a simple tension test is $s_{2}=s_{3}=-s_{1} / 2$, where $s_{1}$ is in the direction of the tension load. We use the usual definition $s_{1} \geq s_{2} \geq s_{3}$, with the stress deviators positive in tension. Analysis of tests used to evaluate fracture resistance, such as the compact-tension, Charpy, and notched-cylinder tests, shows that the stress is very close to pure shear where maximum strain occurs. For pure shear, the stress loading is $s_{3}=-s_{1}$, with $s_{2}=0$. It is not possible to make an accurate direct measurement of the strain in the foregoing fracture-toughness geometries, so the plasticity function cannot be checked. However, the torsion test provides a relatively simple geometry where $s_{1}=-s_{3}$ and $s_{2}=0$.

The torsion specimens used in this investigation are cyindrical, with a reduced central section that confines the area of plastic deformation. The central region has a diameter of $0.75 \mathrm{in} .(19 \mathrm{~mm})$; the diameter increases to $2.0 \mathrm{in.}(51 \mathrm{~mm})$ in the bulk section. There is a 3 -in. radius of curvature between minimum and maximum section. The torque loading was applied using a constant rate of overall twist (i.e., stroke control) while the axial load was maintained at zero (1.e., load control). Data were recorded in three independent ways: (1) as a plot of torque vs angle of twist (e.g.: Fig. A.9); (2) as a sequence of still photographs taken during the test with an emphasis on determining the strain immediately before fracture; and ( 3 ) as a video recording of the entire test. Vertical lines were lightly scribed into the specimen surface to facilitate photographic measurement of the local shear twist angle. The test methods were correlated in time, so that cross-referencing the data provided verification of the test methods. Failure corresponded to simultaneous loss of torque-carrying capacity and cracking on the surface of the specimen. The fracture surface was very flat in every case, indicating a pure shear across the failed section (tensile load successfully maintained at zero).

The results at failure were very consistent from test to test with respect to both the maximum torque (Fig. A.9) and the localized shear twist angle (Fig. A.10). Further, microstructural examination (i.e., examination of polished sections) of the grain structure just below the failed surface showed grain shear strain approximately equal to that observed on the surface. The shear strain was also measured at various depths using the microstructural approach. A plot of shear angle at failure vs radius (Fig. A.11) shows that 


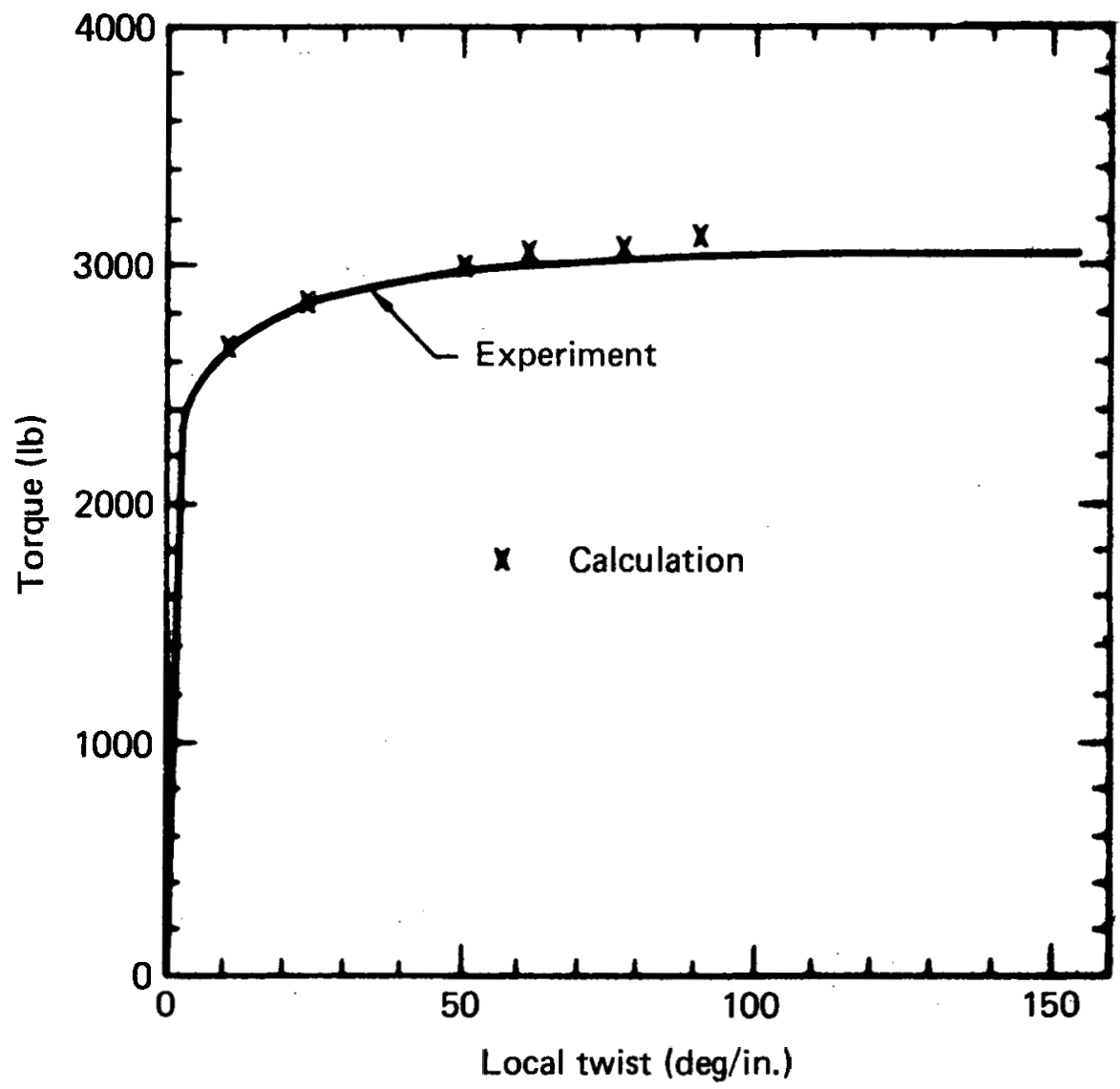

Fig. A.9. Torque and angle of twist for a solid 6061-T651 aluminum cylinder.

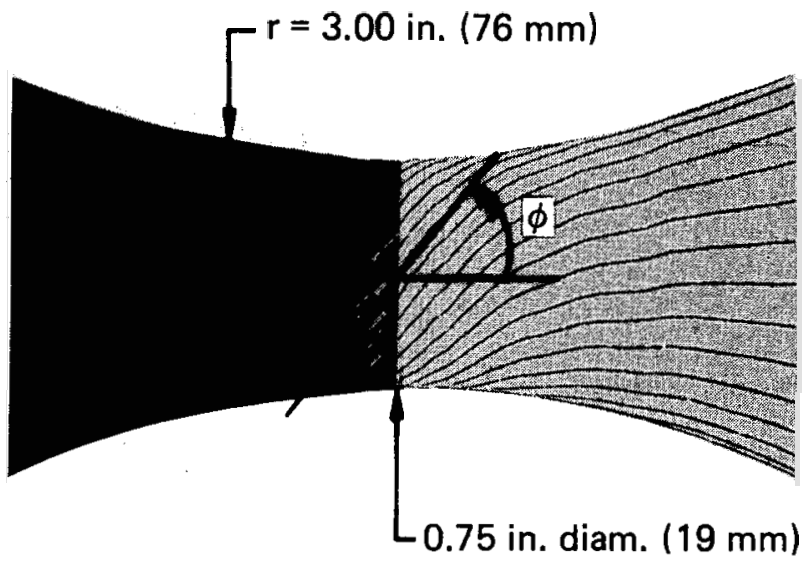

Fig. A.10. Torsion test of a solid 6061-T651 aluminum cylinder. On the left is the experiment, with scribe lines that were originally parallel to the cylinder axis. On the right is the calculation, with lines formed from the Lagrangian coordinates. 


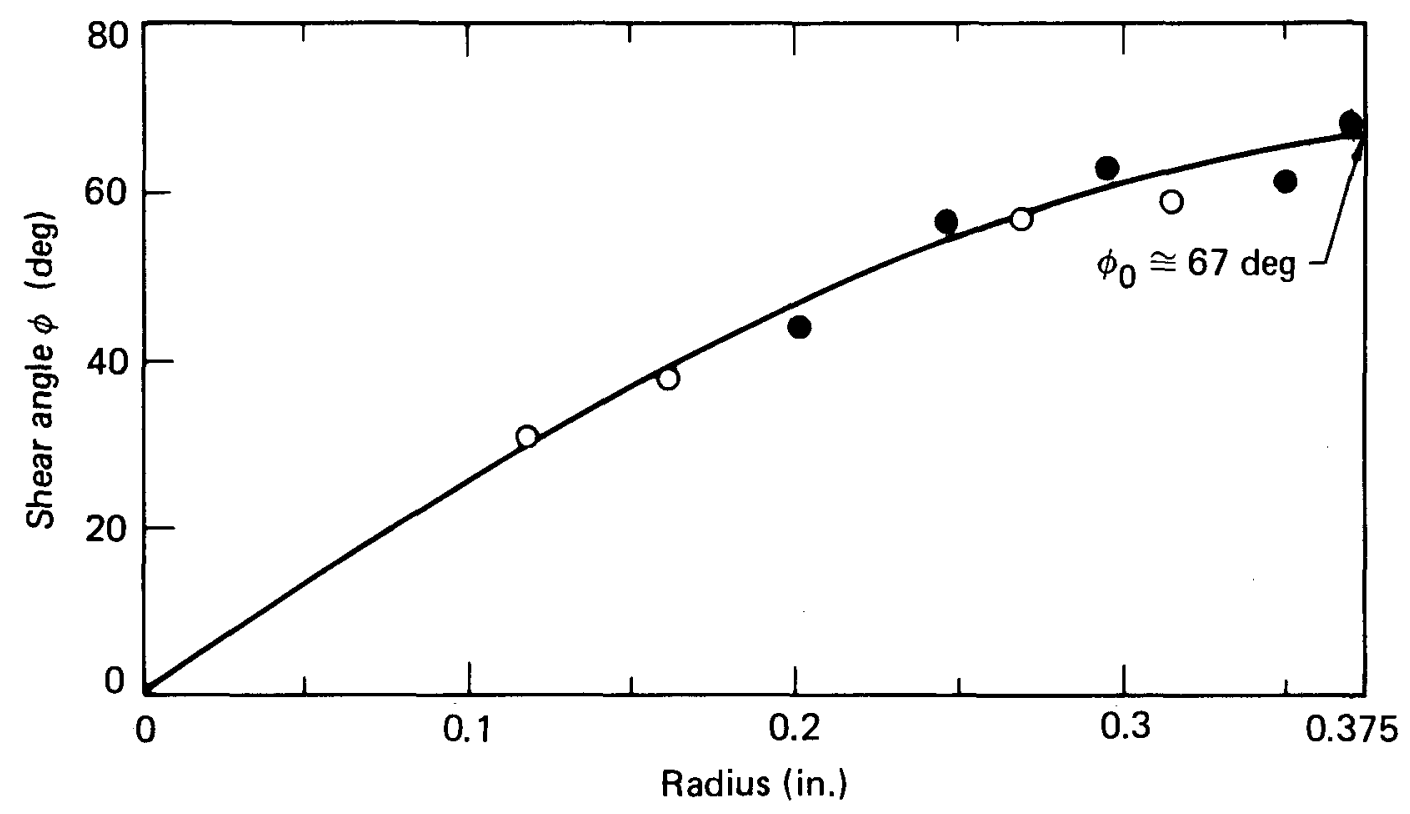

Fig. A.11. Variation of shear angle, measured from the angle of grain rotation, as a function of radius in 6061-T651 aluminum torsion test. A nonlinear strain distribution is observed, as predicted by the computer analysis. The angle $\phi_{0}$ is the shear angle measured on the surface.

the strain distribution is nonlinear. This nonlinear behavior was predicted in the computer simulation.

As in the tensile-specimen study, the flow stress as a function of equivalent plastic strain can be determined from the experimental results of Fig. A.9. Since a power-law plasticity function is in widespread use, we apply this form to express the experimental results:

$$
Y=a\left(1+b \bar{\varepsilon}^{p}\right) c ;
$$

here $Y=$ flow stress, $\bar{\varepsilon}^{P}=$ equivalent plastic strain, and $a, b$, and $c$ are material constants. With the constants for the plasticity function estimated from experiment, the torsion test can be simulated on the HEMP finite-difference simulation program.

Figure A.10 compares calculation and experiment just before ductile fracture of the solid cylinder. Figure A.12 shows experimental values of stress and strain compared to the plasticity function given above with $a=35.4 \mathrm{ksi}(244 \mathrm{MPa}), \mathrm{b}=10^{3}$, and $\mathrm{c}=0.051$. The corresponding parameters derived from simple tension tests are $a=38.4 \mathrm{ksi}(265 \mathrm{MPa})$, 


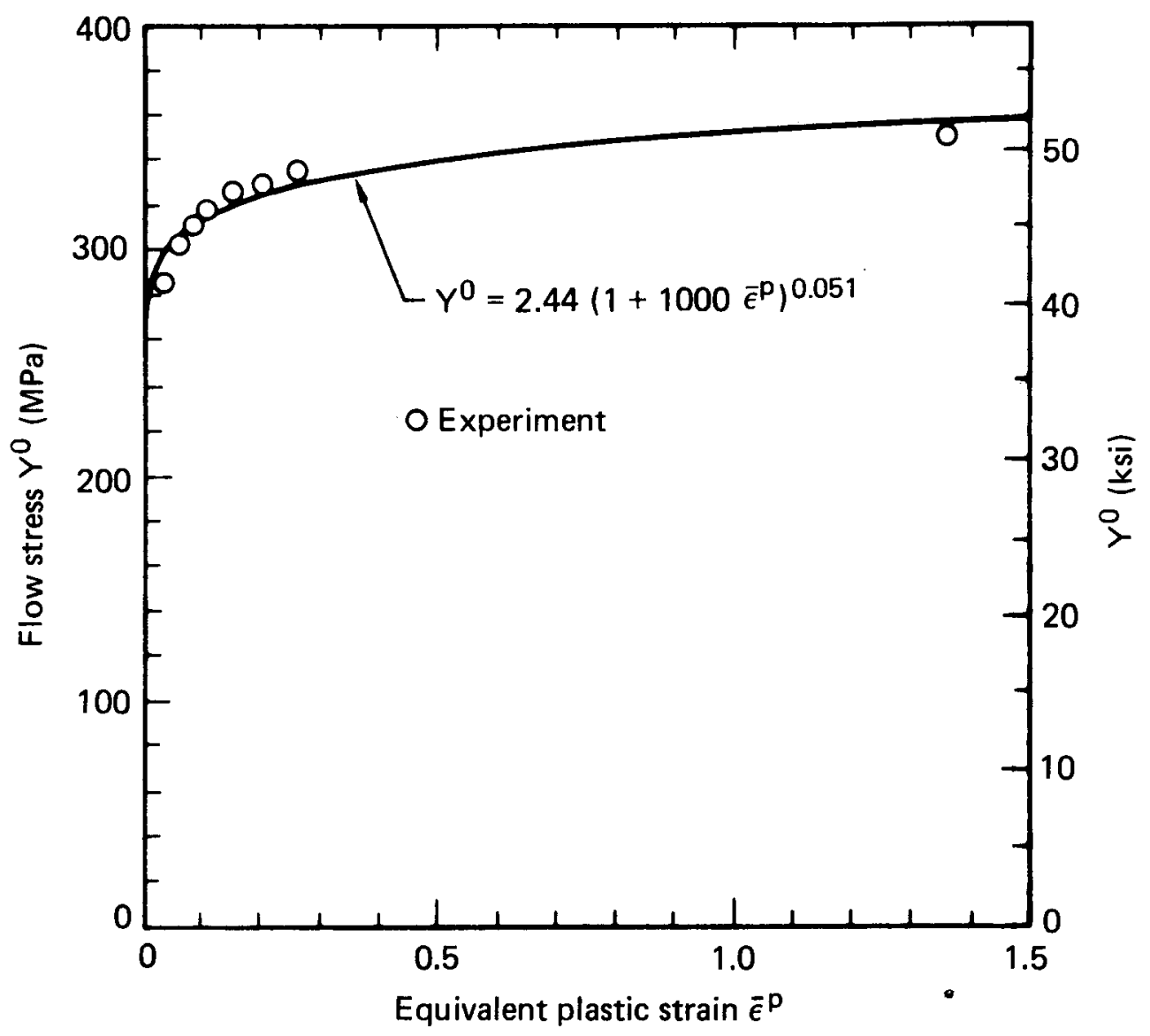

Fig. A.12. Flow stress obtained from solid-cylinder torsion test of 6061-T651 aluminum.

$b=125$ and $c=0.1$. Thus, the elastic limit for the torsion test occurs at $35.4 \mathrm{ksi}$ (244 MPa) and for the simple tension test at $38.4 \mathrm{ksi}(265 \mathrm{MPa})$. A more significant difference is the work-hardening exponent, equal to 0.051 for the torsion test and 0.1 for the simple tension test.

Although the specimen-to-specimen variation of torque and shear twist angle was very smal1, the torque predicted using a von Mises yield surface was about $15 \%$ higher than the experimental values. In addition, the localization of twist is much less pronounced in the computer simulation than in the actual specimens. While such discrepancies are of concern, the fact that the von Mises yield condition does not necessarily represent material behavior under large strains has been previously reported. ${ }^{24}$ The torsion simulation was rerun, however, using a simple approximation to the Tresca yield surface. The difference between the von Mises and Tresca criteria in pure shear reduces the 
simulated torque by about $15 \%$ (i.e., by $2 / 3^{l / 2}$ ). Furthermore, the twist localization experienced in the test specimens can be better reproduced using the Tresca simulation, although there was still some discrepancy in this respect.

A second area of concern is adequate simulation of shear deformation involving large rotation, as in the torsion test. Hayhurst and Storakers 25 discuss the use of constitutive and damage relationships in situations in which rotations of material elements occur within a homogeneous stress field. They observe an apparent strengthening in copper samples; they ascribe this to the rotation of the surface elements of the material, which have undergone material deterioration due to the maximum principal stress, to new orientations in which they are subjected to lower values of direct tensile stress. A comparison of the computer-generated failure geometries with the actual specimens indicates that the simulation correctly describes rotation. An axially loaded flat-plate geometry (one that also results in shear failure) was also tested to investigate shear damage with limited rotation. These results are presented in subsequent sections.

A characteristic of the solid torsional specimen is the nonuniform shear through the thickness. This tends to mask the true shear stress-strain behavior by shifting the relative shear-load capacity from the outer fibers to the inside during plastic deformation.

Thus, while the solid-cylinder torsion test gave accurate values of flow stress and plastic strain at failure, this test is not sensitive enough to determine either the elastic limit or the work hardening at plastic strains of less than $30 \%$. Thin-walled hollow cylindrical specimens with nearly uniform shear in the wall were used to evaluate the shear stress-strain relation, as shown in Fig. A.13.

These tests confirmed the Tresca yield model for pure shear. However, because of geometrical constraints imposed by the test apparatus, a final failure strain could not be evaluated from the hollow specimens. The maximum plastic strain that was still uniform around the circumference was 20 to $30 \%$. Up to this 1 imit, the test is sensitive enough to determine the work hardening.

The best $\mathrm{fit}$ to the function used earlier was obtained with a $=35.4 \mathrm{ksi}$ $(224 \mathrm{MPa}), \mathrm{b}=1000, \mathrm{c}=0.073$. Figure A.14 shows the work-hardening function and data points reduced from the experimental torque-twist records and 


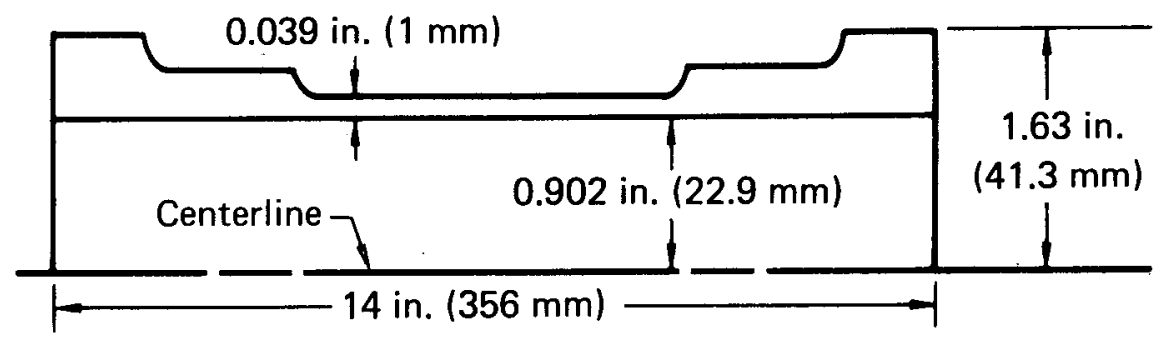

Fig. A.13. Dimensions of the hollow cylinder for the torsion test.

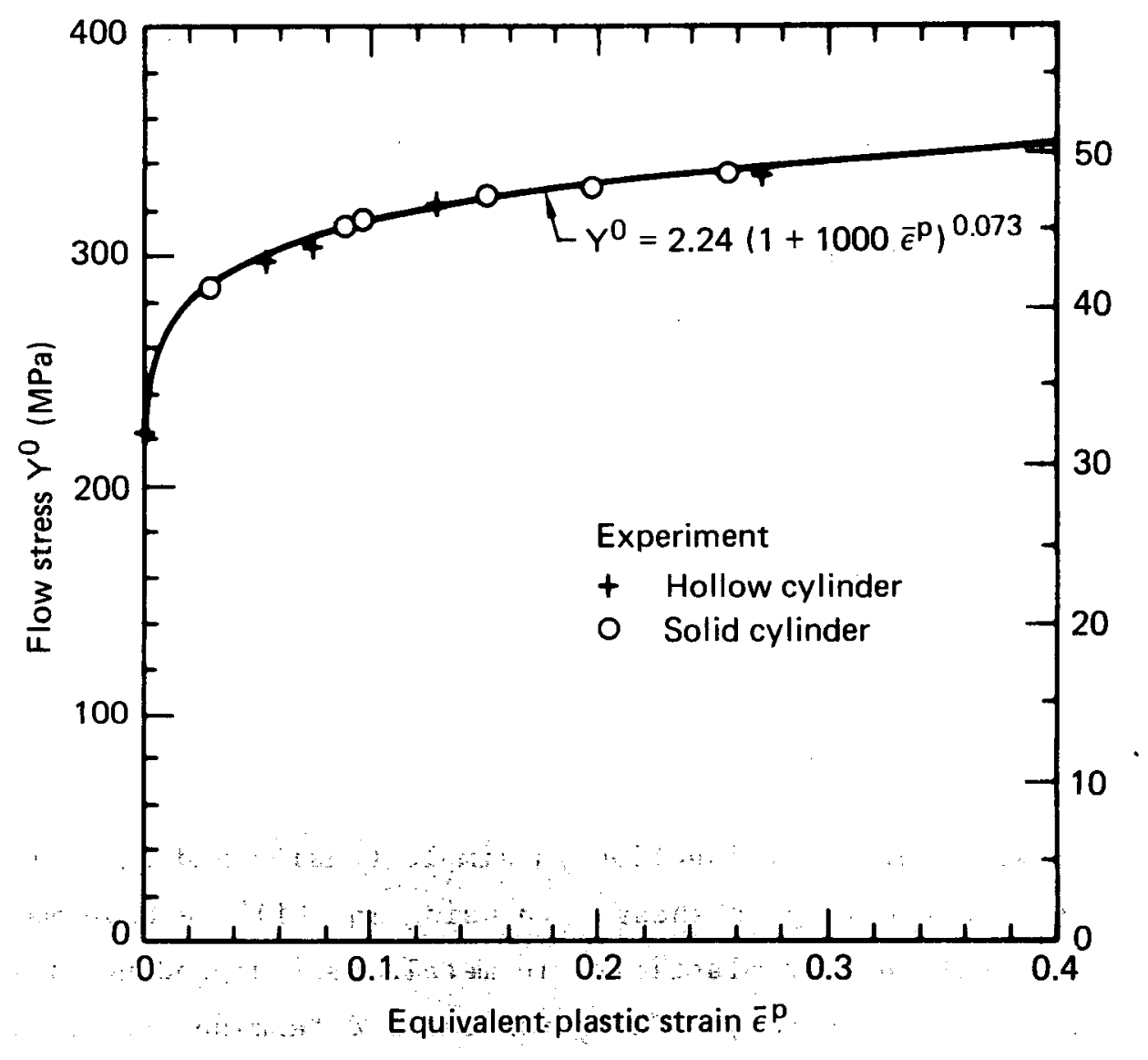

Fig. A.14. Flow stress derived from solid and hollow cylinders of 6061-T651 aluminum. 
photographs of the scribe lines taken during the test. The figure shows that the work hardening of this aluminum in torsion is not well represented by a simple power law over the entire range of plastic strain. Indeed, at large plastic strains the work-hardening exponent is less than about 0.03 .

\section{Plasticity Function for Combined Stress Loading}

The stress state at fracture in fracture-resistance test specimens is neither pure tension nor pure shear. To simulate fracture tests thus requires a work-hardening function that depends on the state of stress. We first introduce a parameter $A$ that characterizes the stress field. In the simple tension test, $s_{2}=s_{3}$; in the torsion test, $s_{2}=0$. We define $A=$ $s_{2} / s_{3}$, so that $A$ ranges from 1 to 0 as the stress changes from simple tension, which we term symmetric loading, to pure shear, which we term asymmetric loading. A general definition of the parameter $A$, which measures the degree of asymmetry of the loading, including both tension and compression, is

$$
A=\max \left(\frac{s_{2}}{s_{3}}, \frac{s_{2}}{s_{1}}\right)
$$

A work-hardening function that is convenient for numerical calculations and that uses our work-hardening data from the tension and torsion tests directly is

$$
\mathrm{Y}=\dot{\mathrm{Y}}_{\mathrm{T}}\left(\bar{\varepsilon}^{\mathrm{P}}\right) \mathrm{A}^{\lambda}+\mathrm{Y}_{\mathrm{S}}\left(\bar{\varepsilon}^{\mathrm{P}}\right)\left(1-\mathrm{A}^{\lambda}\right)
$$

Here $Y_{T}$ is the work-hardening function in simple tension and $\mathrm{Y}_{\mathrm{S}}$ is the work-hardening function in pure shear. In using Eq. (1), we have made the conventional assumption that plasticity in metals is independent of the mean stress; we also assume isotropy. The parameter $\lambda$ determines the shape of the yield surface. With $\wedge=0$, we recover the von Mises surface. If $\mathrm{Y}_{\mathrm{S}}=3^{1 / 2} \mathrm{Y}_{\mathrm{T}} / 2$ and $\mathrm{A}=1.593$ we obtain an excellent approximation to the Tresca surface. We show the intersection of these two surfaces with the plane of constant mean stress, $s_{1}+s_{2}+s_{3}=0$, in Fig. A.15.

We use the Levy-Mises flow rule, so that the principal plastic strain rates are proportional to the principal stresses. This is especially 


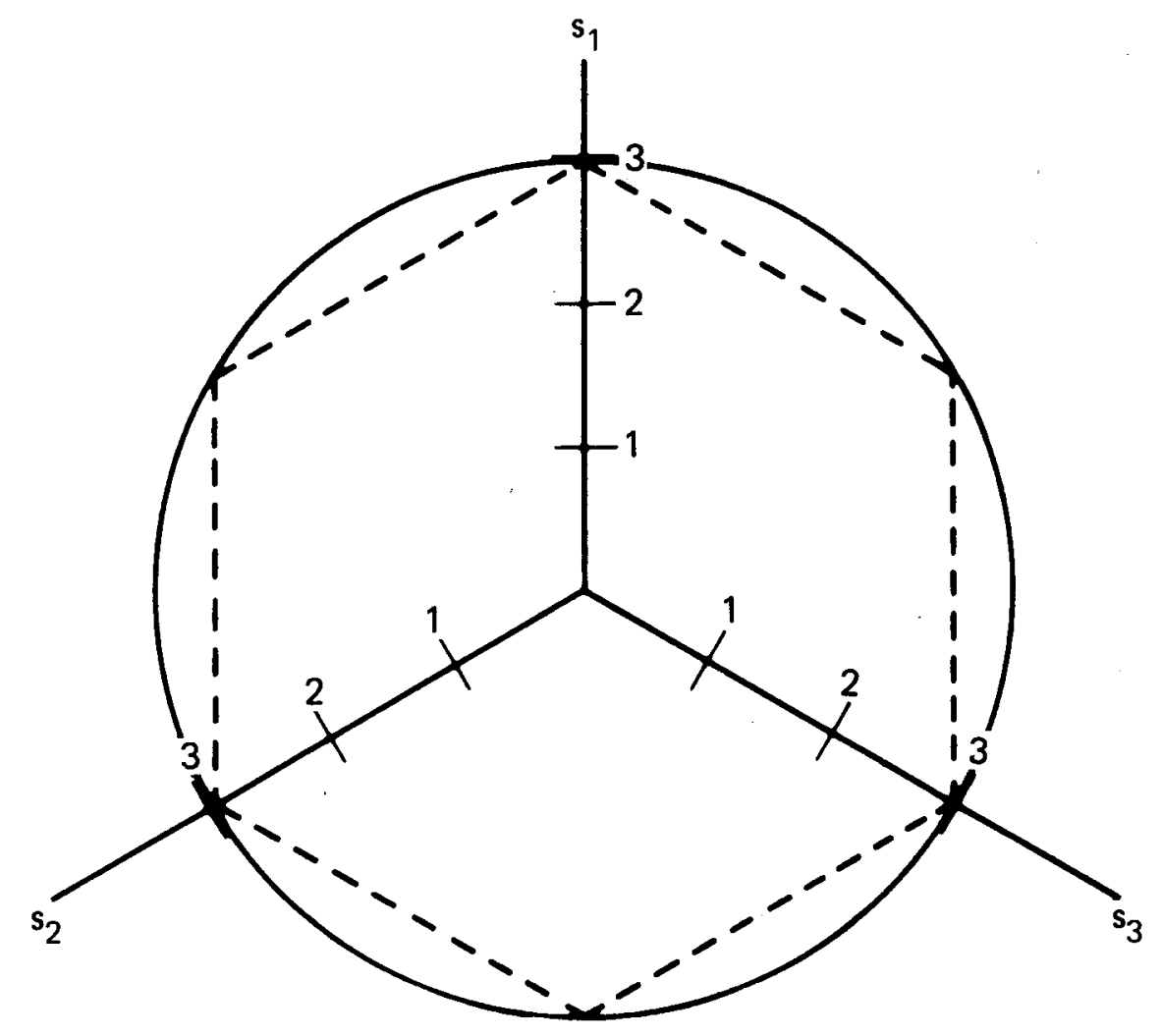

Fig. A.15. Intersection of yield surface with plane $s_{1}+s_{2}+s_{3}=0$. Solid line: $\lambda=0 ; Y_{T}$ $=\mathrm{Y}_{\mathrm{S}}=43.5 \mathrm{ksi}(300 \mathrm{MPa})$. Dotted 1 ine: $\lambda=1.593 ; \mathrm{Y}_{\mathrm{T}}=43.5 \mathrm{ksi}(300 \mathrm{MPa}), \mathrm{Y}_{\mathrm{S}} 37.7$ ksi $(260 \mathrm{MPa})$.

convenient in our numerical solutions, and it satisfies existence and uniqueness requirements, just as the associated flow rule does (see, for example, Ref. 22). In addition, yield surfaces with corners have the advantage that there is no ambiguity in the direction cosines of the principal plastic strain rates. For arbitrary $Y_{T}, Y_{S}$ and $\lambda$, the surface we define is not necessarily dissipative. We note that the requirement of convexity of the yield surface is a sufficient, but not a necessary, condition for satisfying Drucker's postulate (that yielding be locally dissipative) when work hardening is present. ${ }^{23}$ Thus, although the yield surfaces we will present are not convex, we verified that they were dissipative in the cases tested. 
It is natural to look for a test in which plasticity occurs under combined tension and shear. As stated earlier, this is the loading of practical interest where the resistance-to-fracture properties of material are assessed. A major difficulty is to find a geometry that permits accurate strain measurements as the load increases. A geometry that exhibits combined stress conditions is the tension test of a flat plate. The strain can be monitored by scribing lines perpendicular to the loading direction, as shown in Fig. A.16. The increase in the separation of adjacent lines, divided by the original separation, gives a measure of the strain for positions along the plate. Once again, we used 6061-T651 aluminum in the investigation.

Figure A.17 shows experimental results just before fracture of a flat plate. The strains, determined as just described, are plotted at the geometric center between adjacent lines. The figure also shows computer simulations of the experiment using the work-hardening function determined from a simple tension test and the work-hardening function that depends on the stress state. For this latter calculation the parameter $\lambda$ (from the previous section) had the value 0.7 . We used the work-hardening developed from the hollow-cylinder test for $Y_{S}$. Figure A.18 shows the computed equivalent plastic strain using the two work-hardening functions. The equivalent plastic strain is very similar to the strain determined by the change in separation of lines on the surface, shown in Fig. A.17. Figure A.19 shows the intersection of this surface with the plane $s_{1}+s_{2}+s_{3}=0$; it also shows the trajectory of the central point of the plate.

The two calculations are compared with the experiment at the strain determined by a $1.00-i n .(25.4-\mathrm{mm})$ clip gauge across the geometric center of the specimen (Fig. A.16). The strain corresponding to Fig. A.17 is $\Delta \mathrm{L} / \mathrm{L}^{0}=0.118$, where $\Delta \mathrm{L}=$ increase in length of $\mathrm{L}^{0}$ and $\mathrm{L}^{0}=$ gauge length $=1.0 \mathrm{in} .(25.4 \mathrm{~mm})$.

The experimental load vs strain as measured by the clip gauge is reproduced by both calculations. Hence using a single strain gauge to correlate this experiment with a calculation would not reveal the error in the plasticity function. 


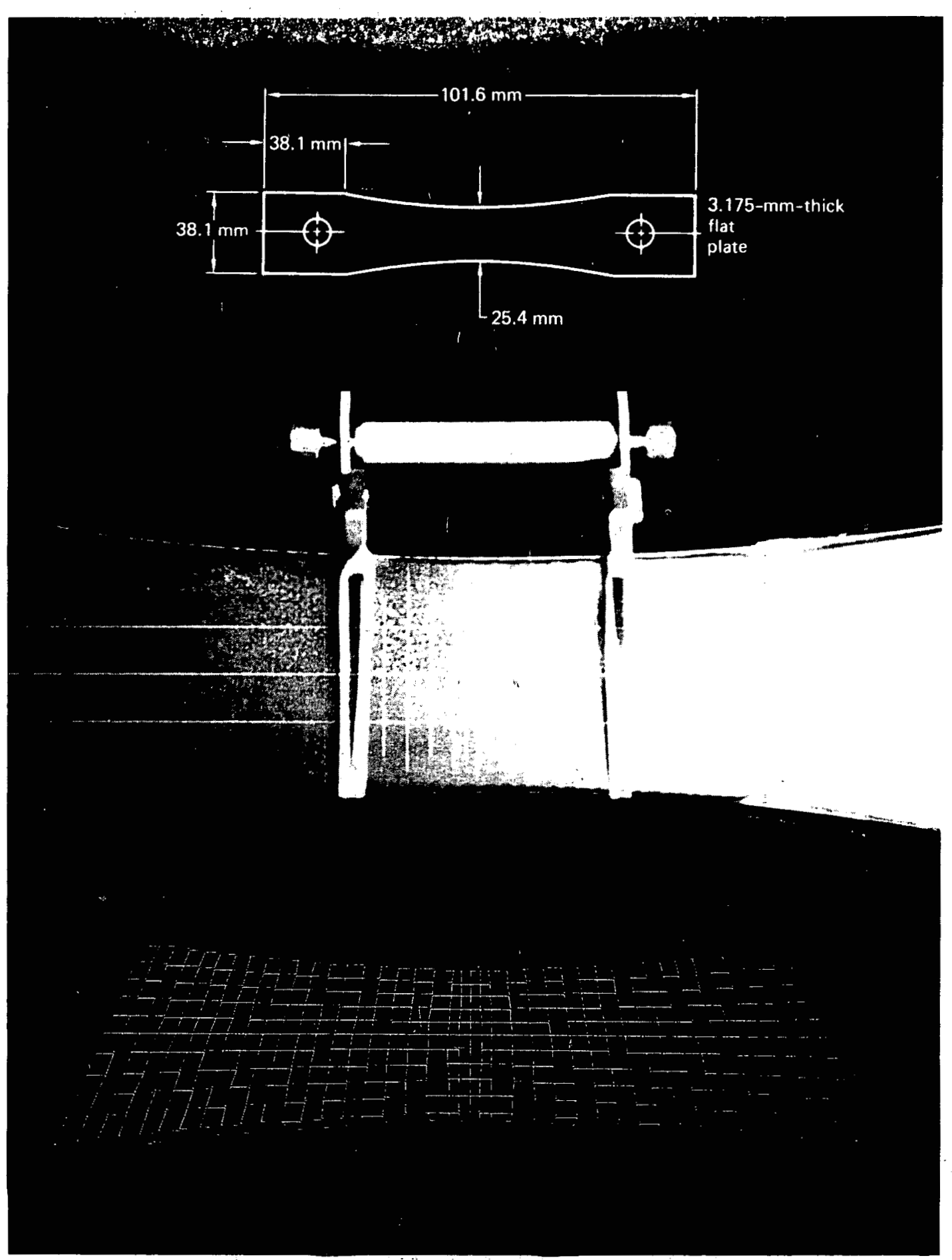

Fig A.16. Tension test of flat plate. At the top is the plate geometry. In the center is a 6061-T651 aluminum plate specimen with scribe lines and 1.00-in. (25.4-mm) clip gauge as tested. At the bottom is the grid used in the computer simulation. 


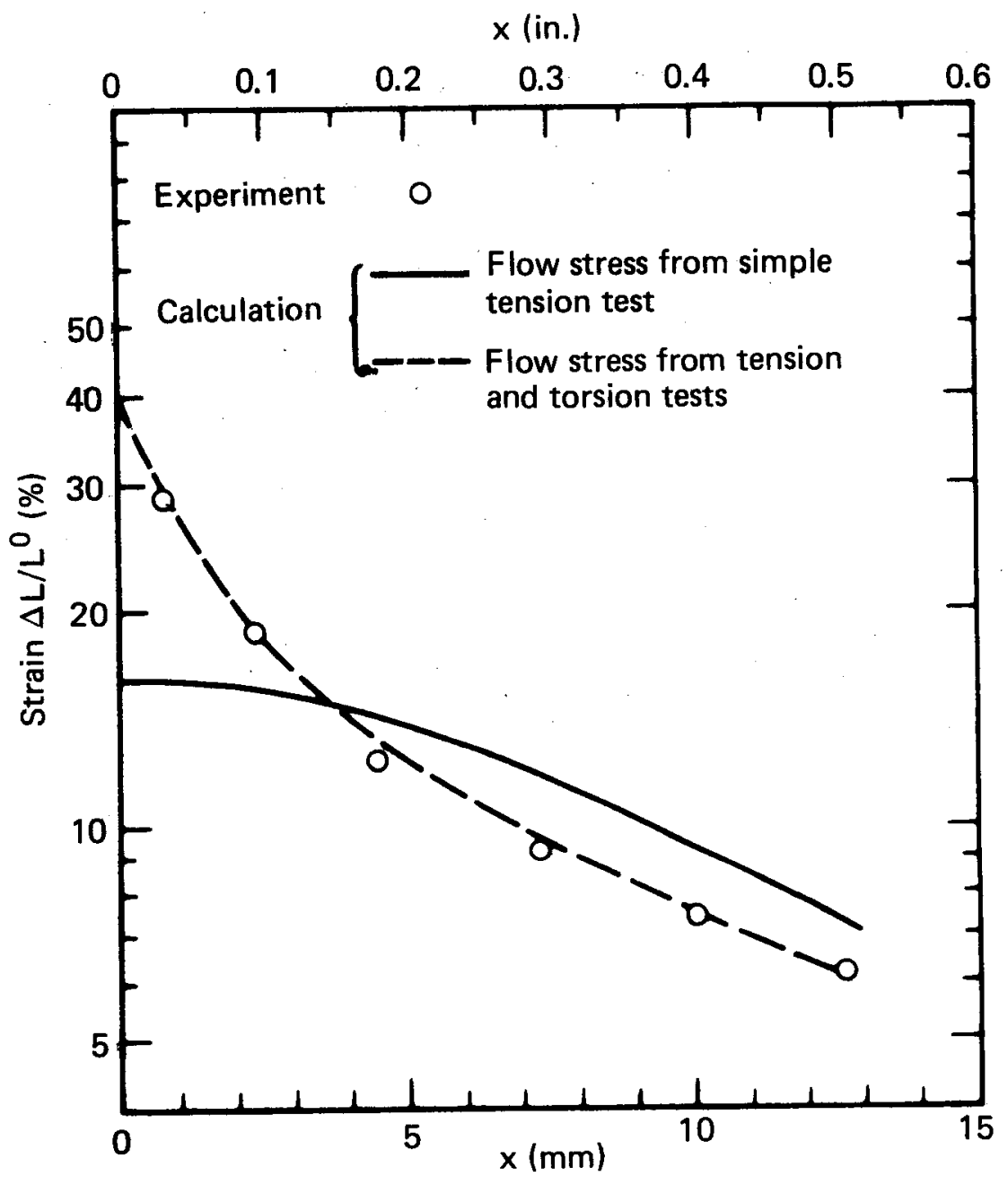

Fig. A.17. Strain along centerline of flat-plate tension test at fracture. $\Delta \mathrm{L}=$ elongation between adjacent scribe lines; $L^{0}=$ original separation of adjacent scribe lines. 


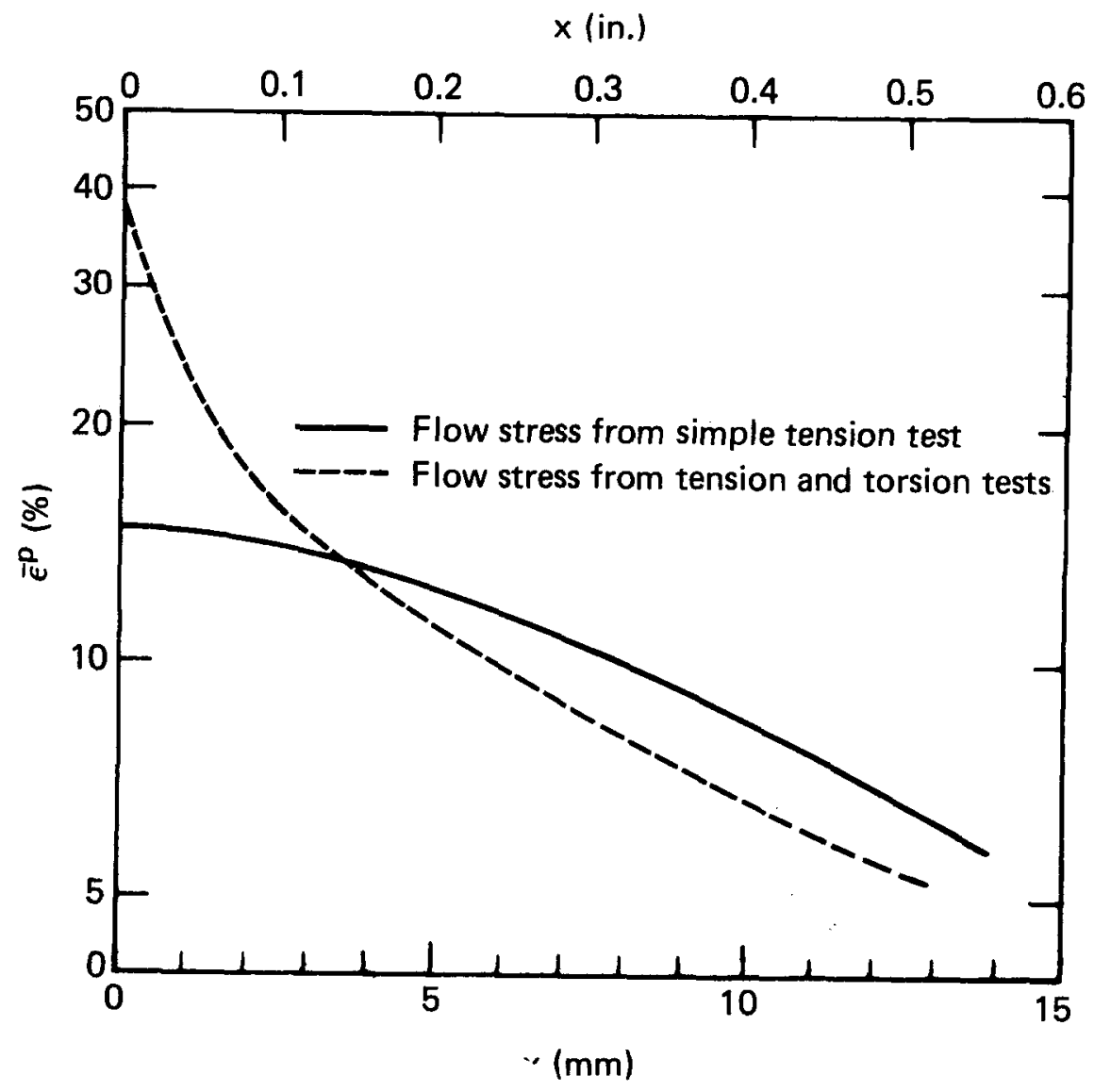

Fig. A.18. Calculated equivalent plastic strain along centerline of flat plate of Fig. A.16. 


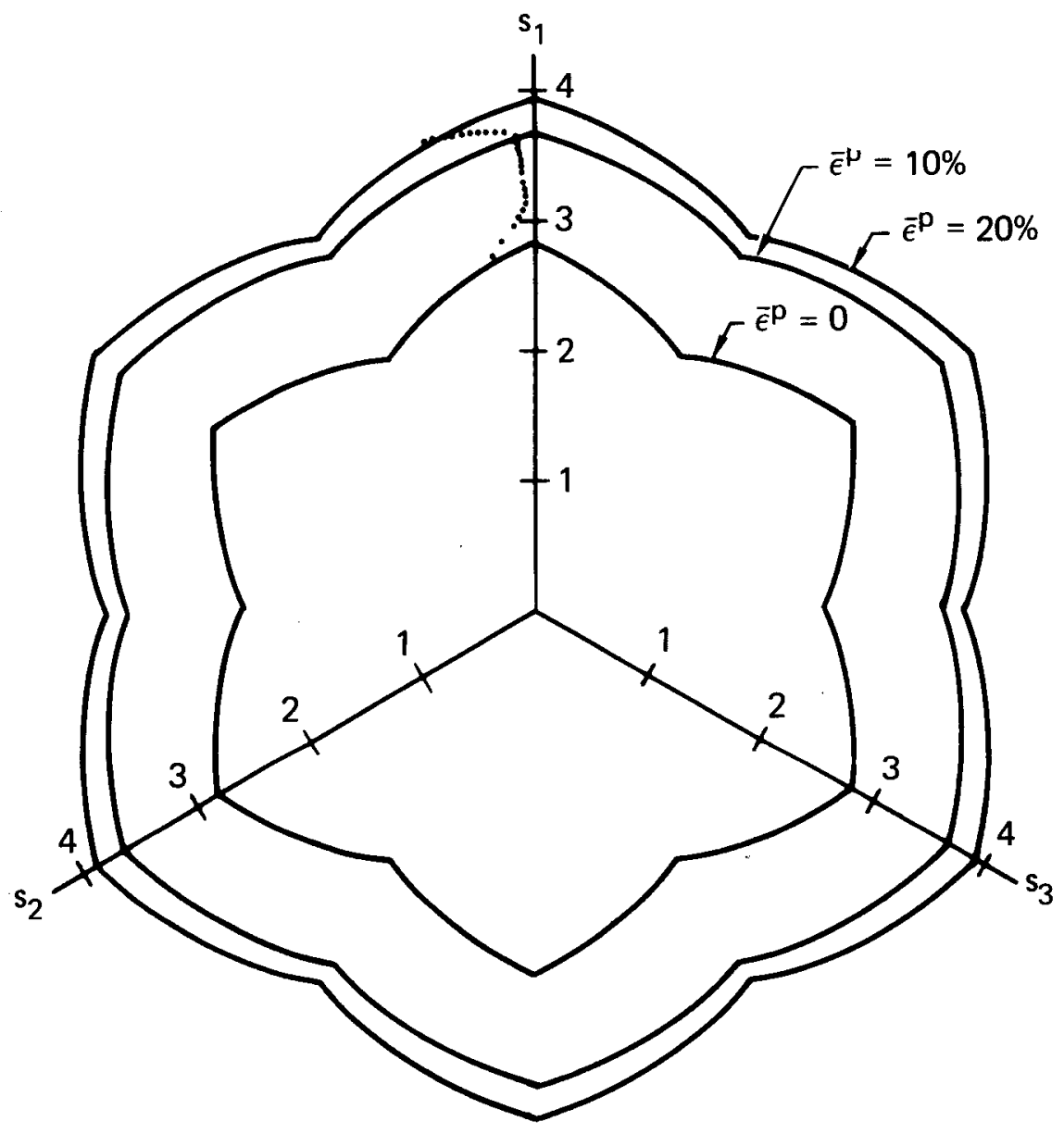

Fig. A.19. Intersection of yield surface with the $\mathrm{p}$ lane $s_{1}+s_{2}+s_{3}=0$ for $\bar{\varepsilon}=0,10$, and $20 \%$. Dotted line shows path of the deviatoric stresses at the geometric center of the thin plate. 
Notched Cylinders

We used the notched, round-bar tension test of 6061-T651 aluminum to assess flow behavior and ductile fracture resistance under conditions of high localized strain (i.e., high strain gradient) and enhanced hydrostatic stress. The next section gives a more complete discussion of results from a series of experiments and computer simulations on geometrically scaled notched-tension specimens. In this section we simply compare the simulation results to the experiment as a verification of the flow model. The strain fields have the form $\bar{\varepsilon}^{\mathrm{P}}=\bar{\varepsilon}_{\mathrm{N}}^{\mathrm{P}} \exp (-\mathrm{ar})$. Here $\bar{\varepsilon}^{\mathrm{P}}$ is the equivalent plastic strain at position $r, \bar{\varepsilon}_{N}^{p}$ is the strain at the notch, $r$ is the distance below the notch, and $a$ is a constant. In the simulations of the notched-cylinder tension tests, we used the work-hardening function from the simple tension test. We have recalculated the tests with the yield surface of Eq. (1) with the same parameters as used for the flat plate. The computer simulation was correlated with the experiment just before fracture by matching the notch profile, as shown in Fig. A.20. The plastic-strain fields for simulations with the flow stress determined from the tension test and the flow stress of Eq. (1) were quite similar, except that the slope of the strain field calculated with Eq. (1) was about $10 \%$ less. Figure A.21 shows results for the notched cylinder with an initial root radius $R=0.157 \mathrm{in} .(4 \mathrm{~mm})$.

\section{Conclusions}

Our experiments show that the work-hardening of 6061-T651 aluminum is different in tension and torsion. By comparing numerical simulations with experiments on flat plates, we find that the work-hardening and stress states are different from either tension or torsion. We have developed a flow-stress model that accounts for these differences, and have used it in a numerical simulation of a notched-round-bar tension experiment. In this geometry, neither the plastic strain nor the equivalent stress are conveniently measured. By comparing this simulation with another that used a flow-stress model based on the tension test alone, we found that the equivalent plastic strains differ by less than $5 \%$ at the point of fracture when fracture begins. Apparently when plasticity is localized by sharp notches, the strain at fracture can be determined satisfactorily with a flow-stress model based on the simple tension test. When plasticity is not localized by notches, 


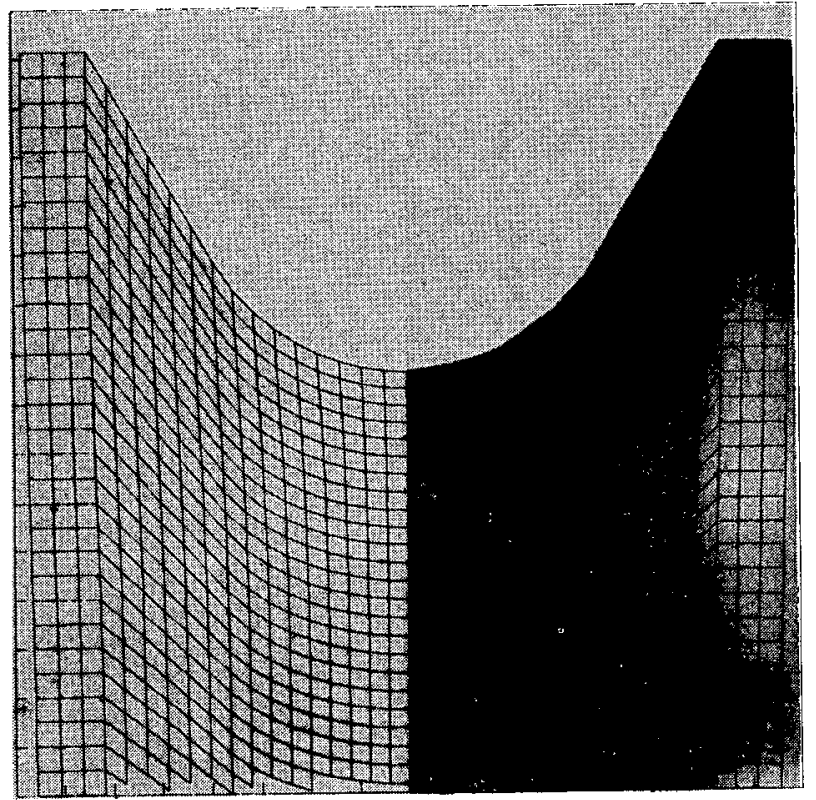

Fig. A.20. Calculational grid and experiment at strain conditions just before fracture of notched aluminum cylinder. Original notch radius $r=0.157$ in. $(4 \mathrm{~mm})$.

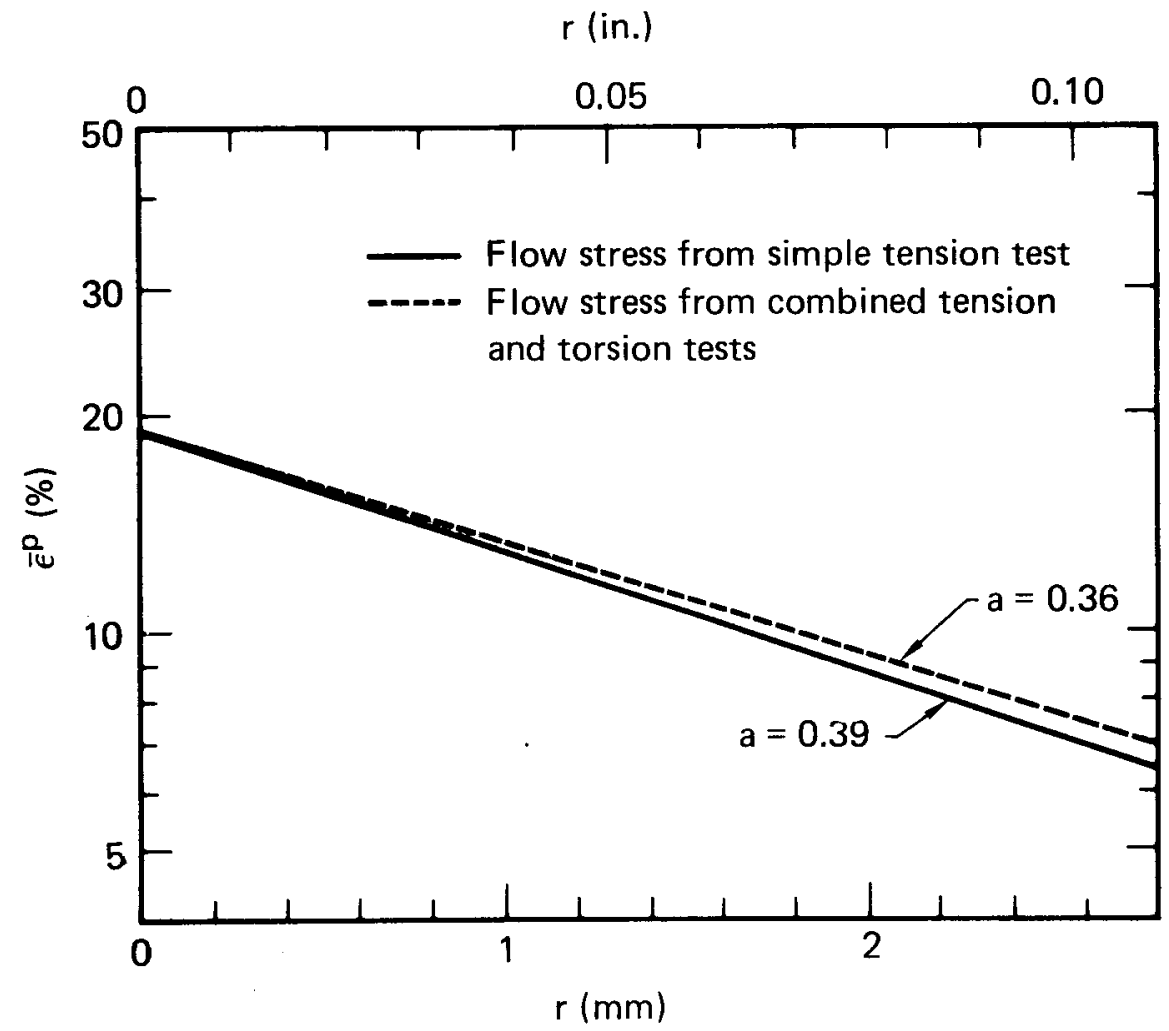

Fig. A.21. Calculated equivalent plastic strain for a notched-cylinder tension test. Notch root radius 0.157 in. $(4 \mathrm{~mm})$. Solid line: $\bar{\varepsilon}^{\mathrm{p}}(\%)=19$ $\exp (-0.39 r)$; dotted line: $\bar{\varepsilon}^{\mathrm{P}}(\%)=19 \exp (-0.36 r)$. 
however, a flow-stress model that includes the effect of stress state is required to obtain the strain at fracture.

This last conclusion is important when one estimates the local equivalent plastic strain for a given overall stretch in a structural member. When we used the flow-stress model with data based on the tension test alone, and matched the stretch across the clip gauge with the stretch in the simulation, we underestimated the equivalent plastic strain at the center of the plate by more than a factor of two. We obtained a much better estimate by using a flow-stress model that included the effect of stress state.

\section{CALIBRATION AND VERIFICATION OF FAILURE CRITERIA IN DUCTILE FRACTURE}

With the constitutive model determined, we can calculate the stress-strain distributions resulting from external loads for various specimen geometries. The objective is to develop a fracture-mechanics methodology for ductile fracture by correlating the observed experimental failure of a specimen with the computer simulation. The calculated stress-strain field at the observed fracture point can be used to develop a model of ductile fracture.

Tension Test of a Plate with a Central Slot. Figure A.22(a) shows the geometry of a centrally, notched tension specimen of 6061-T6 aluminum. Figure A.22(b) shows the calculational grid of the computer model. Because of the symmetry of this geometry, only one-eighth of the physical specimen is required for the model. At the notch root, the Lagrange grid spacing is 0.005 in. $(0.127 \mathrm{~mm})$; hence two zones are used to describe the $0.01-\mathrm{in}$. $(0.254-\mathrm{mm})$ notch-root radius. The grid spacing increases geometrically with distance from the notch. Six zones are used in the half thickness of the plate. The specimen is pulled in tension, perpendicular to the notch, by grips that are 5.5 in. $(14.0 \mathrm{~cm})$ from the notch.

Figure A.23 shows the experimental and calculational values of the load $v s$ the fractional notch opening $\left(h / h^{0}\right)-1$. When correlating calculation with experiment, one should make the physical measurement as close as possible to the region of interest, which in this case is the notch root. For accuracy, a second requirement is the use of an experimentally observable initial condition that undergoes a large change. The change in the notch opening, measured by a clip gage, is used here as a suitable means of matching the experiment and calculation. 

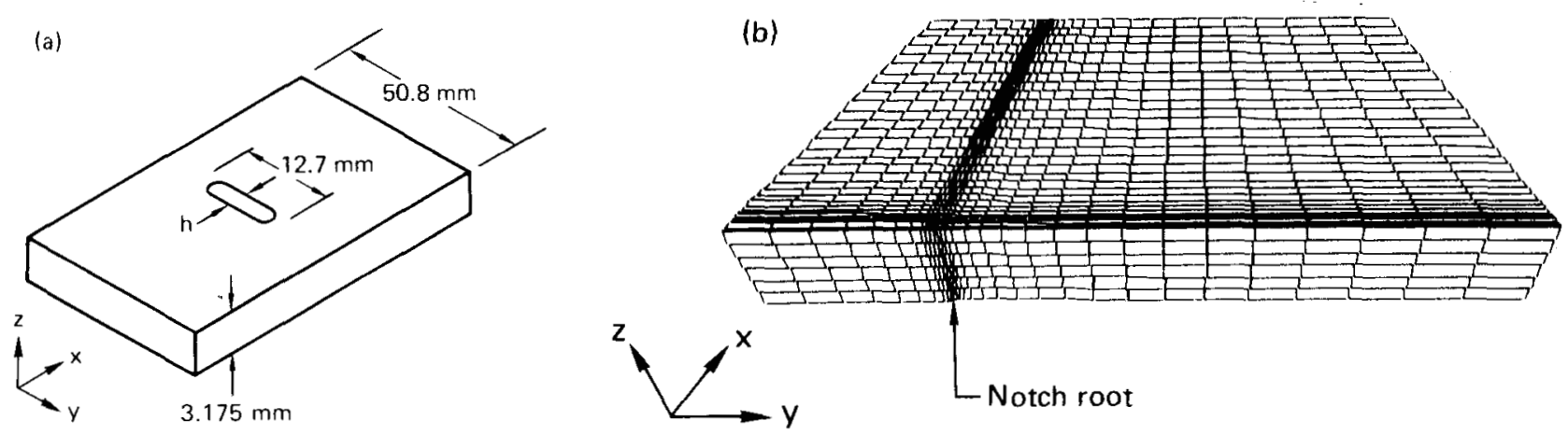

Fig. A.22. Center-notched-plate tension specimen of 6061-T6 aluminum. (a) Geometry, with initial notch width $\mathrm{h}^{0}=0.02 \mathrm{in} .(0.508 \mathrm{~mm})$ and notch-root radius $R=0.01 \mathrm{in}$. $(0.254 \mathrm{~mm})$. (b) HEMP $3 \mathrm{D}$ model of one-eighth of specimen.

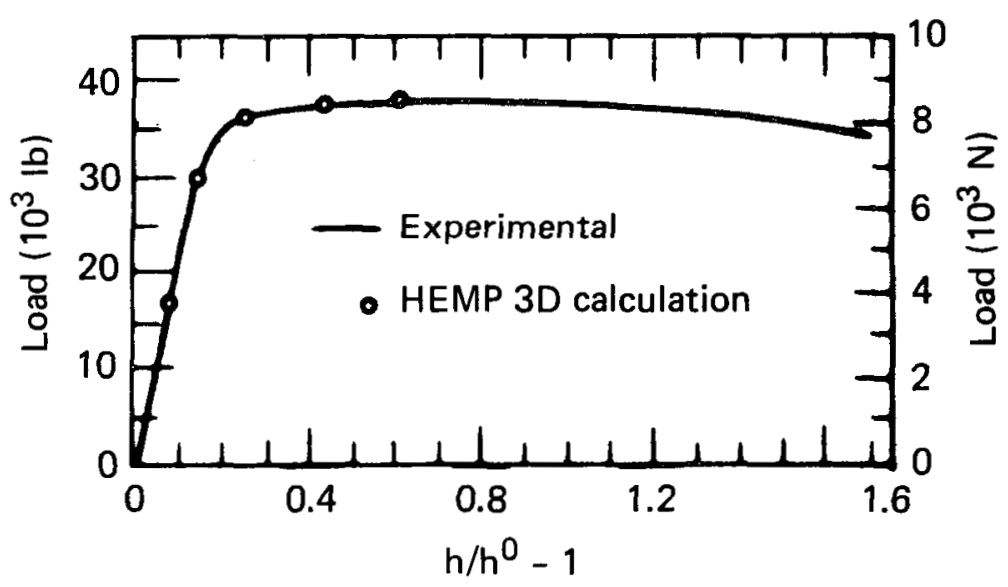

Fig. A.23. Load vs notch opening of center-notched plate pulled in tension.

Figures A.23 and A.24 show that the constitutive model calibrated with the simple tension tests accurately describes all main features of this test. Interrrupted tests showed that flat fracture occurred when $\left(h / h^{0}\right)-1$ was between 0.6 and 0.7 . The flat fracture was a small triangular region at the notch root at the center of the plate as shown in the diagram of Fig A.26. When $\left(h / h^{0}\right)-1$ reached $1.3,45-$ deg shear fracture became visible at the surface.

Figures A.25 and A.26 show the calculated stress and strain distribution at the notch tip just before the experimental flat fracture occurs. Figure A.26 includes a sketch of the experimentally observed flat fracture region, which appears as a triangle with a base along the notch root. The base extends \pm 0.039 in. $( \pm 1 \mathrm{~mm})$ in the thickness direction from the plane of symmetry. 


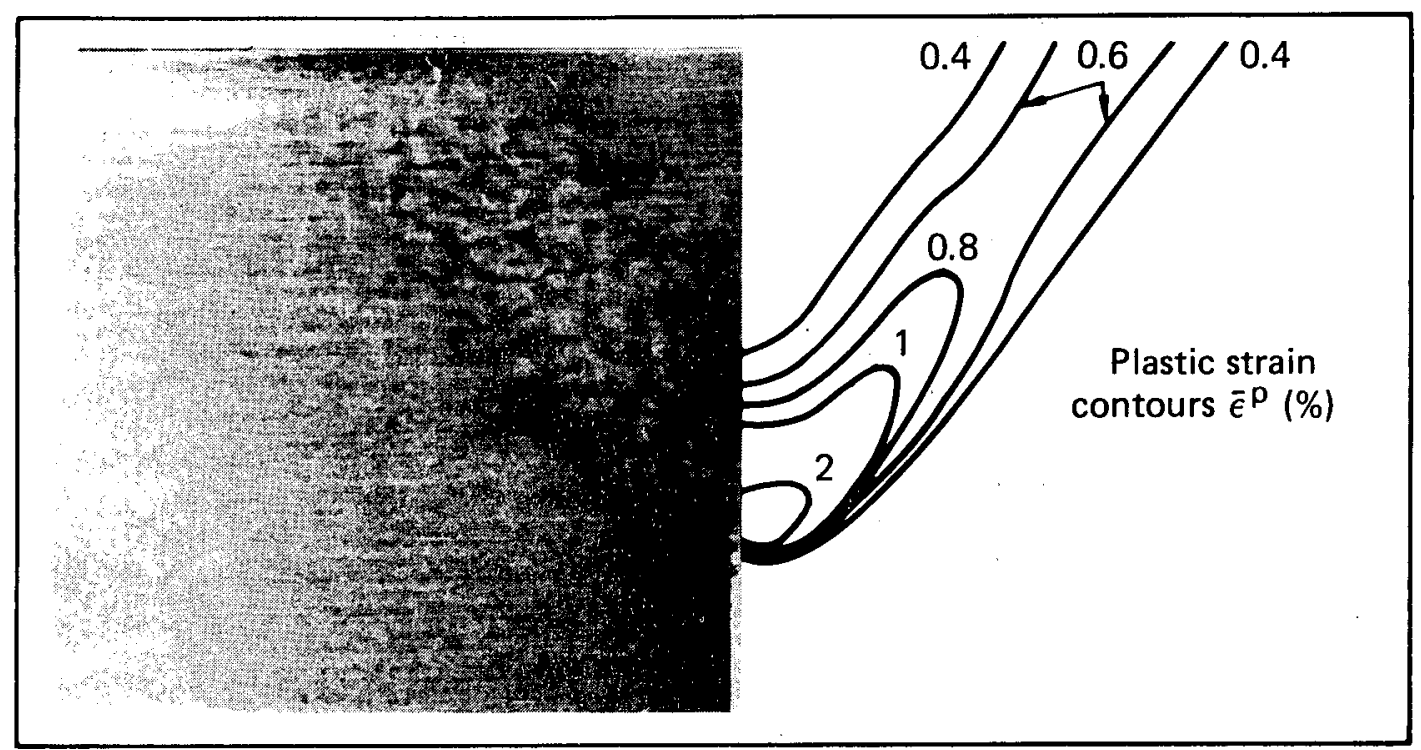

Fig. A.24. Experimental (left) and calculated (right) plastic-flow field of the centrally notched plate when $\left(h / h^{0}\right)-1=0.6$. The calculations show contours of constant equivalent plastic strain.

(in.)

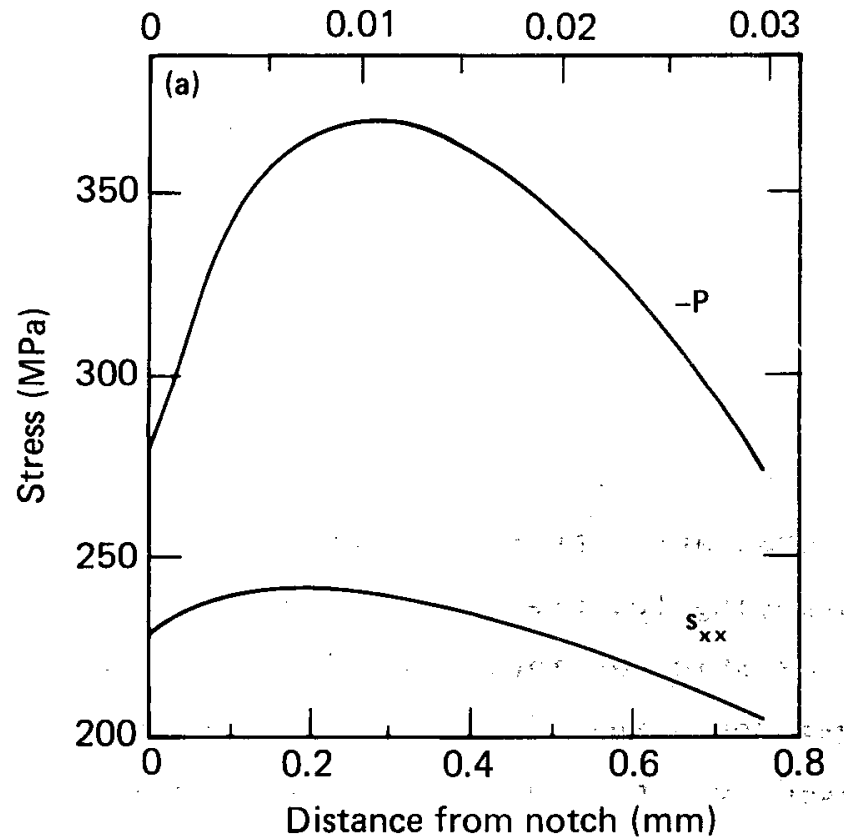

(in.)

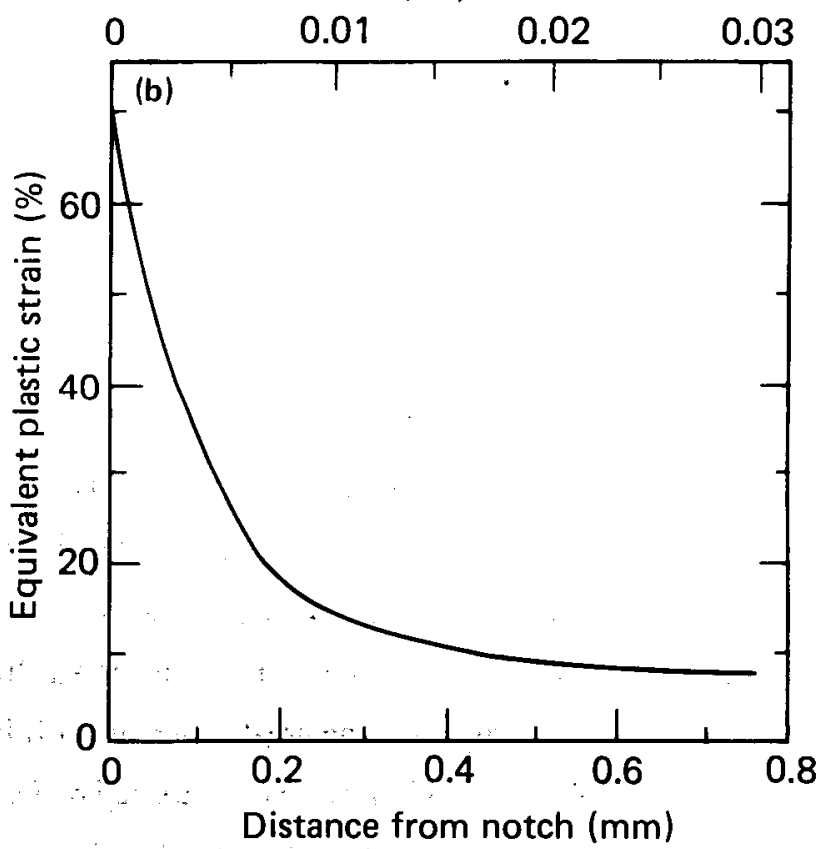

Fig. A.25. Calculated profiles of stress and strain at fracture initiation, $\left(\mathrm{h} / \mathrm{h}^{0}\right)-1=0.6$, vs distance from notch of a center-notched plate at mid-thickness. (a) Hydrostatic stress $-\mathrm{P}$ and stress deviator $\mathbf{s}_{\mathbf{x x}}$ in the direction of tension. (b) Equivalent plastic strain. 


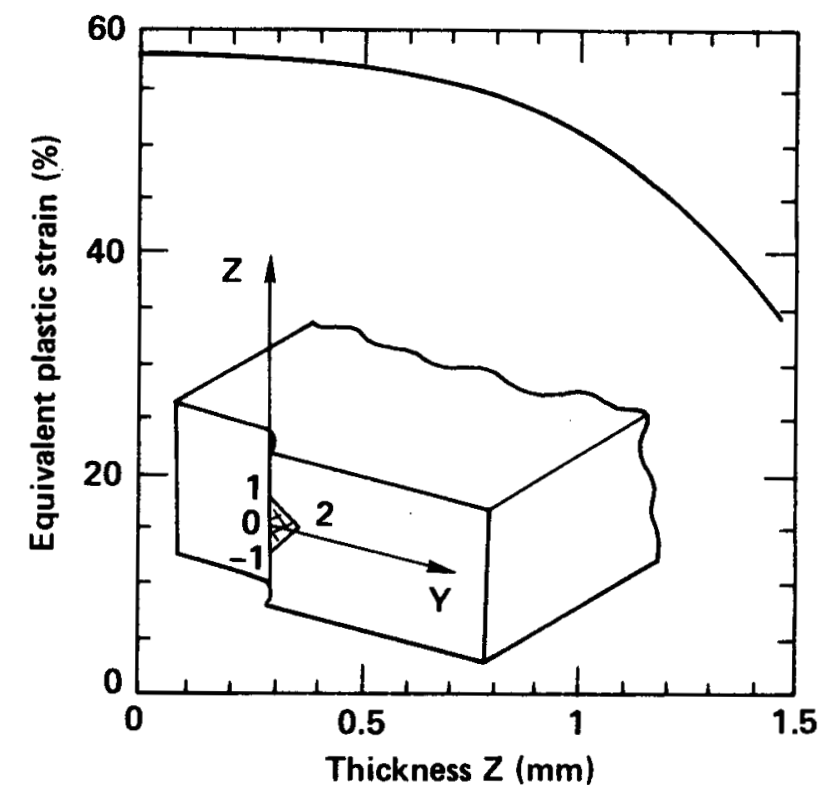

Fig. A.26. Calculated equivalent plastic-strain profile through the thickness at a position 0.001 in. $(0.025$ $\mathrm{mm}$ ) ahead of the notch, when $\left(\mathrm{h} / \mathrm{h}^{0}\right)-1=0.6$. The sketch shows the observed triangular region of flat fracture.

The calculation shows that the equivalent plastic strain decreases beyond the 0.039-in. (1-mm) position (Fig. A.26). This is consistent with the fracture of the simple tension test, which occurred at an equivalent plastic strain between 0.5 and 0.6 .

\section{Notched-Tension Tests}

The simple tension test was used to establish the critical damage under near-uniform strain and relatively low hydrostatic stress. Notched-tension tests provide an experimental means of studying failure under conditions of large localized strains and enhanced hydrostatic tension. Changing the notch sharpness or the notch-root radius changes the strain field in the specimen at failure. The localized high strain is used to determine the critical volume or size dimension over which strain accumulation must be evaluated; high hydrostatic tension can be related to void-growth models of ductile fracture. High hydrostatic tension is similar to the stress state at the tip of a sharp crack in plane strain as employed in $K_{I c}$ fracture testing. Four sets of geometrically similar specimens are defined in Table A.1. In all, thirteen different specimen geometries were tested. 
Experimental Evaluation. Specimens were loaded in a servo-hydraulic test machine under stroke control. Plots of load vs stroke were made during the test, and a very accurate strain measurement was made to permit the computer simulations to be correlated with the experiment.

Two factors were considered in choosing a means to match the experiments with the calculations. First, the correlation should be made in the region of

TABLE A. 1

\begin{tabular}{|c|c|c|c|c|c|c|}
\hline Geometry & Size & $D(\mathrm{~mm})$ & $\mathrm{d}(\mathrm{mm})$ & $R(\mathrm{~mm})$ & $\bar{\varepsilon}_{\mathrm{N}}^{\mathrm{p}}(\%)$ & $a(\mathrm{~mm})^{-1}$ \\
\hline \multirow[t]{4}{*}{ I } & A & 12.7 & 9.50 & 1.0 & 30 & 1.56 \\
\hline & B & 25.4 & 19.1 & 2.0 & 23 & 0.78 \\
\hline & C & 50.8 & 38.1 & 4.1 & 20 & 0.39 \\
\hline & D & 76.2 & 57.2 & 6.1 & 19 & 0.26 \\
\hline \multirow[t]{4}{*}{ II } & $\mathrm{A}$ & 12.7 & 9.50 & 0.51 & 33 & 3.28 \\
\hline & B & 25.4 & 19.1 & 1.0 & 25 & 1.64 \\
\hline & C & 50.8 & 38.1 & 2.0 & 23 & 0.82 \\
\hline & D & 76.2 & 57.2 & 3.0 & 22 & 0.55 \\
\hline \multirow[t]{3}{*}{ I I I } & B & 25.4 & 19.1 & 0.51 & 35 & 3.33 \\
\hline & $\mathrm{C}$ & 50.8 & 38.1 & 1.0 & 25 & 1.67 \\
\hline & D & 76.2 & 57.2 & 1.5 & 21 & 1.11. \\
\hline \multirow[t]{2}{*}{ IV } & C & 50.8 & 38.1 & 0.51 & 43 & 3.33 \\
\hline & D & 76.2 & 57.2 & 0.76 & 38 & 2.22 \\
\hline
\end{tabular}

${ }^{*} \bar{\varepsilon}_{N}=\begin{aligned} & \text { calculated equivalent plastic strain at the notch when fracture } \\ & \text { occurs: }\end{aligned}$ $\bar{\varepsilon}^{\mathrm{P}}=\bar{\varepsilon}_{\mathrm{N}}^{\mathrm{P}} \mathrm{e}^{-\mathrm{ar}}$,

where $r$ is the distance from the notch in the radius direction and $a$ is the strain field decay constant.

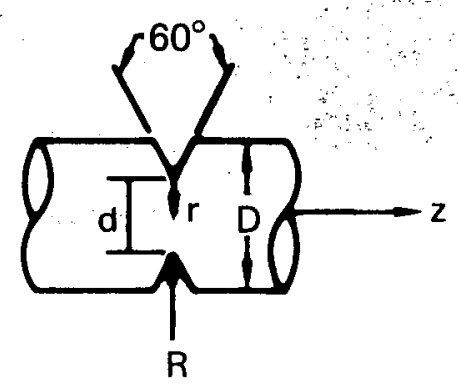


interest; in this case, the region of interest is the notch root, at which fracture begins. Second, to improve data sensitivity, the strain should be measured on a dimension that undergoes a large change. A photographic technique was used to monitor notch-geometry changes. Photographs were correlated to the loading curve by electronic marking on the load-stroke plot. In this way, the notch geometry at the point of incipient fracture, as determined from experimental photographs, can be compared directly with the computer-predicted notch geometries. The calculations produce a time sequence of fields corresponding to increasing displacement. The calculated stress/strain field at fracture initiation was selected by choosing the time at which the notch geometry in the simulation most closely resembled the photograph immediately before failure. Figure A.27 shows an overlay of the experimental notch geometries before loading and just before failure on the corresponding computer simulation.

For very small notch radii, the photographic method was indadequate because we could not accurately observe changes in notch geometry. With these specimens (experiments $\mathrm{A}$ and $\mathrm{B}$ in Table A.1), we supplemented the photographic procedure by spanning the notch with a $0.50-\mathrm{in} .(12.7-\mathrm{mm})$ extensometer. Although this method does not make use of the large geometry change at the root of the notch, the sensitivity of the extensometer gave enough accuracy that we could match a measurement with the computer simulation. In this case, we determined the computer time of fracture onset by matching the elongation
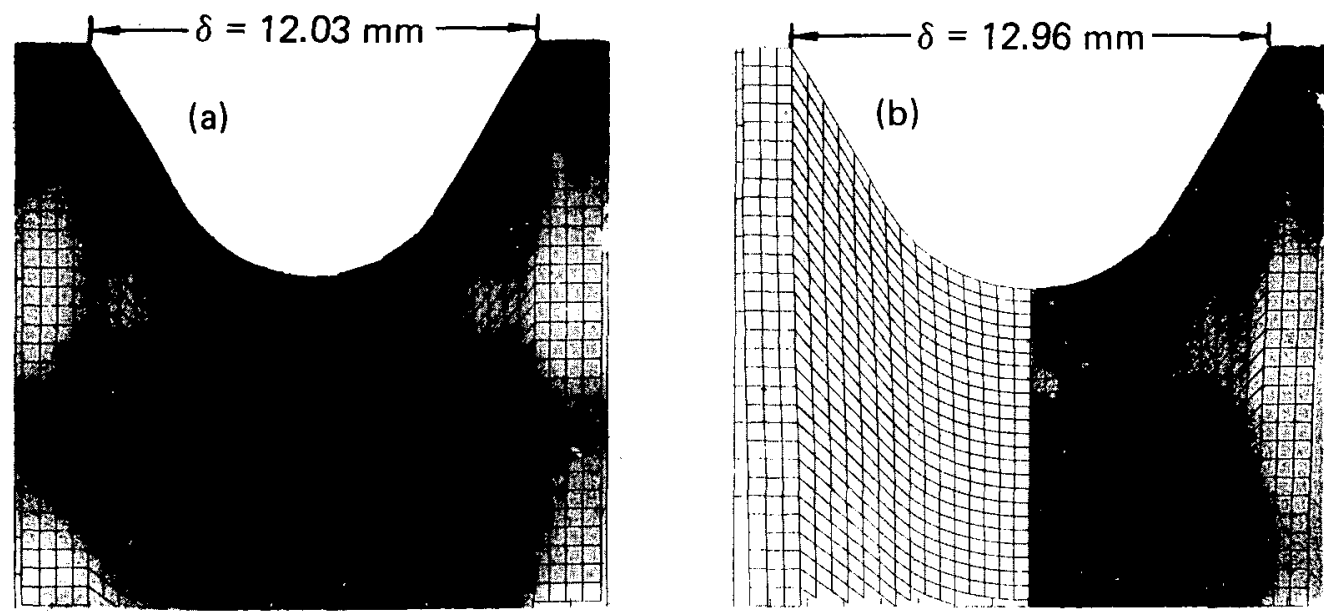

Fig. A.27. Notched tension specimen geometry I C. (a) Calculational grid overlayed on experiment at initial conditions. (b) Calculational grid and experiment at strain conditions just before fracture. 
across the notch and the extensometer reading at failure. When the photographic technique yielded valid results, the two methods agreed very well.

Calculations. Figure A.28 shows stress conditions near the time of failure for geometry III $B$, in which the root radius is $0.020 \mathrm{in.}(0.50 \mathrm{~mm})$. A1l the specimens shown in Table 1 gave similar stress patterns. The peak stress occurs slightly below the notch, and the hydrostatic pressure at the notch is about $29 \mathrm{ksi}\left(200 \mathrm{MPa}\right.$ ) tension. The ratio $\mathrm{s}_{2} / \mathrm{s}_{3}$ is about 0.1 at the notch and rises rapidly to 1 slightly below the surface.

Figure A.29 shows the equivalent plastic strain field for geometry II B at three different load extensions. The parameter $\Delta \mathrm{L}$ refers to the displacement of a point on the axis originally $2.756 \mathrm{in}$. (70 mm) from the center. The three lines were obtained by plotting the calculated equivalent plastic strain for each zone of the calculational grid. The figure shows that the equivalent plastic strain has the form $\bar{\varepsilon}^{\mathrm{P}}=\bar{\varepsilon}_{\mathrm{N}}^{\mathrm{P}} \exp (-a r)$, where $\bar{\varepsilon}_{\mathrm{N}}^{\mathrm{P}}$ is the strain at the notch and $r$ the distance below the notch. The decay constant a was found to be the same as the load extension increased, as shown in Fig. A.29. All the other geometries gave similar results, so that for any
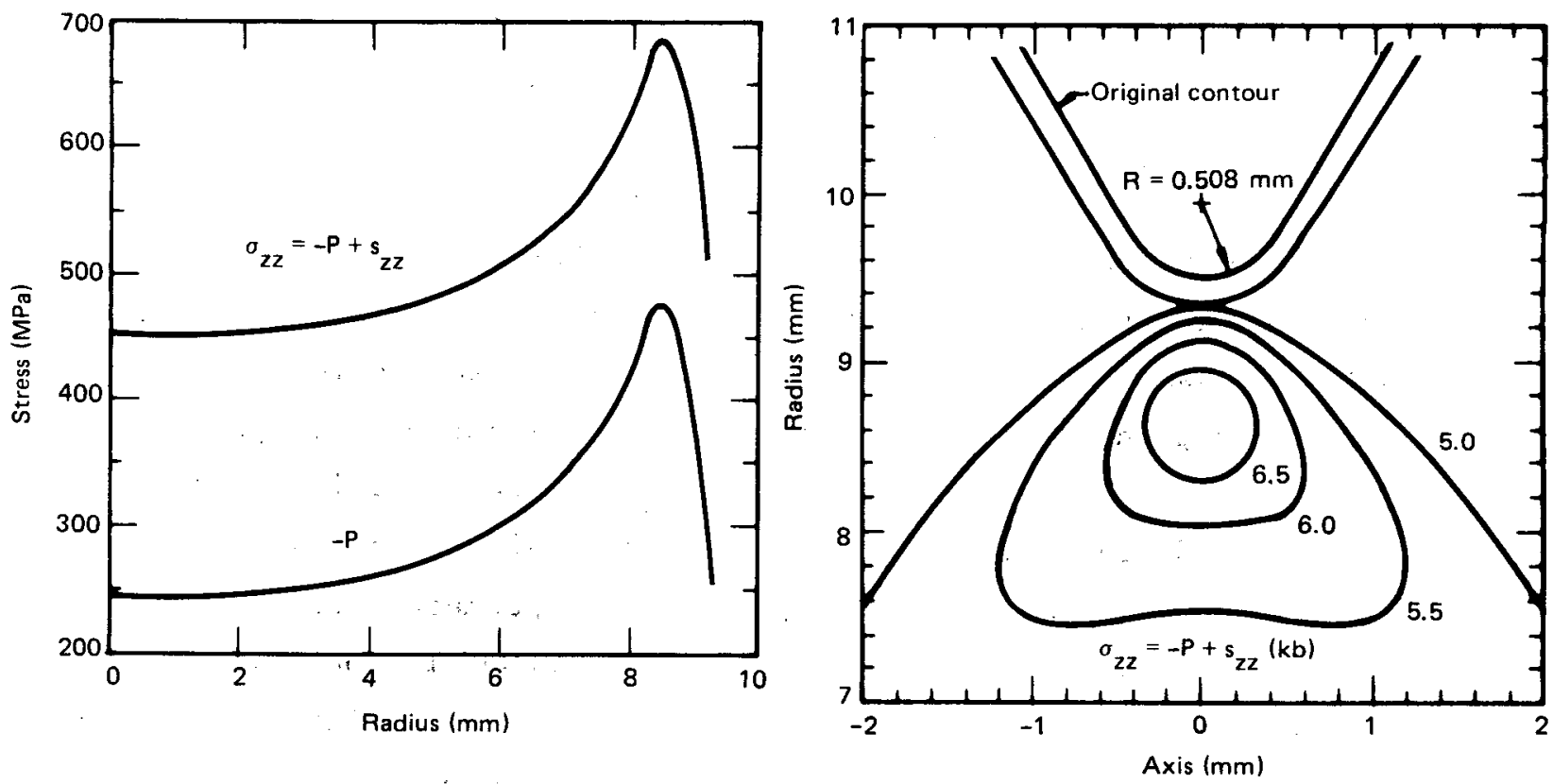

Fig. A.28. Calculated stress fields for geometry III B. (a) Axial stress $\sigma_{z z}$ and hydrostatic pressure $p$ at midplane vs radius. (b) Contours of axial stress at notch tip. 


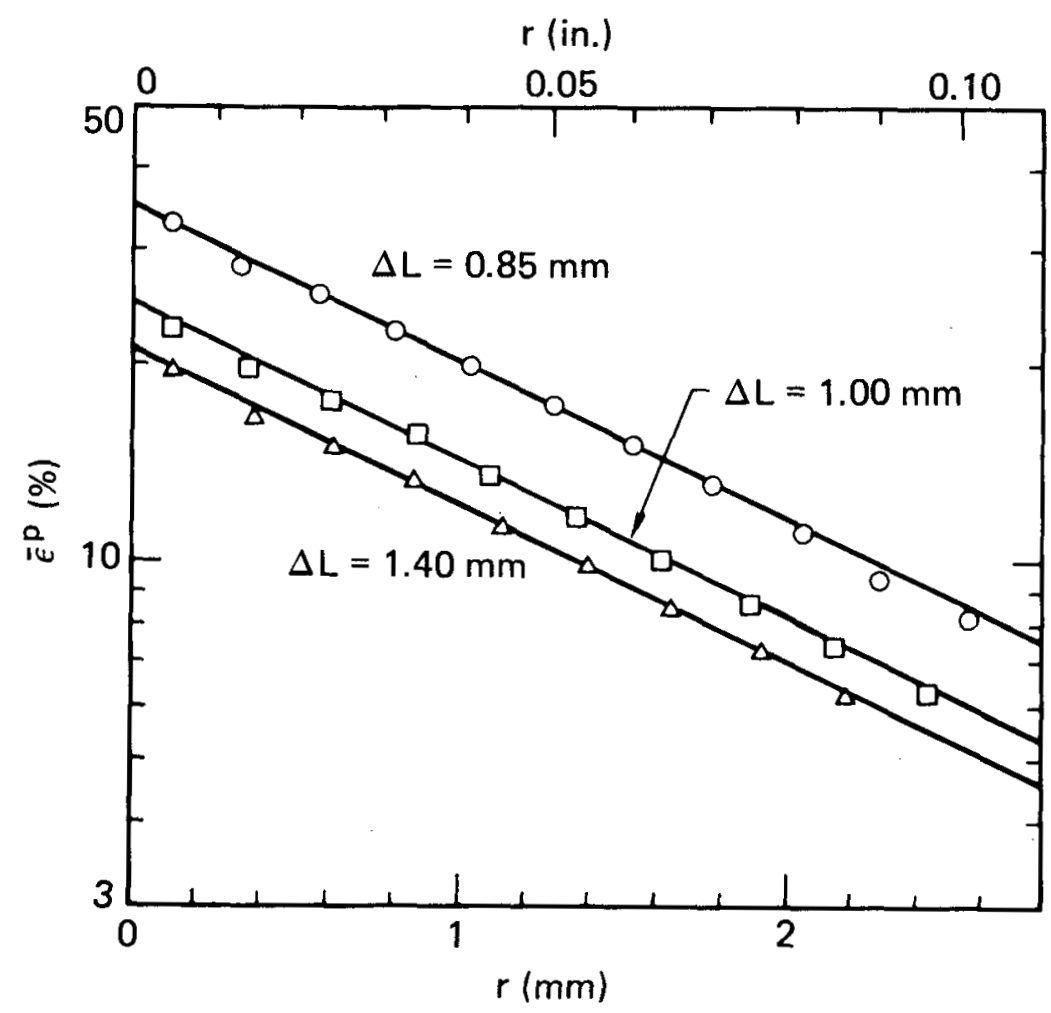

Fig A.29. Equivalent plastic strain vs distance from notch for different extension $\Delta \mathrm{L}$ of geometry II D.

one geometry the product $a R$ of the decay constant and root radius is constant. Moreover, the product aR has very nearly the same value for all the geometries: This shows that the notch establishes the local strain field irrespective of other dimensions. Table 1 gives the decay constant $a$ and the equivalent plastic strain at the notch at fracture for all the specimens. Figure A.30(a) shows the calculated strain field at fracture for geometry I experiments. The point of intersection of the lines can be interpreted as a critical strain of $18 \%$ and a critical distance of about $0.01 \mathrm{in} .(0.3 \mathrm{~mm})$. Similarly, Fig. A.30(b) gives a critical strain of $20 \%$ and a critical distance of about $0.006 \mathrm{in.}(0.15 \mathrm{~mm})$ for geometry II. For geometry III, Fig. A.30(c) shows a critical strain of $18 \%$ and a critical distance of about 0.008 in. $(0.2 \mathrm{~mm})$. The uncertainty in establishing the correct correlation time for the calculation gives an error in $\bar{\varepsilon}^{\mathrm{P}}$ of about $\pm 10 \%$. Hence no conclusions can be drawn for the results of geometry IV shown in Fig. A.30(d), since the experiments correlated to computer simulations gave the same result within the uncertainty. Actually, to establish a critical strain and distance by this 

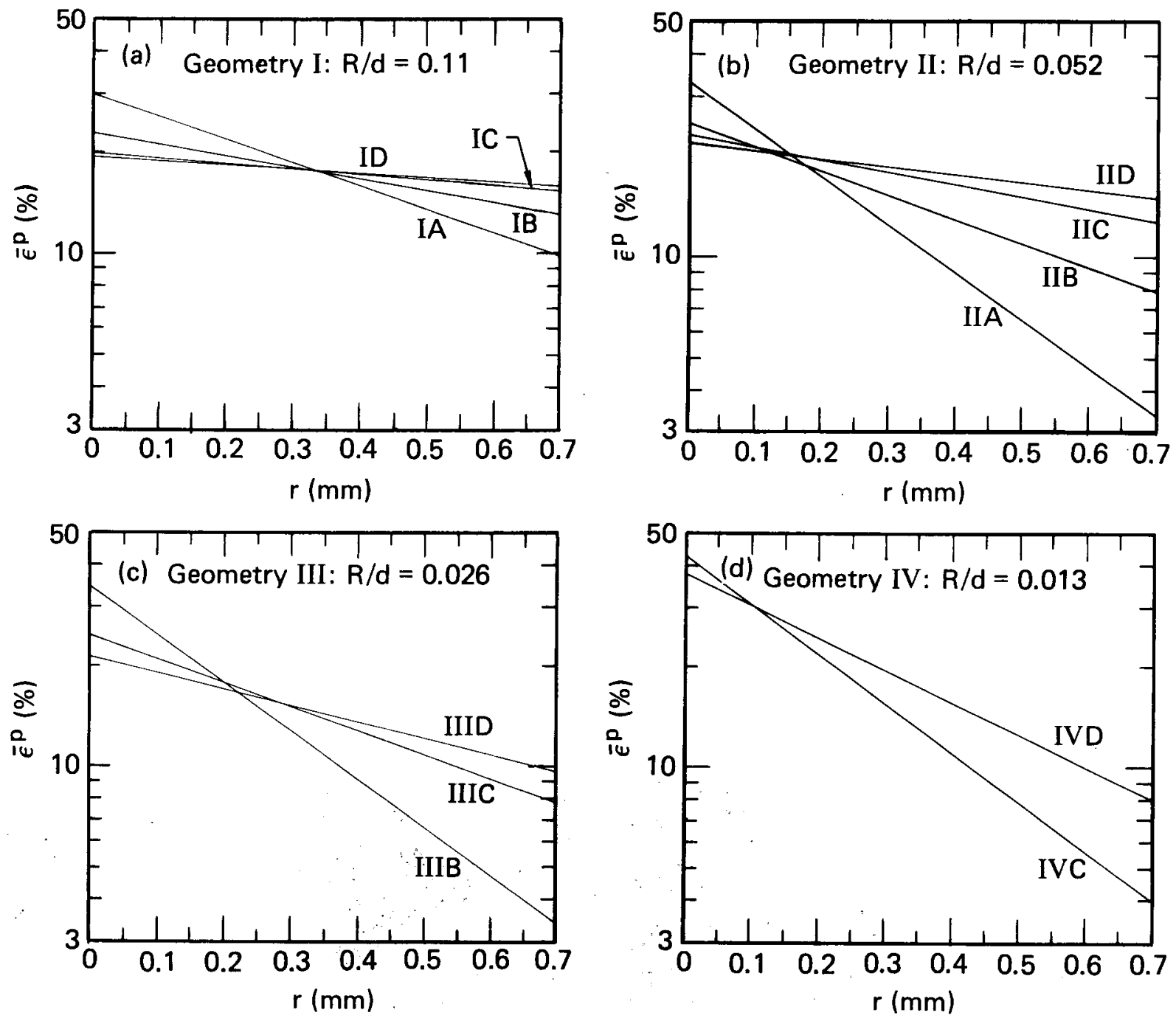

Fig. A.30. Equivalent plastic strain vs distance from the notch at time of fracture for four ratios of root radius to diameter.

method requires notch radii about as large as the critical distance. Thus, the scaled experiment should include notches of about $0.01-i n \cdot(0.3-\mathrm{mm})$ root radius.

\section{Torsion Tests}

In the previous section we discussed the results of torsion tests on solid 6061-T651 aluminum with reference to the evaluation of the material flow model. These tests were also used to assess the critical damage, i.e., the equivalent plastic strain, required for shear fracture under conditions of large rotation. 
Figure A.10 compares the specimen and a computer simulation of the test at imminent failure. The final shear angle is measured along the centerline of the specimen to avoid parallax errors arising from the curvature of the specimen surface away from the plane of the photograph. The localized shear twist angle $\phi$ measured along the centerline of the reduced section was $67 \pm 2$ deg. The idea that this critical shear twist angle is a constant was subsequently confirmed on specimens of radii from $0.188 \mathrm{in.}(4.76 \mathrm{~mm}$ ) to 0.375 in. $(9.53 \mathrm{~mm})$. Figure A.31 shows the specimen surface at failure. The equivalent plastic strain at fracture is $100 \%$. This torsion result shows an increase in ductility over the tension tests, in which the equivalent plastic strain at failure was about $60 \%$. The torsion test has a zero hydrostatic stress component. This will suppress void growth due to hydrostatic tension. An increased deformation would result for the shear component acting alone, because the inherent voids are relatively small and widely spaced.

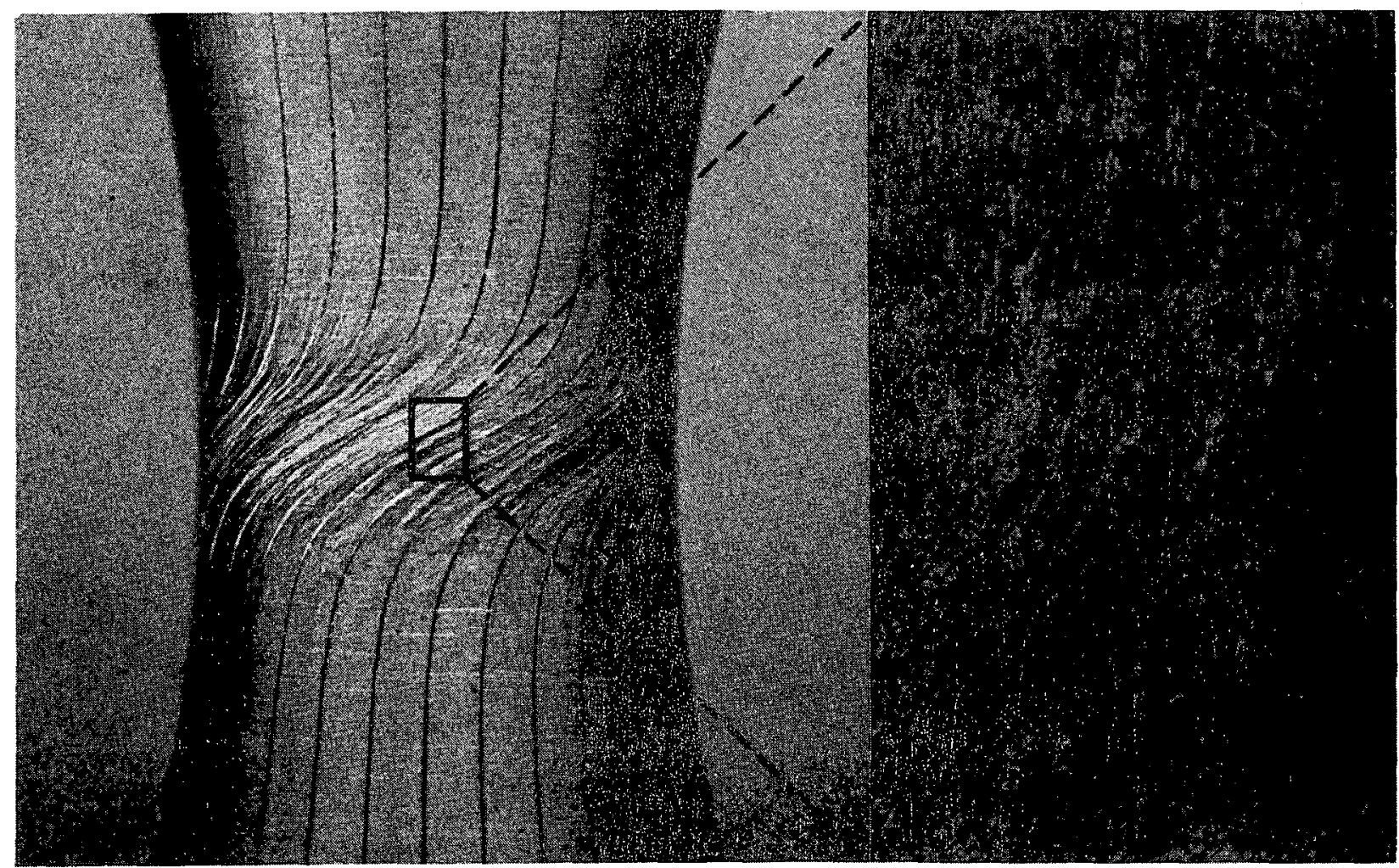

Fig. A.31. Surface of 0.75-in. (19.05-mm) diam solid torsion specimen of 6061-T651 aluminum at failure. The right side shows the grain microstructure 0.025 in. $(0.635 \mathrm{~mm})$ below the surface. 
Tension-Torsion Tests

Results discussed in the previous sections related to the application of an appropriate constitutive model and critical damage factor to specimens subjected to a single load system, i.e., tension or torsion. The relation between hydrostatic tension and shear due to combined load systems can be associated with the void-growth-and-coalescence mechanism of ductile fracture. In this respect, both the sequence and the magnitude of applied loads should influence the critical damage requirement for ductile fracture. To investigate this, we tested a series of tension-torsion samples ranging in minimum diameter from $0.377 \mathrm{in.}(9.58 \mathrm{~mm})$ to $0.751 \mathrm{in.}(19.08 \mathrm{~mm})$. As with the torsion samples, the section diameter increased away from the specimen center.

Four samples were deformed in tension up to a prescribed radial strain, and then twisted to failure; we observed the final shear strain angle. Figure A.32 shows typical results. The early stages of ductile fracture relate to void growth due to hydrostatic tension. The initial tension should therefore open voids in the reduced section, and the subsequent torsion should enhance the shear 1 ink-up of these voids. It was anticipated that the resulting failure strain would be less than that resulting from torsion only, because of the mechanisms of enhanced void growth and coalesence. The results, however, indicated only a minor reduction in strain. The localization of shear strain was generally confined to the region of high tensile deformation.

The magnitude of the shear strain to failure in the combined-load test can be understood by considering the location of damage accumulation in the tension and torsion phases of the test. During tensile loading, the highest hydrostatic stresses are at the specimen center (see Fig. A.5), and drop rapidly near the outside radius. Thus it is in this central region that the void-growth mechanism is active." During the torsion phase of the loading, on the other hand, the maximum shear (and thus the void-coalesence mechanism) is acting on the outer fibers. Since the two load systems are essentially acting on different material, the effect of the load sequence is not realized. A thin-walled cylinder, in which void growth due to tension and shear due to torsion act on the same mater.al, should exhibit a more pronounced effect. 


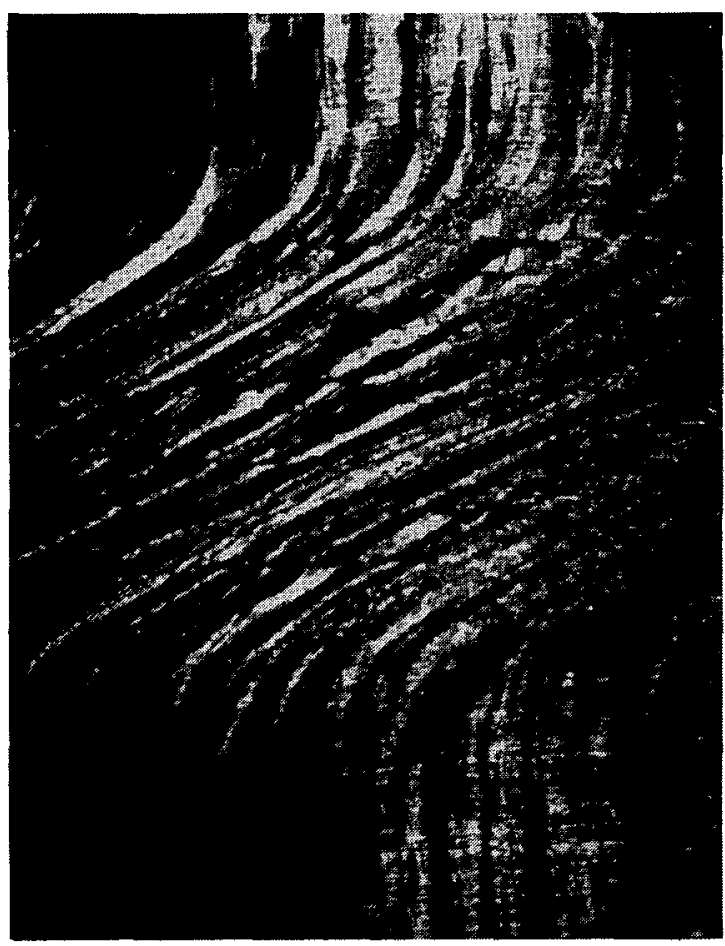

Fig. A.32. Shear deformation and cracking on the surface of a tension-torsion sample. Specimen was loaded to an axial strain $\bar{\varepsilon}$ p $=0.20$ and then twisted until cracking was observed.

\section{Conclusion}

Notched-tension tests offer a possiblity of determining a fracture-size effect with relatively small test specimens. For accuracy, the experiments should be conducted with notch radii near the dimension of the expected size effect. A major difficulty is the correlation of the computer simulation with the experiment; this difficulty arises because of the relatively small displacement in notched-tension tests. The fracture strain for 6061-T651 is about half the value estimated for 6061-T6 aluminum. 


\section{REFERENCES}

1. G. C. Sih, Properties Related to Fracture Toughness (ASTM STP 605) (American Society for Testing and Materials, Philadelphia, 1976).

2. G. R. Irwin and P. C. Paris, "Fundamental Aspects of Crack Growth and Fracture," in Fracture, an Advanced Treatise, H. Liebowitz, Ed.

(Acadamic Press, New York-London, 1971) Vol 3.

3. J. E. Srawley and W. F. Brown, Jr, Fracture Toughness Testing and Its Applications (ASTM STP 381), (American Society for Testing and Materials, Philadelphia, 1965), pp. 133-198.

4. G. R. Irwin, "Fracture Mode Transition for a Crack Traversing a Plate," Trans. ASME 82D (2), 417-425, (June, 1960).

5. V. M. Vainshelbaum and R. V. Goldstein, "On the Material Length Scale as a Measure of the Fracture Toughness of Plastic Materials, and Its Role in Fracture Mechanics," Institute of Problems of Mechanics, USSR Academy of Science, Moscow, Preprint No. 77 (1976).

6. W. F. Brown, Jr., and J. E. Srawley, Plane Strain Crack Toughness Testing of High Strength Metallic Materials (ASTM STP 410) (American Society for Testing and Materials, Philadelphia, 1966).

7. J. R. Rice, "A Path Independent Integral and Approximate Analys is of Strain Concentration by Notches and Cracks," Trans. ASME (J. Appl. Mech.) 35E, 379-386 (June, 1968).

8. J. W. Hutchinson, "Singular Behavior of the End of a Pencil Crack in a Hardening Material," J. Mech. Phys. Solids 16, 13-31 (1968).

9. J. R. Rice and G. F. Rosengren, "Plane Strain Deformation Near a Crack Tip in a Power-Law Hardening Material," J. Mech. Phys. Solids 16 1-12, (1968).

10. F. A. McClintock, in Fracture, an Advanced Treatise, H. Liebowitz, Ed. (Acadamic Press, New York-London, 1971) Vo1 3, pp. 47-225.

11. J. A. Begley and J. D. "Landes, "The J Integral as a Fracture Criterion," in Fracture Toughness (ASTM STP 514) (American Society for Testing and Materia1s, Philadelphia, 1971), pp. 1-23. 
12. J. D. Landes and J. A. Begley, "Test Results from J-Integral Studies, An Attempt to Establish a J Ic Test Procedure," in Fracture Analysis (ASTM STP 560) (American Society for Testing and Materials, Philadelphia, 1974), Pp. 170-186.

13. C. F. Shih, H. G. De Lorenzi, and W. R. Andrews, Studies on Crack Initiation and Stable Crack Growth (ASTM STP 668) (American Society for Testing and Materials, Philadelphia, 1976).

14. Marston, T. and Jones, R., "R and D Status Report, Nuclear Power Division," EPRI Journal 4 (No. 9), 45 (November, 1979).

15. F. R. Tuler and B. M. Butcher, "A criterion for the Time Dependence of Dynamic Fracture," Int. J. Fracture Mechanics 4 (No. 4), 431 (1968).

16. F. A. McClintock, "Plasticity Aspects of Fracture," in Fracture, an Advanced Treatise, H. Liebowitz, Ed. (Acadamic Press, New York-London, 1971) Vo1 3.

17. K. Mogi, "Rock Fracture," in Annual Review of Earth and Planetary Science, F. A. Donath, ed. (Annual Reviews, Palo Alto, CA, 1973), Vol. 1, pp. 63-84.

18. J. A. Begley, W. A. Logsdon, and J. D. Landes, Ductile Rupture Blunt Notch Fracture Criterion--Flaw Growth and Fracture (ASTM STP 631) (American Society for Testing and Materials, Philadelphia, 1977).

19. M. L. Wilkins, R. E. Blum, E. Cronshagen, and P. Grantham, A Method:for Computer Simulation of Problems in Solid Mechanics and Gas Dynamics in Three Dimensions and Time, Lawrence Livermore National Laboratory, Livermore, CA, UCRL-51574, Rev. I (1975).

20. D. M. Norris, Jr., J. E. Reaugh, B. Moran, and D. F. Quinones, "Computer Model for Ductile Fracture: Applications to the Charpy V-Notch Test," Electric Power Research Institute, Palo Alto, CA, EPRI NP-961 (January 1979).

21. R. M. McMeeking and D. M. Parks, "On Criteria for J-Dominance of Crack-Tip Fields in Large Scale Yielding," ASTM E-24 Symposium on Elastic-Plastic Fracture, Atlanta, GA, November 1977.

22. M. L. Wilkins, Calculation of Elastic-Plastic Flow, Lawrence Livermore National Laboratory, Livermore, CA, UCRL-7322 Rev. 1 (1959).

23. D. C. Drucker, "A Definition of Stable Inelastic Material," J. Appl. Mech. 26, 106 (No. 101, 1959). 
24. R. Hill, The Mathematical Theory of Plasticity (Oxford Univ. Press, Oxford, 1971) Chapters 2, 3.

25. D. R. Hayhurst and B. Storakers, "Creep Rupture of the Andrade Shear Disk", Proc. R. SoC. London A 349, 369-382 (1976). 